\title{
Accessing Pentagonal Bipyramidal Geometry with Pentadentate Pincer Amido-bis(amidate) Ligands in Group IV and V Early Transition Metal Complexes
}

Orhi Esarte Palomero, Richard A. Jones

The University of Texas at Austin - Department of Chemistry

Supporting Information 


\section{Table of contents}

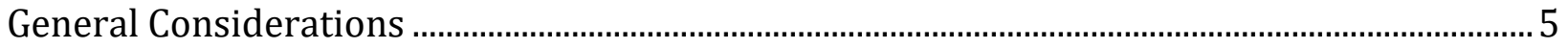

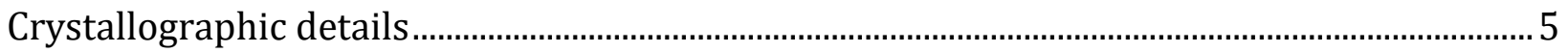

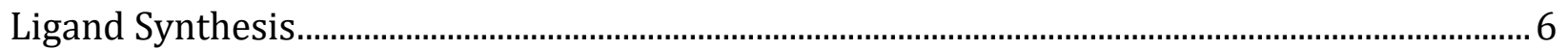

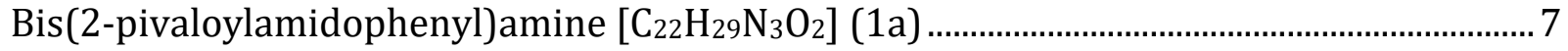

Bis(2-mesitoylamidophenyl)amine $\left[\mathrm{C}_{32} \mathrm{H}_{33} \mathrm{~N}_{3} \mathrm{O}_{2}\right](1 \mathrm{~b})$................................................... 8

Bis(2-adamantoylamidophenyl)amine $\left[\mathrm{C}_{34} \mathrm{H}_{41} \mathrm{~N}_{3} \mathrm{O}_{2}\right]$ (1c) ……............................................

Bis(2,4,6-(tris(Isopropyl)benzoylphenyl))amine [ $\mathrm{C}_{44} \mathrm{H}_{57} \mathrm{~N}_{3} \mathrm{O}_{2}$ ] (1d).................................... 10

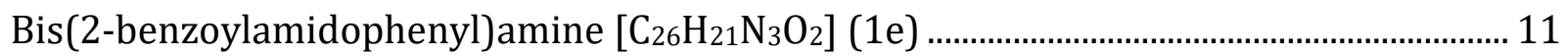

Bis(2-((N,N-dimethyl)benzoylamidine)phenyl)amine [ $\left.\mathrm{C}_{30} \mathrm{H}_{31} \mathrm{~N}_{5}\right]$ (8) .............................. 12

Metal Complex Synthesis ...................................................................................................... 13

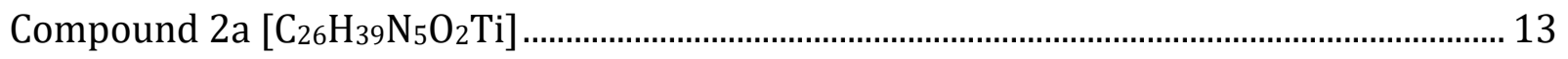

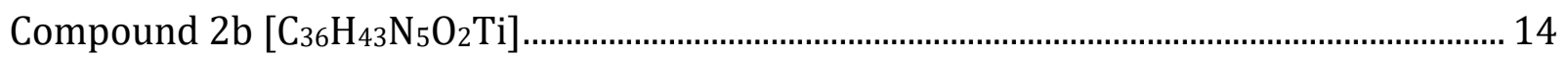

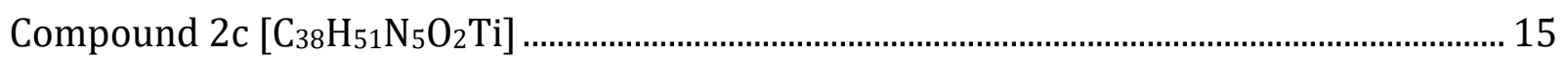

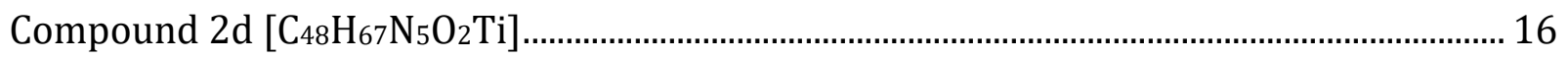

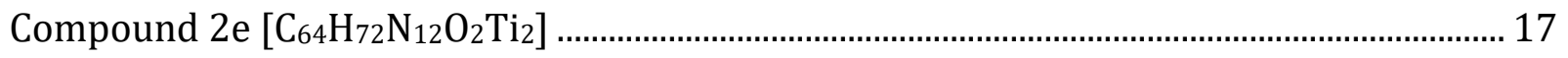

Compound 3d [ $\left.\mathrm{C}_{48} \mathrm{H}_{67} \mathrm{~N}_{5} \mathrm{O}_{2} \mathrm{Zr}\right]$..................................................................................... 18

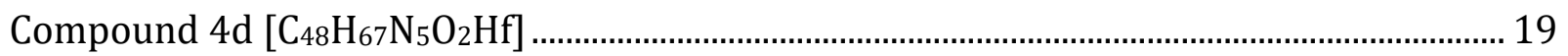

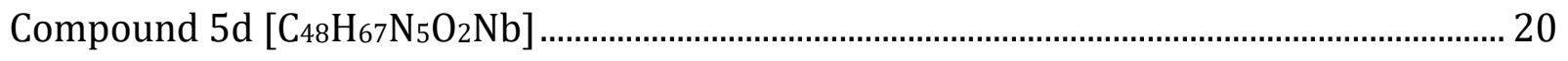

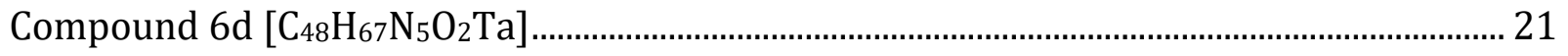

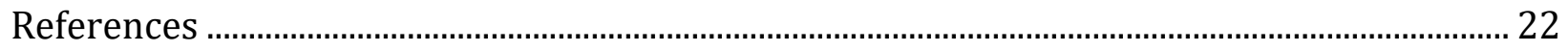

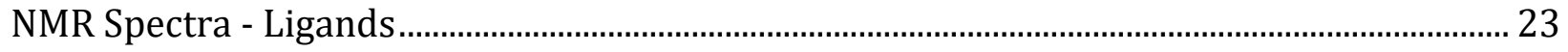

NMR Spectra - Metal Complexes ........................................................................................................ 42

\section{Table of figures}

Figure S 1 Solid-state packing of ligand 1a with hydrogen bonding interactions (dash lines). Non hydrogen bonding hydrogens removed for clarity. ............................................................... 6

Figure $\mathrm{S} 2{ }^{1} \mathrm{H}-\mathrm{NMR}$ spectrum of $1 \mathrm{a}$ in $\mathrm{CDCl}_{3}$ at room temperature ......................................... 23

Figure $\mathrm{S} 3{ }^{13} \mathrm{C}$-NMR spectrum of $1 \mathrm{a}$ in $\mathrm{CDCl}_{3}$ at room temperature ....................................... 24

Figure S $4 \mathrm{HSQC}$ spectrum of $1 \mathrm{a}$ in $\mathrm{CDCl}_{3}$ at room temperature …………….......................... 25

Figure $\mathrm{S} 5^{1} \mathrm{H}-\mathrm{NMR}$ spectrum of $\mathrm{1b}$ in $\mathrm{CDCl}_{3}$ at room temperature......................................... 26

Figure $\mathrm{S} 6{ }^{13} \mathrm{C}$-NMR spectrum of $\mathrm{1b}$ in $\mathrm{CDCl}_{3}$ at room temperature......................................... 27

Figure S 7 HSQC-NMR spectrum of $1 \mathrm{~b}$ in $\mathrm{CDCl}_{3}$ at room temperature....................................... 28

Figure $\mathrm{S} 8{ }^{1} \mathrm{H}-\mathrm{NMR}$ spectrum of $1 \mathrm{c}$ in $\mathrm{CDCl}_{3}$ at room temperature ........................................... 29 


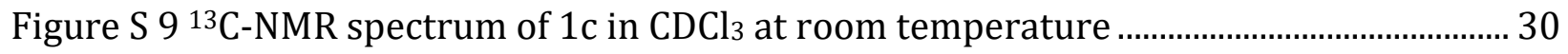

Figure S 10 HSQC-NMR spectrum of $1 \mathrm{c}$ in $\mathrm{CDCl}_{3}$ at room temperature .................................... 31

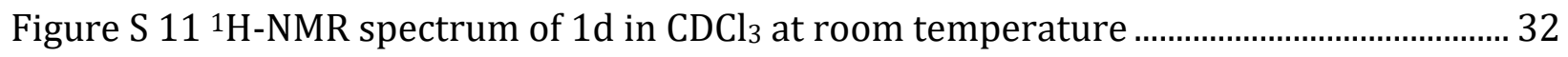

Figure $\mathrm{S} 12{ }^{13} \mathrm{C}$-NMR spectrum of $1 \mathrm{~d}$ in $\mathrm{CDCl}_{3}$ at room temperature ......................................... 33

Figure S 13 HSQC-NMR spectrum of $1 \mathrm{~d}$ in $\mathrm{CDCl}_{3}$ at room temperature ..................................... 34

Figure S 14 COSY-NMR spectrum of $1 \mathrm{~d}$ in $\mathrm{CDCl}_{3}$ at room temperature ..................................... 35

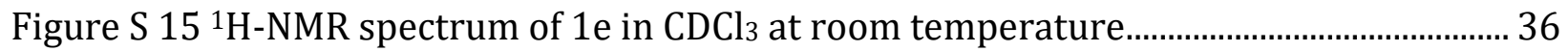

Figure $\mathrm{S} 16{ }^{13} \mathrm{C}-\mathrm{NMR}$ spectrum of $1 \mathrm{e}$ in $\mathrm{CDCl}_{3}$ at room temperature ......................................... 37

Figure S 17 HSQC-NMR spectrum of $1 \mathrm{e}$ in $\mathrm{CDCl}_{3}$ at room temperature ...................................... 38

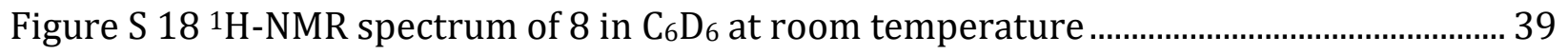

Figure $\mathrm{S} 19{ }^{13} \mathrm{C}-\mathrm{NMR}$ spectrum of 8 in $\mathrm{C}_{6} \mathrm{D}_{6}$ at room temperature .............................................. 40

Figure S 20 HSQC-NMR spectrum of 8 in $\mathrm{C}_{6} \mathrm{D}_{6}$ at room temperature......................................... 41

Figure $S 21{ }^{1} \mathrm{H}-\mathrm{NMR}$ spectrum of $2 \mathrm{a}$ in $\mathrm{C}_{6} \mathrm{D}_{6}$ at room temperature............................................ 43

Figure S $22{ }^{13} \mathrm{C}$-NMR spectrum of $2 \mathrm{a}$ in $\mathrm{C}_{6} \mathrm{D}_{6}$ at room temperature........................................... 44

Figure S 23 HSQC-NMR spectrum of $2 \mathrm{a}$ in $\mathrm{C}_{6} \mathrm{D}_{6}$ at room temperature. .................................... 45

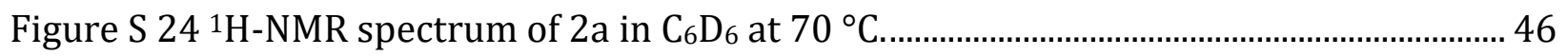

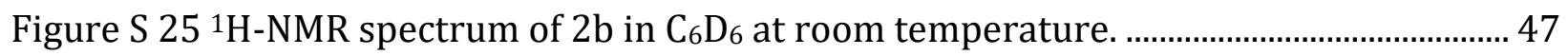

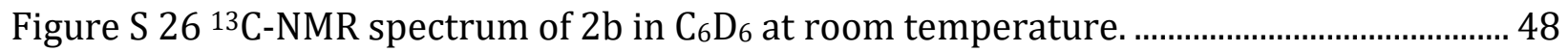

Figure S 27 HSQC-NMR spectrum of $2 \mathrm{~b}$ in $\mathrm{C}_{6} \mathrm{D}_{6}$ at room temperature....................................... 49

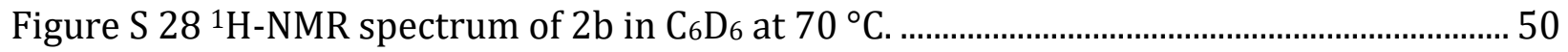

Figure S $29{ }^{1} \mathrm{H}-\mathrm{NMR}$ spectrum of $2 \mathrm{c}$ in $\mathrm{C}_{6} \mathrm{D}_{6}$ at room temperature.......................................... 51

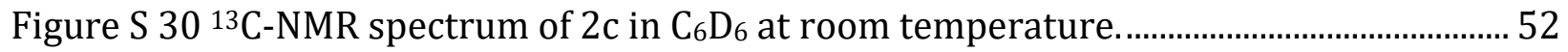

Figure S 31 HSQC-NMR spectrum of $2 \mathrm{c}$ in $\mathrm{C}_{6} \mathrm{D}_{6}$ at room temperature........................................53

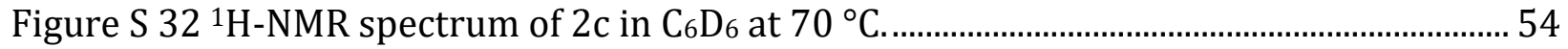

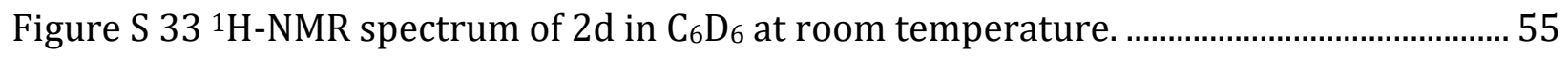

Figure S $34{ }^{13} \mathrm{C}$-NMR spectrum of $2 \mathrm{~d}$ in $\mathrm{C}_{6} \mathrm{D}_{6}$ at room temperature. .......................................... 56

Figure S 35 HSQC-NMR spectrum of $2 \mathrm{~d}$ in $\mathrm{C}_{6} \mathrm{D}_{6}$ at room temperature..................................... 57

Figure S 36 COSY-NMR spectrum of $2 \mathrm{~d}$ in $\mathrm{C}_{6} \mathrm{D}_{6}$ at room temperature........................................ 58

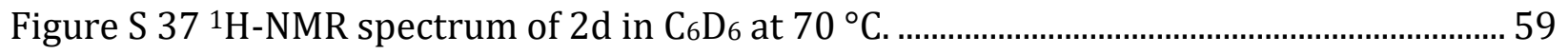

Figure $\mathrm{S} 38$ NOESY-NMR spectrum of $2 \mathrm{~d}$ in $\mathrm{C}_{6} \mathrm{D}_{6}$ at room temperature.................................. 60

Figure $S 39{ }^{1} \mathrm{H}-\mathrm{NMR}$ spectrum of $3 \mathrm{~d}$ in $\mathrm{C}_{6} \mathrm{D}_{6}$ at room temperature. ........................................... 61

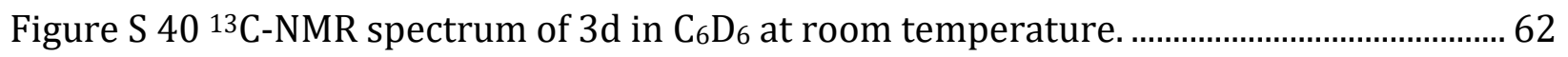

Figure S 41 HSQC-NMR spectrum of $3 \mathrm{~d}$ in $\mathrm{C}_{6} \mathrm{D}_{6}$ at room temperature..................................... 63

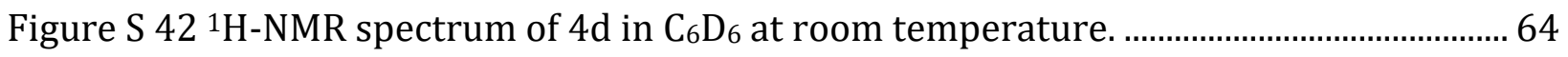




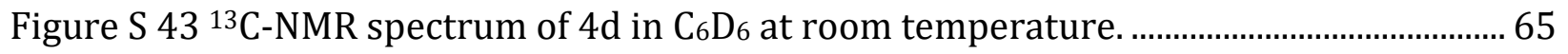

Figure S 44 HSQC-NMR spectrum of $4 \mathrm{~d}$ in $\mathrm{C}_{6} \mathrm{D}_{6}$ at room temperature..................................... 66

Figure S 45 COSY-NMR spectrum of $4 \mathrm{~d}$ in $\mathrm{C}_{6} \mathrm{D}_{6}$ at room temperature........................................ 67

Figure S 46 NOESY-NMR spectrum of $4 \mathrm{~d}$ in $\mathrm{C}_{6} \mathrm{D}_{6}$ at room temperature.................................. 68

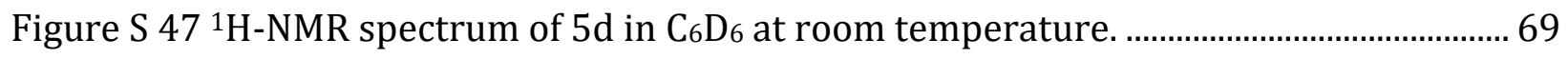

Figure $\mathrm{S} 48{ }^{13} \mathrm{C}$-NMR spectrum of $5 \mathrm{~d}$ in $\mathrm{C}_{6} \mathrm{D}_{6}$ at room temperature. ........................................ 70

Figure S 49 HSQC-NMR spectrum of $5 \mathrm{~d}$ in $\mathrm{C}_{6} \mathrm{D}_{6}$ at room temperature..................................... 71

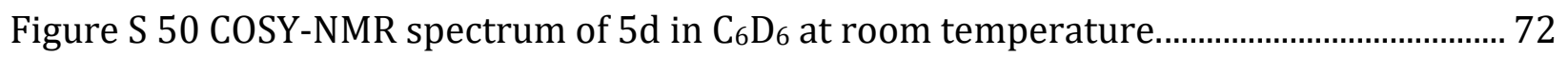

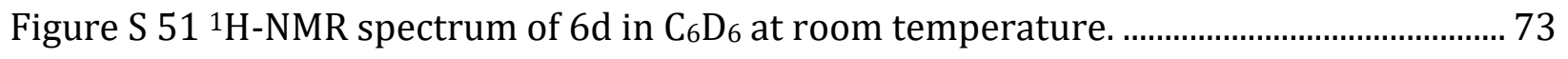

Figure $S 52{ }^{13} \mathrm{C}-\mathrm{NMR}$ spectrum of $6 \mathrm{~d}$ in $\mathrm{C}_{6} \mathrm{D}_{6}$ at room temperature. ......................................... 74

Figure $\mathrm{S} 53$ HSQC-NMR spectrum of $6 \mathrm{~d}$ in $\mathrm{C}_{6} \mathrm{D}_{6}$ at room temperature..................................... 75

Figure S 54 COSY-NMR spectrum of $6 \mathrm{~d}$ in $\mathrm{C}_{6} \mathrm{D}_{6}$ at room temperature........................................ 75 


\section{General Considerations}

Operations were performed using standard Schlenk techniques or in a VAC HE-63P nitrogen filled glovebox. Dry deoxygenated solvents were obtained from an Innovative Technology Pure Solve MD purification system. Benzene- $d_{6}$ and dichloromethane- $d_{2}$ were degassed by the freeze-pump-thaw method and dried over activated $4 \AA$ molecular sieves. NMR data was acquired in a Varian Direct Drive 400, an Agilent MR 400 or a Bruker Avance III 500 instruments and processed using MestReNova software. Mass spectrometry services were provided by the UT Austin Mass Spectrometry Facility. IR spectra were obtained in a Nicolet iS50 FT-IR using attenuated total reflectance (ATR). Microanalysis (C, H, N) were performed by Midwest Microlab Inc. Indianapolis, IN. Bis(2-aminophenyl)amine was prepared according to a previously reported procedure. ${ }^{1}$

\section{Crystallographic details}

Single crystals were coated in paratone oil and mounted on nylon loops. Data was collected in the following diffractometers:

- A Rigaku AFC12 diffractometer with a Saturn 724+ CCD using graphite

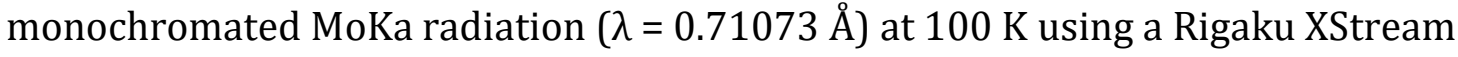
low temperature device. Data Reduction was performed with Rigaku's Crystal Clear version 14.0.

- $\quad$ An Agilent Supernova diffractometer equipped with an AtlasS2 CCD using a collimating mirror monochromator with $\mu$-focus CuKa radiation $(\lambda=1.5418 \AA)$ at $100 \mathrm{~K}$ provided by an Oxford 700 series Cryostream device. Data was reduced with Agilent's CrysAlysPro software.

Structures were solved using Olex $2^{2}$ by SHELXS ${ }^{3}$ or SHELXT ${ }^{4}$ and refined with full-matrix least-squares calculations of $F^{2}$ by usong SHELXL ${ }^{5}$. Additional refinement details can be found in the corresponding CIF files. ORTEP diagrams were created using Olex2. 
Figure $\mathbf{S} 1$ Solid-state packing of ligand 1a with hydrogen bonding interactions (dash lines). Non hydrogen bonding hydrogens removed for clarity.

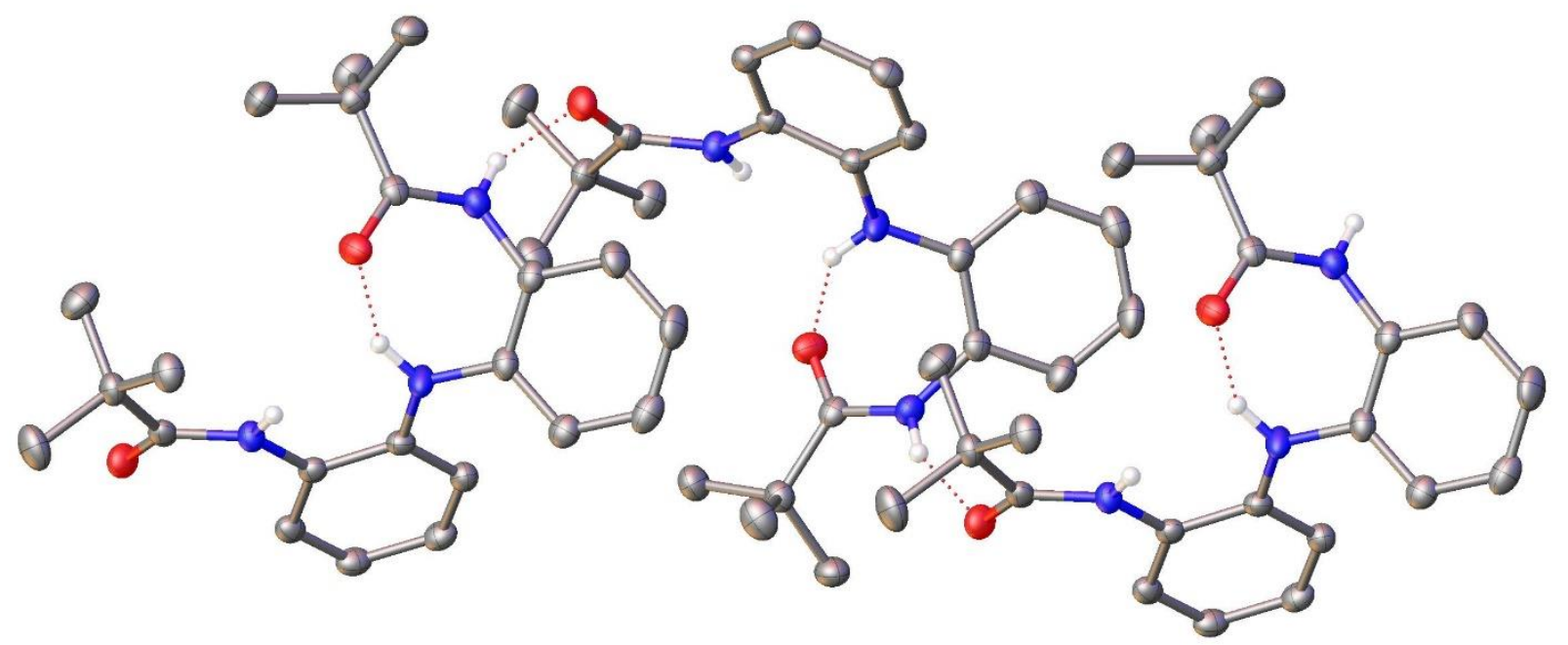




\section{Ligand Synthesis \\ Bis(2-pivaloylamidophenyl)amine $\left[\mathrm{C}_{22} \mathrm{H}_{29} \mathrm{~N}_{3} \mathrm{O}_{2}\right]$ (1a)}

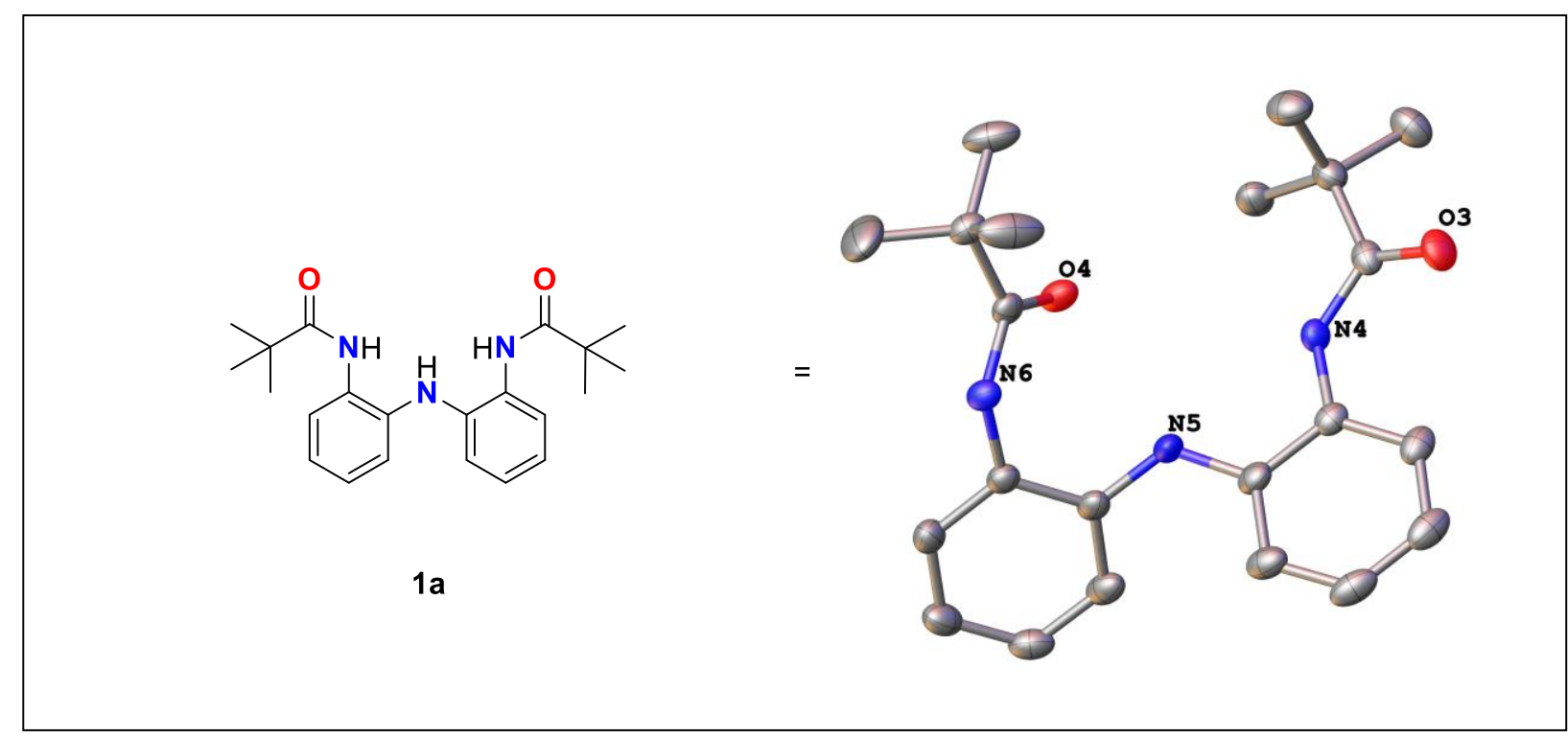

A solution of bis(2-aminophenyl)amine $(2.0 \mathrm{~g}, 10 \mathrm{mmol})$ in dichloromethane $(100 \mathrm{~mL})$ was supplemented with triethylamine $(3.1 \mathrm{~mL}, 22.1 \mathrm{mmol})$ and cooled to $0{ }^{\circ} \mathrm{C}$. Pivaloyl chloride was added via syringe $(2.7 \mathrm{~mL}, 22.1 \mathrm{mmol})$. The reaction mixture was stirred overnight while warming to room temperature. The organic phase was washed with saturated $\mathrm{NaHCO}_{3}(3 \mathrm{x}$ $100 \mathrm{~mL}$ ) and dried over $\mathrm{Na}_{2} \mathrm{SO}_{4}$. Recrystallization from dichloromethane/hexanes (1:1) yields an off-white powder (2.9 g, $79 \%$ ). XRD quality crystals were grown by slow evaporation of a dichloromethane solution.

${ }^{1}$ H-NMR (500 MHz, Chloroform-d) $\delta 7.71-7.65$ (m, 4H, Aryl-CH + Amide-NH), 7.04 (dtd, $J$ $=23.7,7.5,1.6 \mathrm{~Hz}, 4 \mathrm{H}$, Aryl $-H), 6.88(\mathrm{dd}, J=7.8,1.6 \mathrm{~Hz}, 2 \mathrm{H}, \operatorname{Aryl}-H), 6.13(\mathrm{~s}, 1 \mathrm{H},-\mathrm{N} H), 1.20(\mathrm{~s}$, $\left.18 \mathrm{H},-\mathrm{C}\left(\mathrm{CH}_{3}\right)_{3}\right)$.

${ }^{13}$ C-NMR (126 MHz, Chloroform-d) $\delta 177.3(C=0), 135.8\left(\mathrm{C}_{\mathrm{q}}\right), 129.3\left(\mathrm{C}_{\mathrm{q}}\right), 126.0$ (Aryl- $C \mathrm{H}$ ), 123.5 (Aryl- $\mathrm{CH}), 123.0$ (Aryl- $\mathrm{CH}), 121.0$ (Aryl- $\mathrm{CH}), 39.5\left(-\mathrm{C}\left(\mathrm{CH}_{3}\right)_{3}\right), 27.5\left(-\mathrm{C}\left(\mathrm{CH}_{3}\right)_{3}\right)$.

HRMS-ESI m/z 390.2158 (Calculated $\mathrm{C}_{22} \mathrm{H}_{29} \mathrm{~N}_{3} \mathrm{NaO}_{2}{ }^{+} \mathrm{m} / \mathrm{z} 390.2152$ ).

FT-IR (ATR, $\mathbf{~ c m}^{-1}$ ): 3413, 3294, 2962, 1654, 1599, 1502, 1482, 1453, 1429, 1396, 1365, $1296,1263,1225,1167,1104,1042,926,894,791,764,749,649,586,565,440$.

Melting Point: $147^{\circ} \mathrm{C}$. 


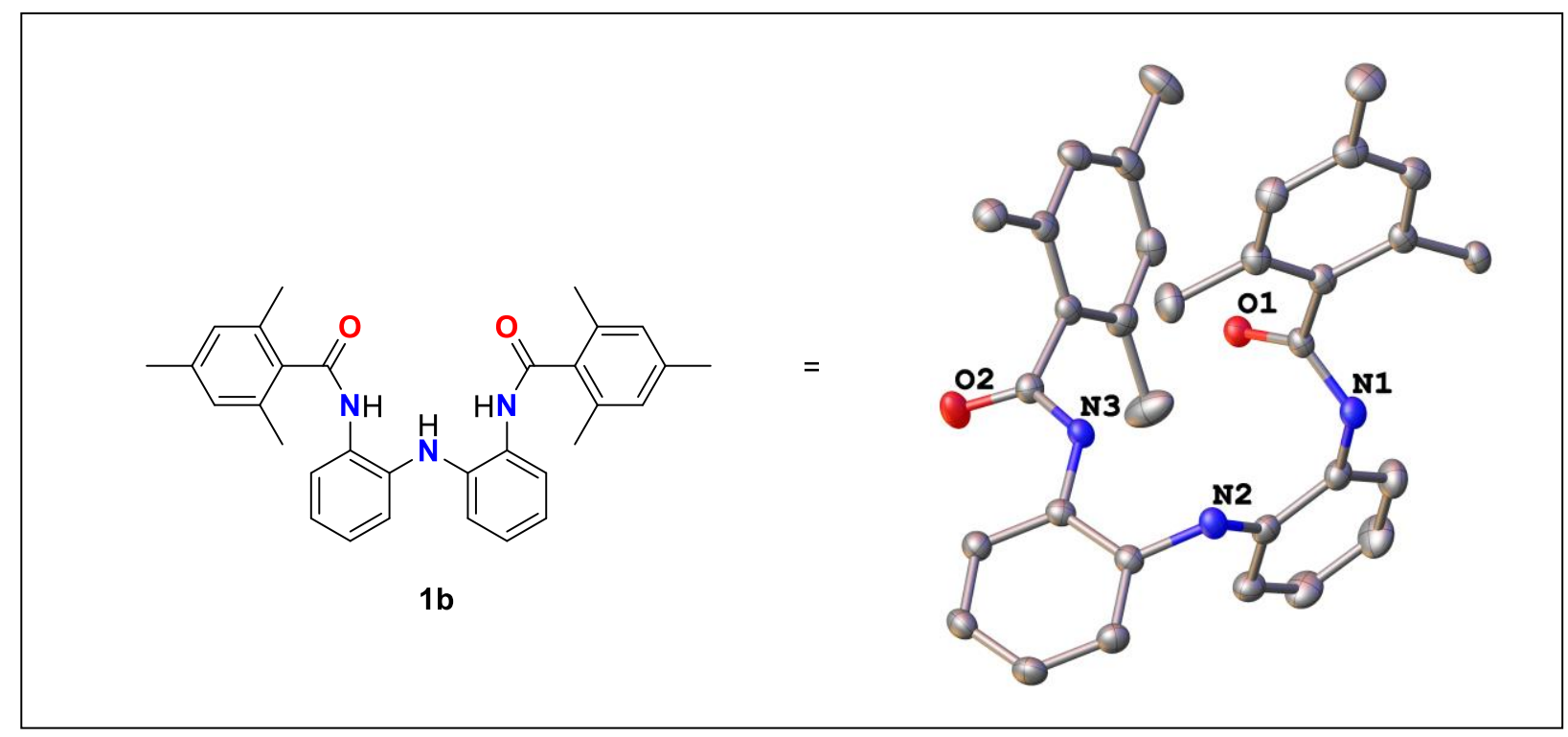

To a solution of bis(2-aminophenyl)amine $(2.0 \mathrm{~g}, 10 \mathrm{mmol})$ and triethylamine $(3.1 \mathrm{~mL}, 22.1$ $\mathrm{mmol}$ ) in dichloromethane $(100 \mathrm{~mL})$ mesitoyl chloride $(3.7 \mathrm{~mL}, 22.1 \mathrm{mmol})$ was added via syringe at $0{ }^{\circ} \mathrm{C}$. The reaction was allowed to attain room temperature overnight. The organic phase was washed with saturated $\mathrm{NaHCO}_{3}(3 \times 100 \mathrm{~mL})$ and dried over $\mathrm{Na}_{2} \mathrm{SO}_{4}$. Solvent was removed and the resulting oil was dissolved in ether. Upon standing a tan solid is obtained. The solid was filtered and washed with copious amounts of ether to afford the ligand as a white solid. Yield (2.0 g, $40 \%)$. XRD quality crystals were grown by reverse vapor diffusion of acetonitrile into water.

${ }^{1}$ H-NMR (500 MHz, Chloroform-d) $\delta 7.86$ (s, 2H, Amide-NH), 7.80 (dd, $J=7.9,1.7 \mathrm{~Hz}, 2 \mathrm{H}$, Aryl $-H$ ), 7.10 (dtd, $J=21.2,7.5,1.6 \mathrm{~Hz}, 4 \mathrm{H}$, Aryl $-H$ ), 6.93 (dd, $J=7.8,1.7 \mathrm{~Hz}, 2 \mathrm{H}$, Aryl $-H$ ), 6.77 $\left(\mathrm{s}, 4 \mathrm{H}\right.$, Aryl- $H$ ) $, 6.20(\mathrm{~s}, 1 \mathrm{H},-\mathrm{N} H), 2.24\left(\mathrm{~s}, 6 \mathrm{H}\right.$, Aryl- $\left.\mathrm{CH}_{3}\right), 2.14\left(\mathrm{~s}, 12 \mathrm{H}, \operatorname{Aryl}-\mathrm{CH}_{3}\right)$.

${ }^{13}$ C-NMR (126 MHz, Chloroform-d) $\delta 169.3(C=0), 138.7\left(C_{q}\right), 136.3\left(C_{q}\right), 134.4\left(C_{q}\right), 134.2$ $\left(\mathrm{C}_{\mathrm{q}}\right), 129.7\left(\mathrm{C}_{\mathrm{q}}\right), 128.2(\operatorname{Aryl} \mathrm{CH}), 126.4(\operatorname{Aryl} \mathrm{CH}), 123.5$ (Aryl-CH), 123.1 (Aryl-CH), 122.0 (Aryl- $\mathrm{CH}), 21.1\left(\right.$ Aryl- $\left.\mathrm{CH}_{3}\right), 19.0\left(\operatorname{Aryl}-\mathrm{CH}_{3}\right)$.

HRMS-ESI m/z 492.2651 (Calculated $\mathrm{C}_{32} \mathrm{H}_{34} \mathrm{~N}_{3} \mathrm{O}_{2}{ }^{+} \mathrm{m} / \mathrm{z}$ 492.2646).

FT-IR (ATR, $\mathbf{c m}^{-1}$ ): 3382, 3174, 1640, 1599, 1525, 1503, 1476, 1449, 1413, 1311, 1293, 1171, 1106, 1092, 1043, 909, 882, 854, 761, 741, 702, 616, 601, 542, 530, 499, 456.

Melting Point: $220-221^{\circ} \mathrm{C}$. 


\section{Bis(2-adamantoylamidophenyl)amine $\left[\mathrm{C}_{34} \mathrm{H}_{41} \mathrm{~N}_{3} \mathrm{O}_{2}\right]$ (1c)}

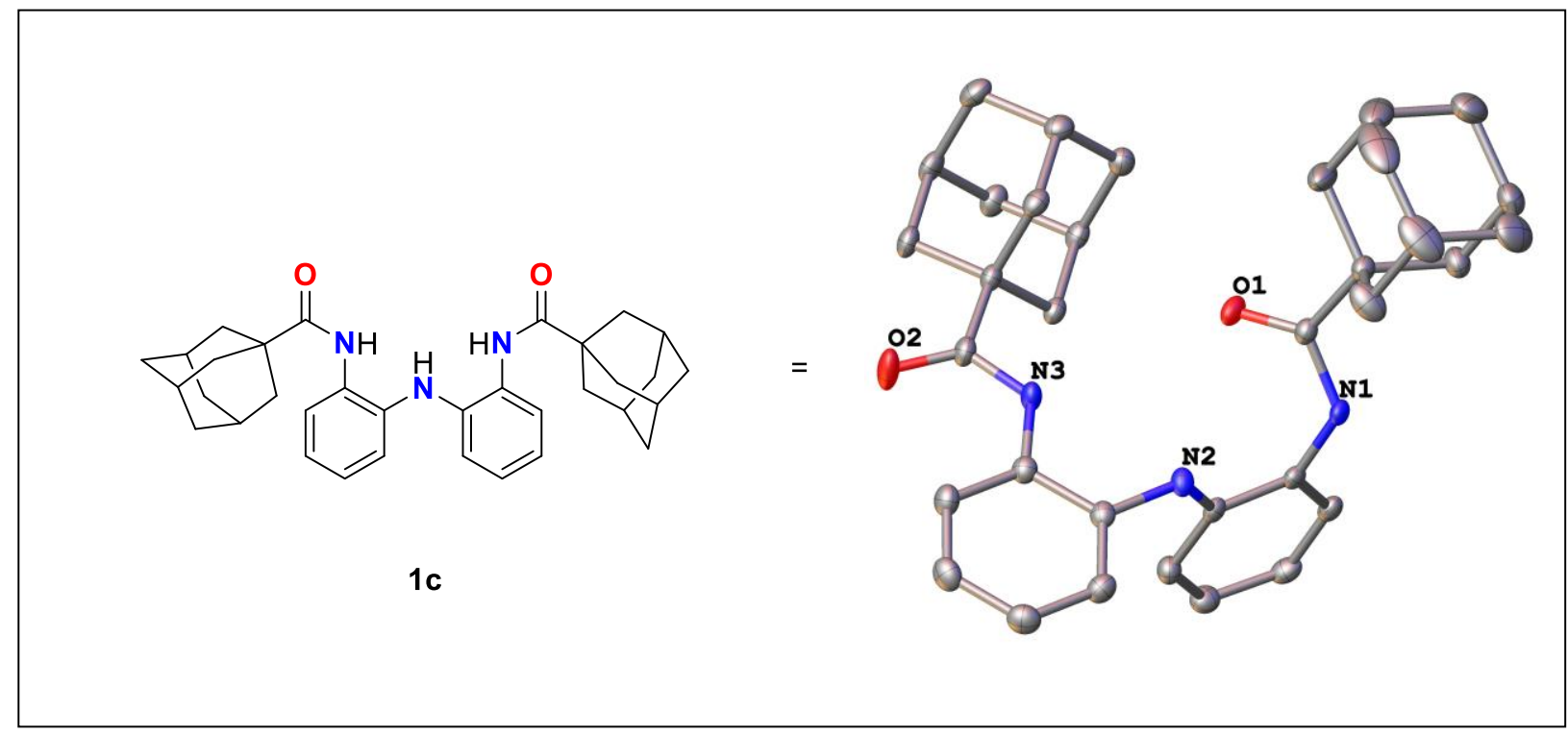

Adamantoyl chloride (4.38 g, $22.1 \mathrm{mmol})$ in dichloromethane (40 $\mathrm{mL})$ was cannula transferred to a dichloromethane solution $(80 \mathrm{~mL})$ containing bis(2-aminophenyl)amine $(2.0 \mathrm{~g}, 10 \mathrm{mmol})$ and triethylamine $(22.1 \mathrm{mmol}, 3.1 \mathrm{ml})$ at $0{ }^{\circ} \mathrm{C}$. The mixture was allowed to warm to room temperature and stirred overnight. The organic phase was washed with 0.5 $\mathrm{M} \mathrm{HCl}\left(3 \times 100 \mathrm{~mL}\right.$ ), saturated $\mathrm{NaHCO}_{3}(3 \times 100 \mathrm{~mL})$ and dried over $\mathrm{Na}_{2} \mathrm{SO}_{4}$. Solvent was evaporated under vacuum and the resulting pink solid was crushed and washed with several portions of ether ( $5 \times 5 \mathrm{~mL}$ ) to afford a white crystalline solid (3.79 g, $72 \%$ ). Crystals suitable for XRD were grown by vapor diffusion of pentane into a THF solution.

${ }^{1}$ H-NMR (500 MHz, Chloroform-d) $\delta 7.71(\mathrm{dd}, J=7.8,1.6 \mathrm{~Hz}, 2 \mathrm{H}$, Aryl- $H$ ), $7.63(\mathrm{~s}, 2 \mathrm{H}$, Amide$\mathrm{N} H$ ), 7.04 (dtd, $J=22.1,7.5,1.6 \mathrm{~Hz}, 4 \mathrm{H}$, Aryl- $H$ ), 6.92 (dd, $J=7.8,1.6 \mathrm{~Hz}, 2 \mathrm{H}$, Aryl- $H$ ), 6.09 (s, $1 \mathrm{H}, \mathrm{N}-H$ ) 2.03 (p, J = 3.3 Hz, 6H, $-\mathrm{CH}$ ), $1.86\left(\mathrm{~d}, J=2.8 \mathrm{~Hz}, 12 \mathrm{H},-\mathrm{CH} \mathrm{H}^{-}\right), 1.77-1.63(\mathrm{~m}, 12 \mathrm{H},-$ $\mathrm{CH}_{2}-\mathrm{O}$.

${ }^{13}$ C-NMR (126 MHz, Chloroform-d) $\delta 176.7(C=0), 135.6\left(\mathrm{C}_{\mathrm{q}}\right), 129.2\left(\mathrm{C}_{\mathrm{q}}\right), 125.8$ (Aryl-CH), 123.4 (Aryl-CH), 122.9 (Aryl- $\mathrm{CH}$ ), 121.0 (Aryl- $\mathrm{CH}$ ), 41.5 (Cq), $39.1\left(-\mathrm{CH}_{2}-\right), 36.4\left(-\mathrm{CH}_{2}-\right), 28.1$ $(-\mathrm{CH})$.

HRMS-ESI m/z 546.3097 (Calculated $\mathrm{C}_{34} \mathrm{H}_{41} \mathrm{~N}_{3} \mathrm{NaO}_{2}{ }^{+} \mathrm{m} / \mathrm{z}$ 546.3091).

FT-IR (ATR, cm-1): 3302, 2898, 2848, 1645, 1593, 1504, 1445, 1371, 1344, 1297, 1259, $1230,1179,1158,1104,1076,1040,976,941,919,895,850,799,751,675,639,594,583$, $567,484,453,444,426$.

Melting Point: $237-238{ }^{\circ} \mathrm{C}$ 


\section{Bis(2,4,6-(tris(Isopropyl)benzoylphenyl))amine $\left[\mathrm{C}_{44} \mathrm{H}_{57} \mathrm{~N}_{3} \mathrm{O}_{2}\right]$ (1d)}

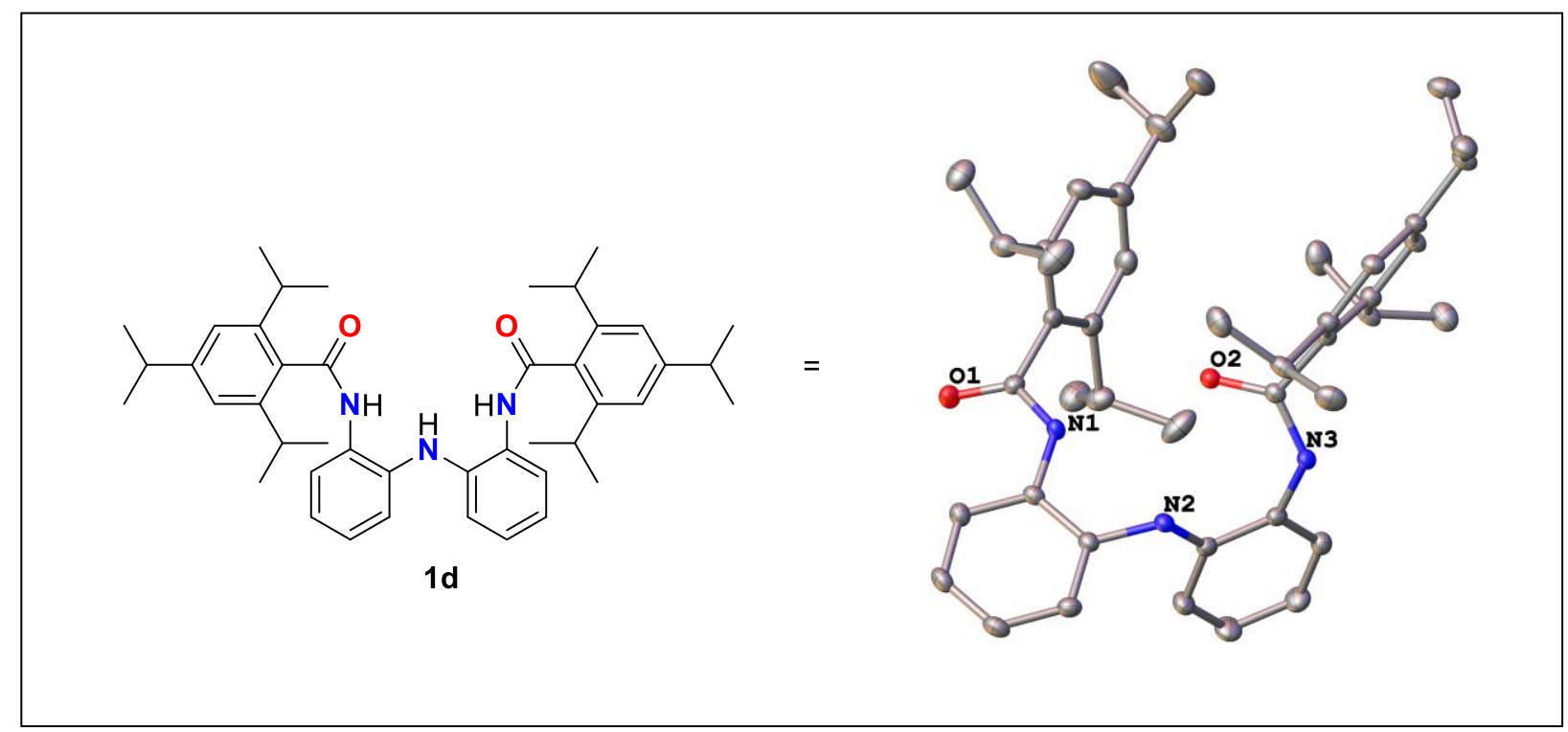

2,4,6-tris(isopropyl)benzoyl chloride (4.45 g, $16.7 \mathrm{mmol}$ ) in dichloromethane (20 $\mathrm{mL}$ ) was cannula transferred to a flask containing a dichloromethane solution $(20 \mathrm{~mL})$ of bis(2aminophenyl)amine $(1.57 \mathrm{~g}, 7.9 \mathrm{mmol})$ and triethylamine $(2.4 \mathrm{~mL}, 17 \mathrm{mmol})$ at $0{ }^{\circ} \mathrm{C}$. The mixture was allowed to warm to room temperature and stirred for $48 \mathrm{~h}$. Additional dichloromethane $(80 \mathrm{~mL})$ was added to fully dissolve the contents of the flask. The organic phase was washed with water $(3 \times 100 \mathrm{~mL})$ followed by saturated sodium bicarbonate $(3 \mathrm{x}$ $100 \mathrm{~mL}$ ) solution and lastly with brine $(100 \mathrm{~mL})$. Dried over $\mathrm{Na}_{2} \mathrm{SO}_{4}$ and the solvent was evaporated under reduced pressure to afford a tan solid. The solid was recrystallized twice from diethyl ether/hexanes $(1: 3,15 \mathrm{~mL}$ ) (Silica, $\mathrm{Rf}=0.31$.) Yield of white powder $(2.44 \mathrm{~g}, 47$ $\%)$. XRD crystals were grown by reverse vapor diffusion of acetonitrile solution into water.

1H-NMR (500 MHz, Chloroform-d) $\delta 7.85$ (dd, $J=7.3,2.1 \mathrm{~Hz}, 2 \mathrm{H}$, Aryl- $H$ ), 7.77 (s, 2H, Amide$\mathrm{N} H$ ), 7.13 (m, 4H, Aryl-H), 6.97 (s, 4H, Aryl-H), 6.91 (dd, J = 7.2, 2.2 Hz, 2H, Aryl-H), 6.46 (s, $1 \mathrm{H}, \mathrm{N}-\mathrm{H}$ ), 2.97 (hept, $J=6.9 \mathrm{~Hz}, 4 \mathrm{H}, \mathrm{Ar}-\mathrm{CH}\left(\mathrm{CH}_{3}\right)_{2}$ ), 2.86 (hept, $J=7.0 \mathrm{~Hz}, 2 \mathrm{H}, \mathrm{Ar}-\mathrm{CH}\left(\mathrm{CH}_{3}\right)_{2}$ ), $1.22\left(\mathrm{~d}, J=6.9 \mathrm{~Hz}, 12 \mathrm{H}, \mathrm{Ar}-\mathrm{CH}\left(\mathrm{CH}_{3}\right)_{2}\right), 1.18\left(\mathrm{br}, 6 \mathrm{H}, \mathrm{Ar}-\mathrm{CH}\left(\mathrm{CH}_{3}\right)_{2}\right), 1.06\left(\mathrm{br}, 6 \mathrm{H}, \mathrm{Ar}-\mathrm{CH}\left(\mathrm{CH}_{3}\right)_{2}\right)$.

${ }^{13}$ C-NMR (126 MHz, Chloroform-d) $\delta 169.7(C=0), 150.1\left(C_{q}\right), 144.9\left(C_{q}\right), 136.5\left(C_{q}\right), 133.11$ $\left(\mathrm{C}_{\mathrm{q}}\right), 130.2\left(\mathrm{C}_{\mathrm{q}}\right), 126.5$ (Aryl- $\left.\mathrm{CH}\right), 123.8$ (Aryl-CH), 122.7 (Aryl-CH), 122.1 (Aryl-CH), 121.0 (Aryl- $\mathrm{CH}), 34.4\left(\mathrm{Ar}-\mathrm{CH}\left(\mathrm{CH}_{3}\right)_{2}\right), 31.2\left(\mathrm{Ar}-\mathrm{CH}\left(\mathrm{CH}_{3}\right)_{2}\right), 24.7\left(\mathrm{Ar}-\mathrm{CH}\left(\mathrm{CH}_{3}\right) 2\right), 23.9\left(\mathrm{Ar}-\mathrm{CH}\left(\mathrm{CH}_{3}\right)_{2}\right)$.

HRMS-ESI m/z 682.4340 (Calculated $\mathrm{C}_{44} \mathrm{H}_{57} \mathrm{~N}_{3} \mathrm{NaO}_{2}{ }^{+} \mathrm{m} / \mathrm{z}$ 682.4343).

FT-IR (ATR, cm-1): 3203, 2957, 2868, 1641, 1605, 1533, 1512, 1456, 1435, 1385, 1363, $1346,1310,1171,1156,1107,1069,1042,938,907,876,801,754,740,655,584,512,456$.

Melting Point: $216-217^{\circ} \mathrm{C}$. 


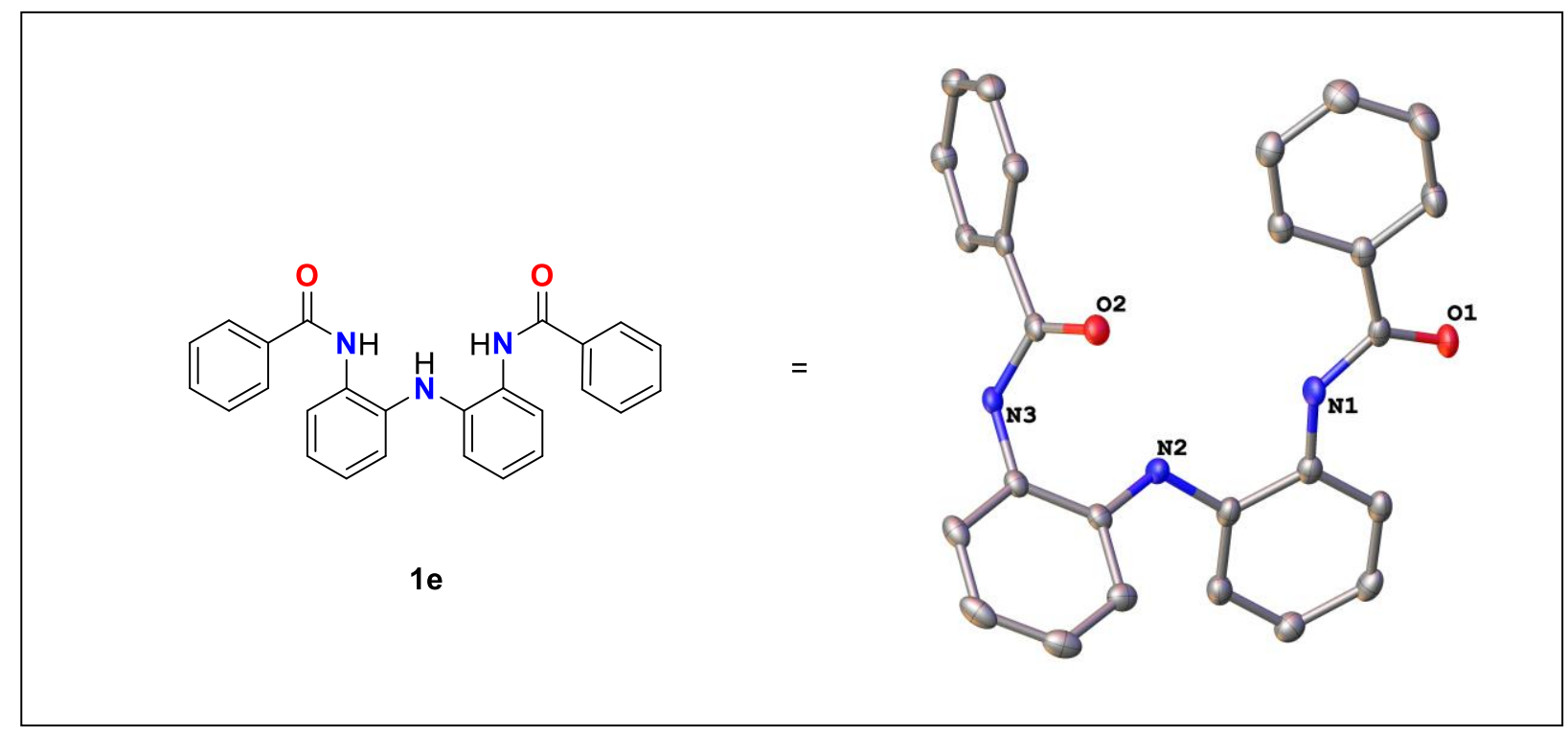

To a solution of bis(2-aminophenyl)amine $(2.0 \mathrm{~g}, 10 \mathrm{mmol})$ and triethylamine $(3.1 \mathrm{~mL}, 22.1$ $\mathrm{mmol}$ ) in dichloromethane $(100 \mathrm{~mL})$; benzoyl chloride $(2.6 \mathrm{~mL}, 22.1 \mathrm{mmol})$ was added via syringe at $0{ }^{\circ} \mathrm{C}$. The reaction mixture was allowed to attain room temperature and it was stirred overnight. The organic phase was washed with saturated $\mathrm{NaHCO}_{3}$ solution $(3 \times 100$ $\mathrm{mL}$ ) and dried over $\mathrm{Na}_{2} \mathrm{SO}_{4}$. Recrystallization from dichloromethane/hexanes (2:8) yields an off white microcrystalline solid (3.24 g, 80\%). XRD quality crystals were grown by layering hexanes into a concentrated solution of dichloromethane and cooling to $-25^{\circ} \mathrm{C}$.

1H-NMR (500 MHz, DMSO-d6) $\delta 9.96$ (s, 2H, N-H), 7.88 - 7.82 (m, 4H, Aryl- $H$ ), 7.61 - 7.53 $(\mathrm{m}, 2 \mathrm{H}$, Aryl $-H), 7.56-7.42(\mathrm{~m}, 6 \mathrm{H}$, Aryl $-H), 7.24$ (s, $1 \mathrm{H}, \mathrm{N}-H), 7.20-7.08$ (m, 4H, Aryl $-H$ ), $6.95(\mathrm{td}, J=7.4,1.8 \mathrm{~Hz}, 2 \mathrm{H}$, Aryl $-H)$.

${ }^{13}$ C-NMR (126 MHz, DMSO-d6) $\delta 166.0(C=0), 138.4\left(C_{q}\right), 134.6\left(C_{q}\right), 131.9$ (Aryl- $H$ ), 128.7 $\left(\mathrm{C}_{\mathrm{q}}\right), 128.6$ (Aryl $-H$ ), 128.3 (Aryl- $H$ ), 127.1 (Aryl- $H$ ), 126.8 (Aryl- $H$ ), 121.2 (Aryl- $H$ ), 119.1 (Aryl-H).

HRMS-ESI m/z 408.1716 (Calculated $\mathrm{C}_{26} \mathrm{H}_{22} \mathrm{~N}_{3} \mathrm{O}_{2}{ }^{+} \mathrm{m} / \mathrm{z}$ 408.1707).

FT-IR (ATR, cm$^{-1}$ ): 3360, 3267, 3062, 1669, 1643, 1602, 1591, 1579, 1517, 1495, 1449, 1437, 1301, 1252, 1177, 1112, 1072, 1043, 1025, 1001, 929, 913, 882, 794, 747, 729, 715, $701,683,647,595,574,542,499,460,445$.

Melting Point: $173-174^{\circ} \mathrm{C}$. 


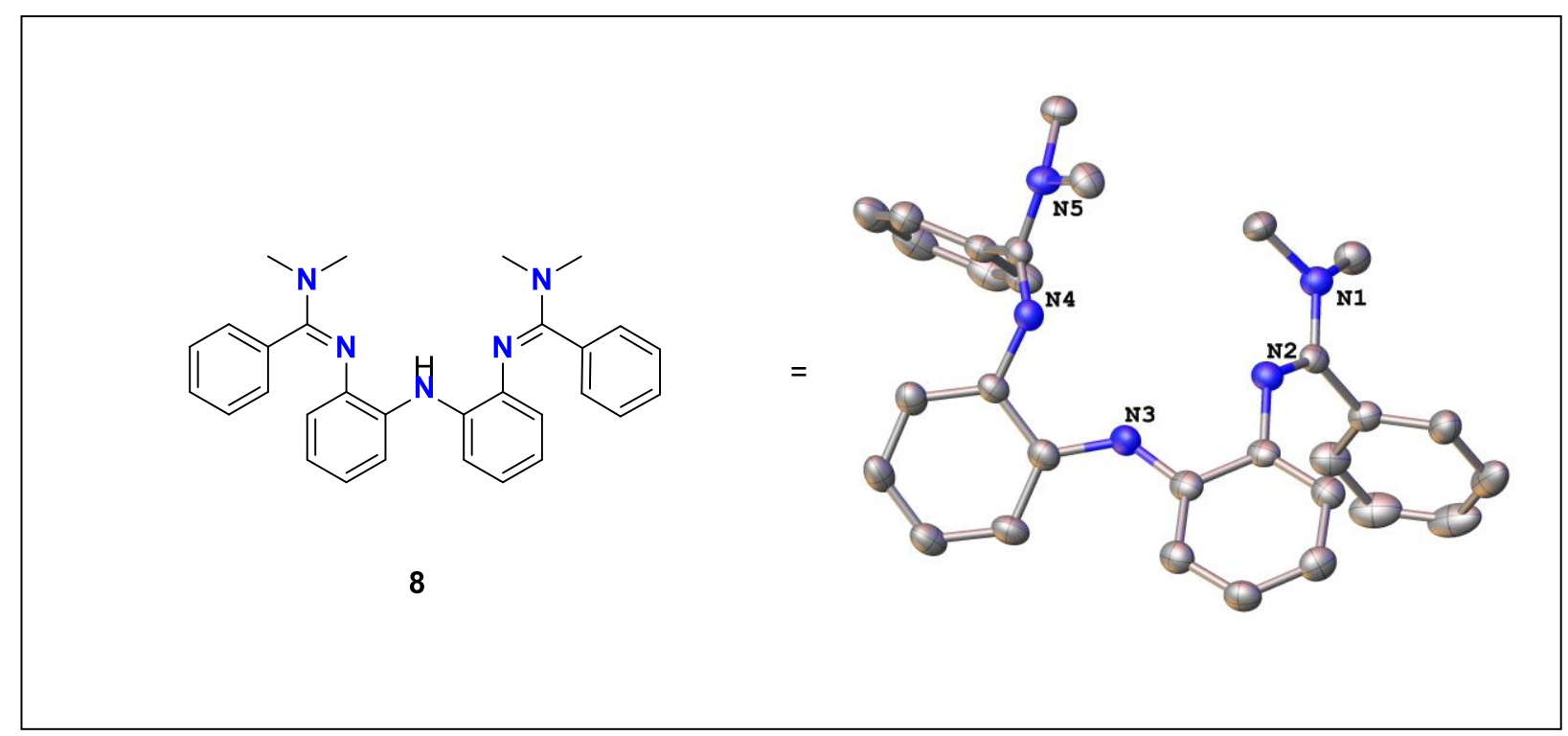

To a suspension of $1 \mathbf{e}(1.5 \mathrm{~g}, 3.7 \mathrm{mmol})$ in tetrahydrofuran $\left.(30 \mathrm{~mL}) \mathrm{Ti}\left(\mathrm{NMe}_{2}\right) 4\right)(4.4 \mathrm{~mL}, 18.4$ $\mathrm{mmol}$ ) was added. The mixture was refluxed for $84 \mathrm{~h}$. After cooling to room temperature, the orange suspension was carefully quenched by dropwise addition of wet tetrahydrofuran $(100 \mathrm{~mL})$ and stirred for 1 hour. The suspension was filtered on a fritted funnel and the $\mathrm{TiO}_{2}$ cake washed with dichloromethane $(50 \mathrm{~mL})$. Solvent was removed under reduced pressure and upon purification via chromatography title compound was obtained as a yellow crystalline solid. Yield (602 mg, $36 \%$ ). Single crystals were grown by slow evaporation of a toluene/hexane solution.

${ }_{1}^{1}$ H-NMR (500 MHz, Benzene- $\left.d 6\right) \delta 7.54(\mathrm{dd}, J=8.0,1.3 \mathrm{~Hz}, 2 \mathrm{H}$, Aryl- $H$ ), $7.38(\mathrm{~s}, 1 \mathrm{H}, \mathrm{N}-H$ ), 7.11- 7.05 (m, 4H, Aryl- $H$ ), 6.89 (dd, $J=8.1,6.4 \mathrm{~Hz}, 4 \mathrm{H}$, Aryl $-H$ ), $6.88-6.77$ (m, 4H, Aryl $H$ ), $6.66-6.54(\mathrm{~m}, 4 \mathrm{H}$, Aryl $-H), 2.76\left(\mathrm{br}, 12 \mathrm{H},-\mathrm{N}\left(\mathrm{CH}_{3}\right)_{2}\right)$.

${ }^{13}$ C-NMR (126 MHz, Benzene-d6) $\delta 161.3$ (amidine-C), 140.8 (Cq), 136.7 (Cq), 134.1 (Cq), 128.3 (Aryl- $\mathrm{CH}$ ), 128.3 (Aryl- $\mathrm{CH}$ ), 128.0 (Aryl- $\mathrm{CH}$ ), 122.5 (Aryl- $\mathrm{CH}$ ), 122.0 (Aryl- $\mathrm{CH}$ ), 119.1 (Aryl- $\mathrm{CH}), 114.2($ Aryl- $\mathrm{CH}), 37.8\left(\mathrm{br},-\mathrm{N}\left(\mathrm{CH}_{3}\right)_{2}\right)$.

FT-IR (ATR, cm-1): 3359, 3330, 2922, 1605, 1857, 1569, 1506, 1475, 1444, 1456, 1386, 1339, 1296, 1279, 1268, 1219, 1138, 1103, 1080, 1026, 936, 915, 842, 821, 775, 750, 734, $694,628,588,555,528,483,464,430$.

HRMS-ESI $\mathrm{m} / \mathrm{z} 462.2658$ (Calculated $\mathrm{C}_{30} \mathrm{H}_{32} \mathrm{~N}_{5}{ }^{+} \mathrm{m} / \mathrm{z}$ 462.2652).

Melting point: $172-173^{\circ} \mathrm{C}$. 


\section{Metal Complex Synthesis}

\section{Compound 2a [ $\left.\mathrm{C}_{26} \mathrm{H}_{39} \mathrm{~N}_{5} \mathrm{O}_{2} \mathrm{Ti}\right]$}

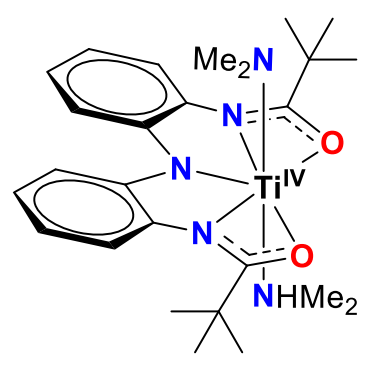

$2 a$

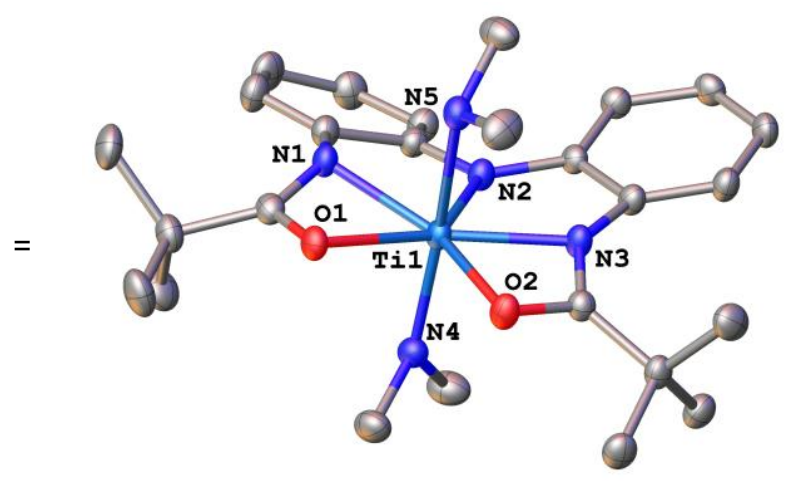

In a glovebox, to a vial containing $1 \mathrm{a}(500 \mathrm{mg}, 1.37 \mathrm{mmol})$ a solution of $\mathrm{Ti}\left(\mathrm{NMe}_{2}\right)_{4}(324 \mu \mathrm{L}$, $1.37 \mathrm{mmol})$ in diethyl ether $(7 \mathrm{~mL})$ was added via pipette. The reaction proceeds immediately turning the reaction mixture dark red. Crystalline material deposits after standing overnight. The supernatant was removed, and the crystals were washed with diethyl ether $(2 \mathrm{~mL})$. The crystalline residue was dried in vacuum to afford 2a. Yield (282 mg, $41 \%$ ). XRD quality crystals where grown by slow cooling of a concentrated ethereal solution to $-25^{\circ} \mathrm{C}$.

${ }_{1}^{1}$ H-NMR (400 MHz, Benzene- $d_{6}$ ) $\delta 7.97(\mathrm{dd}, J=8.3,1.3 \mathrm{~Hz}, 2 \mathrm{H}$, Aryl- $H$ ), 7.39 (dd, $J=7.9,1.4$ Hz, 2H, Aryl- $H$ ), $7.17-7.09$ (m, 2H, Aryl- $H$ ), 6.94 (ddd, $J=7.9,7.3,1.3 \mathrm{~Hz}, 2 \mathrm{H}$, Aryl $-H$ ), 3.08 $\left(\mathrm{s}, 6 \mathrm{H}, \mathrm{Ti}-\mathrm{N}\left(\mathrm{CH}_{3}\right)_{2}\right), 1.52\left(\mathrm{~d}, J=6.3 \mathrm{~Hz}, 6 \mathrm{H}, \mathrm{Ti}-\mathrm{NH}\left(\mathrm{CH}_{3}\right)_{2}\right), 1.37\left(\mathrm{~s}, 18 \mathrm{H},-\mathrm{C}\left(\mathrm{CH}_{3}\right)_{3}\right), 0.72(\mathrm{~m}, 1 \mathrm{H}$, -, $\left.\mathrm{Ti}-\mathrm{N} H\left(\mathrm{CH}_{3}\right)_{2}\right)$.

${ }^{13}$ C-NMR $\left(100 \mathrm{MHz}\right.$, Benzene- $\left.d_{6}\right) \delta 183.1(\mathrm{C}=0), 148.4\left(\mathrm{C}_{\mathrm{q}}\right), 134.9\left(\mathrm{C}_{\mathrm{q}}\right), 123.3$ (Aryl- $\left.\mathrm{CH}\right), 119.5$ (Aryl- $C \mathrm{H}), 119.5$ (Aryl- $\mathrm{CH}), 113.4$ (Aryl- $\mathrm{CH}), 50.4\left(\mathrm{Ti}-\mathrm{N}\left(\mathrm{CH}_{3}\right)_{2}\right), 39.8\left(\mathrm{Ti}-\mathrm{NH}\left(\mathrm{CH}_{3}\right)_{2}\right), 39.1\left(\mathrm{C}_{\mathrm{q}}\right)$, $26.0\left(-\mathrm{C}\left(\mathrm{CH}_{3}\right)_{3}\right)$.

FT-IR (ATR, cm$^{-1}$ ): 3306, 3054, 2966, 2926, 2850, 2759, 1660, 1574, 1511, 1478, 1447, $1418,1362,1298,1278,1263,1208,1167,1152,1125,1113,1054,1035,963,944,898$, $856,846,830,742,728,716,692,684,598,588,572,514,488,456,421$.

Melting Point: $110^{\circ} \mathrm{C}$ (decomposition).

Elemental Analysis Anal. Found: C, 61.90; H, 7.42; N, 13.62. Calc: C, 62.3; H, 7.84; N, 13.97. 


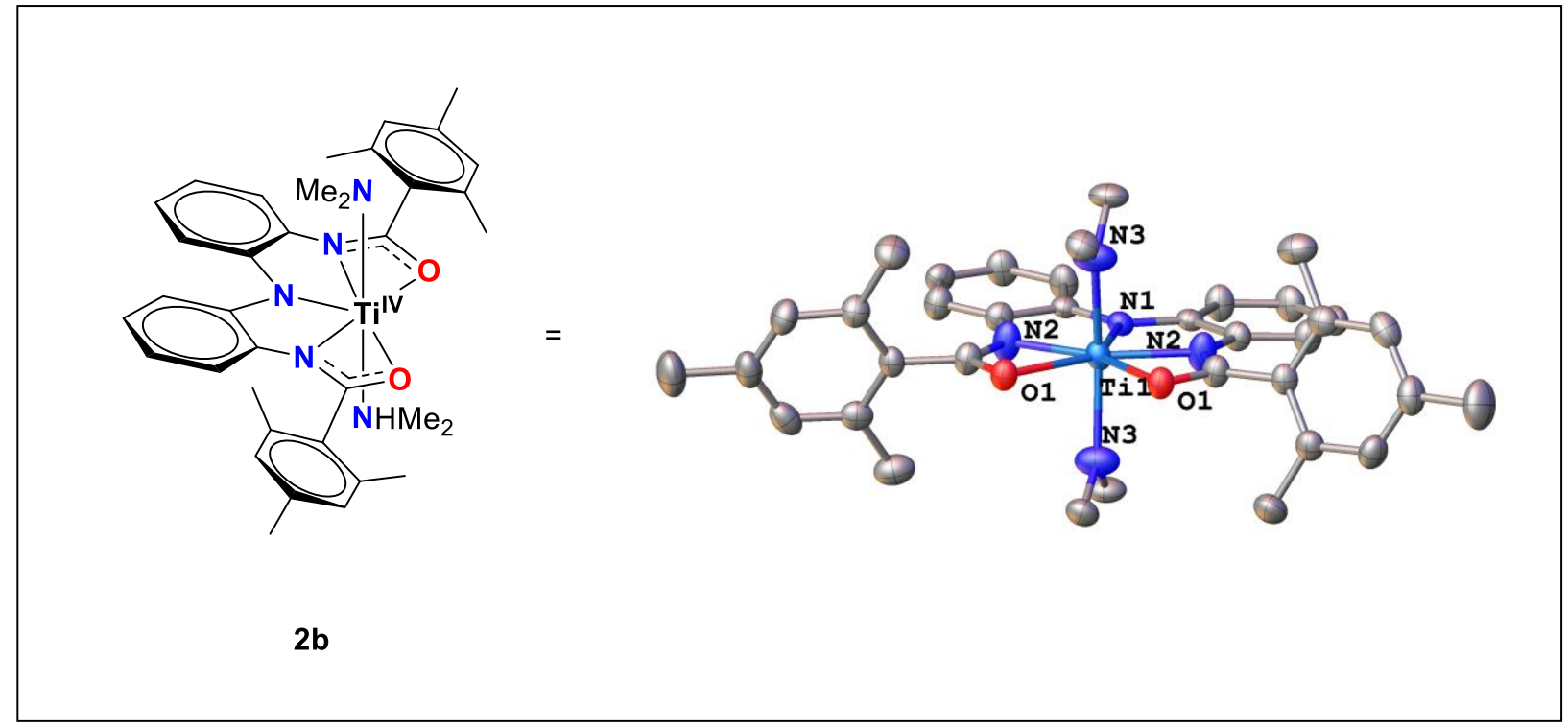

A flask containing $1 \mathbf{b}(500 \mathrm{mg}, 1.02 \mathrm{mmol})$ suspended in diethyl ether $(10 \mathrm{~mL})$ was supplemented with $\mathrm{Ti}\left(\mathrm{NMe}_{2}\right)_{4}(253 \mu \mathrm{L}, 1.07 \mathrm{mmol})$ and stirred until the red solution becomes homogeneous. Standing overnight affords red crystalline material, the supernatant was removed and the solid dried under vacuum. Yield (510 mg, $80 \%$ ). Crystals suitable for XRD were grown from toluene solution at room temperature.

1H-NMR (400 MHz, Benzene- $d_{6}$ ) $\delta 7.89$ (d, 2H, Aryl- $H$ ), 7.07 (ddd, 2H, Aryl- $H$ ), 6.81 (s, 2H, Aryl- $H$ ), 6.76 (s, 2H, Aryl- $H$ ), 6.69 (m, 4H, Aryl- $H$ ), 3.43 (br, 6H, Ti-N(CH3) 2 ), 2.37 (s, 6H, Aryl$\left.\mathrm{CH}_{3}\right), 2.22$ (s, 6H, Aryl-CH3), $2.16(\mathrm{~s}, 6 \mathrm{H}$, Aryl-CH 3$), 2.00\left(\mathrm{~d}, J=6.3 \mathrm{~Hz}, 6 \mathrm{H}, \mathrm{Ti}-\mathrm{NH}\left(\mathrm{CH}_{3}\right)_{2}\right), 1.47$ $\left(\mathrm{m}, 1 \mathrm{H}, \mathrm{Ti}-\mathrm{N} H\left(\mathrm{CH}_{3}\right)_{2}\right)$.

${ }^{13} \mathrm{C}$-NMR $\left(100 \mathrm{MHz}\right.$, Benzene- $\left.d_{6}\right) \delta 175.6(\mathrm{C}=0), 148.3\left(\mathrm{C}_{\mathrm{q}}\right), 139.4\left(\mathrm{C}_{\mathrm{q}}\right), 135.4\left(\mathrm{C}_{\mathrm{q}}\right), 135.2\left(\mathrm{C}_{\mathrm{q}}\right)$, $134.3\left(\mathrm{C}_{\mathrm{q}}\right), 134.2\left(\mathrm{C}_{\mathrm{q}}\right), 129.3($ Aryl $-\mathrm{CH}), 129.0($ Aryl $-\mathrm{CH}), 124.4$ (Aryl- $\left.\mathrm{CH}\right), 120.3$ (Aryl- $\mathrm{CH}$ ), 115.0 (Aryl- $\mathrm{CH}), 113.1$ (Aryl- $\left.\mathrm{CH}), 40.9\left(\text { Ti- } \mathrm{NHCH}_{3}\right)_{2}\right), 21.3$ (Aryl- $\left.\mathrm{CH}_{3}\right), 20.2\left(\right.$ Aryl- $\left.\mathrm{CH}_{3}\right), 19.6$ (Aryl- $-\mathrm{CH}_{3}$ ).

FT-IR (ATR, cm-1): 3316, 3044, 3005, 2971, 2917, 2884, 2851, 2815, 2770, 1612, 1576, $1567,1551,1506,1478,1445,1385,1333,1308,1296,1259,1184,1171,1151,1118,1084$, 1049, 1019, 995, 981, 958, 932, 892, 851, 809, 781, 750, 738, 716, 678, 660, 639, 601, 576, $567,511,493,451,426,405$.

Melting Point: $208^{\circ} \mathrm{C}$ (decomposition).

Elemental Analysis Anal. Found: C, 68.82, H, 6.98; N, 10.94. Calc: C, 69.11; H, 6.93; N, 11.19. 


\section{Compound 2c $\left[\mathrm{C}_{38} \mathrm{H}_{51} \mathrm{~N}_{5} \mathrm{O}_{2} \mathrm{Ti}\right]$}

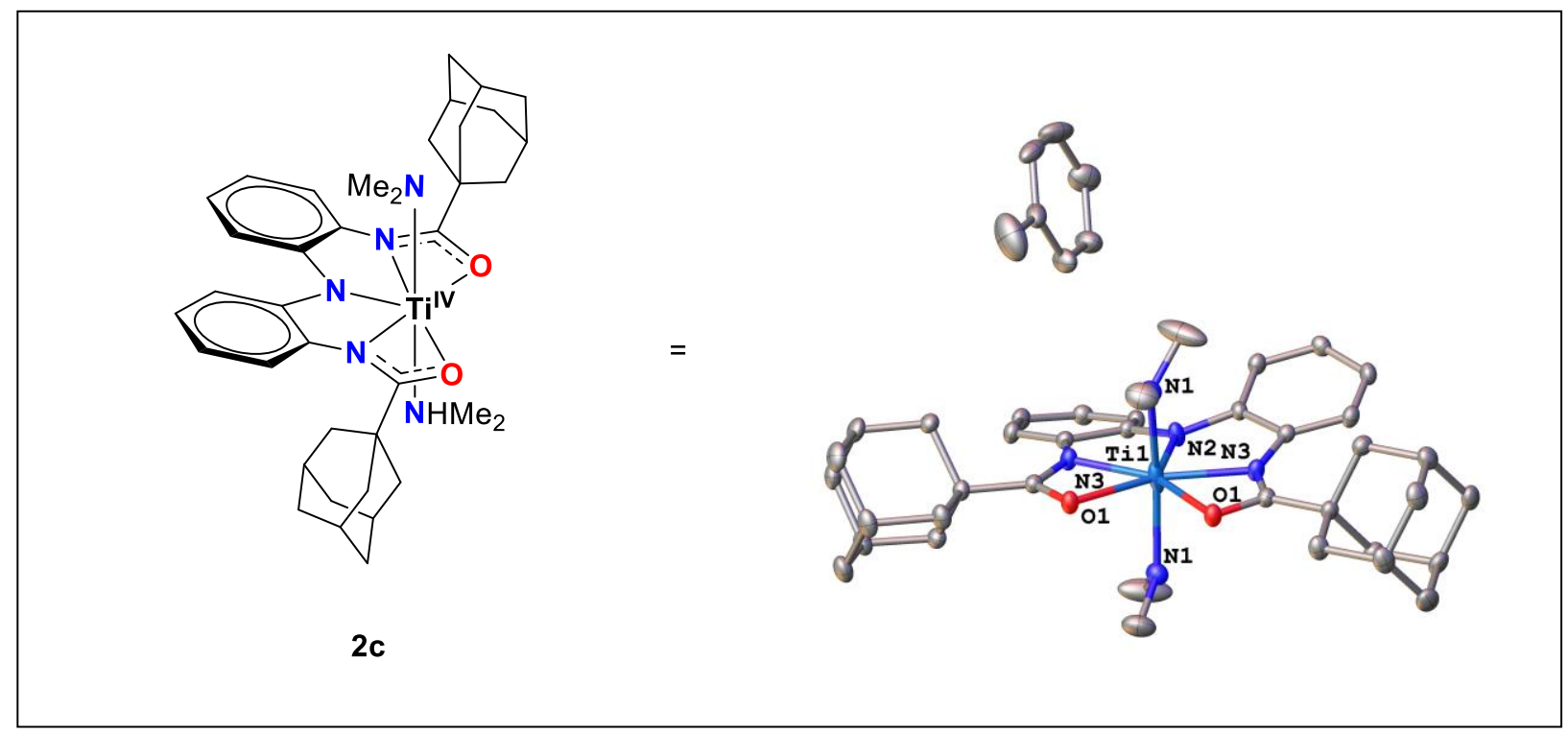

Ligand 1c (350 $\mathrm{mg}, 0.67 \mathrm{mmol}$ ) was suspended in diethyl ether ( $5 \mathrm{~mL}$ ) and followed by the addition of $\mathrm{Ti}\left(\mathrm{NMe}_{2}\right) 4(160 \mu \mathrm{L}, 0.67 \mathrm{mmol})$. After $24 \mathrm{~h}$ of stirring a red powder is obtained over a dark ethereal solution. The ether is discarded and the solid 2c was dried in vacuum (Yield $160 \mathrm{mg}, 36 \%$ ). XRD quality crystals obtained from a saturated toluene solution after cooling to $-25^{\circ} \mathrm{C}$ for a week.

1H-NMR (400 MHz, Benzene- $\left.d_{6}\right) \delta 8.03(\mathrm{dd}, J=8.2,1.3 \mathrm{~Hz}, 2 \mathrm{H}$, Aryl- $H$ ), 7.69 (dd, $J=8.0,1.4$ Hz, 2H, Aryl- $H$ ), 7.20 - $7.11(\mathrm{~m}, 2 \mathrm{H}$, Aryl- $H$ ), 7.04 (ddd, $J=7.9,7.3,1.3 \mathrm{~Hz}, 2 \mathrm{H}$, Aryl- $H$ ), 3.16 (s, 6H, Ti-N(CH3) 2 ), $2.36-2.23\left(\mathrm{~m}, \mathrm{br}, 12 \mathrm{H}\right.$, Adamantyl- $\left.\mathrm{CH}_{2}\right), 1.97$ (p, J = $3.2 \mathrm{~Hz}, 6 \mathrm{H}$, Adamantyl-CH), $1.73-1.60\left(\mathrm{~m}, 18 \mathrm{H}\right.$, Adamantyl- $\left.\mathrm{CH}_{2}+\mathrm{Ti}-\mathrm{NH}\left(\mathrm{CH}_{3}\right)_{2}\right), 0.75$ (br, 1H, Ti$\left.\mathrm{NH}\left(\mathrm{CH}_{3}\right)_{2}\right)$.

${ }^{13}$ C-NMR (100 MHz, Benzene-d6) $\delta 182.8(\mathrm{C}=0), 148.5\left(\mathrm{C}_{\mathrm{q}}\right), 134.9\left(\mathrm{C}_{\mathrm{q}}\right), 123.2$ (Aryl-CH), 120.3 (Aryl- $\mathrm{CH}), 119.4$ (Aryl- $\mathrm{CH}), 113.5($ Aryl- $\mathrm{CH}), 50.5\left(\mathrm{Ti}-\mathrm{N}\left(\mathrm{CH}_{3}\right)_{2}\right), 41.9\left(\mathrm{C}_{\mathrm{q}}\right), 39.8\left(\mathrm{Ti}-\mathrm{NH}\left(\mathrm{CH}_{3}\right)_{2}\right)$, 37.1 (Adamantyl- $\mathrm{CH}_{2}$ ), 36.9 (Adamantyl- $\mathrm{CH}_{2}$ ), 28.5 (Adamantyl- $\mathrm{CH}$ ).

FT-IR (ATR, cm-1): 3300, 2904, 2847, 2758, 1652, 1570, 1525, 1249, 1389, 1364, 1342, 1298, $1258,1237,1212,1180,1120,1101,1077,1035,981,958,943,934,890,855,832,812,772$, $747,734,716,709,684,646,594,574,522,492,478,451$.

Melting Point: $177^{\circ} \mathrm{C}$ (decomposition).

Elemental Analysis [ $\left.\mathrm{C}_{38} \mathrm{H}_{51} \mathrm{~N}_{5} \mathrm{O}_{2} \mathrm{Ti} \cdot \mathrm{C}_{7} \mathrm{H}_{8}\right]$ Anal. Found: C, 72.36; H, 7.84; N 10.55. Calc: C, 72.08; H, 7.93; N, 9.34. 


\section{Compound 2d [ $\left.\mathrm{C}_{48} \mathrm{H}_{67} \mathrm{~N}_{5} \mathrm{O}_{2} \mathrm{Ti}\right]$}

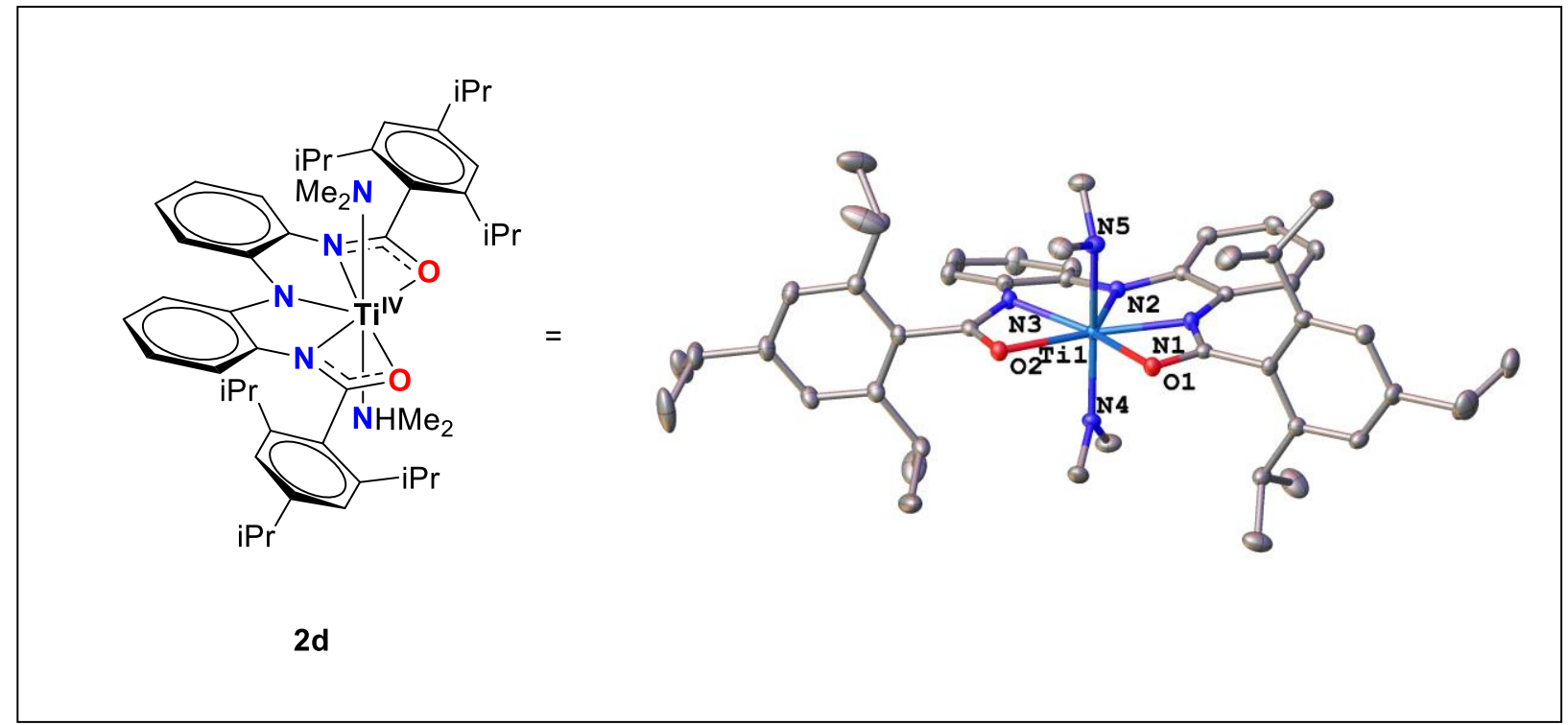

A solution of $\mathbf{1 d}$ ( $400 \mathrm{mg}, 0.61 \mathrm{mmol})$ in toluene $\left(10 \mathrm{~mL}\right.$ ) was supplemented with $\mathrm{Ti}\left(\mathrm{NMe}_{2}\right) 4$ (145 $\mu \mathrm{L}, 0.61 \mathrm{mmol}$ ) in toluene $(5 \mathrm{~mL})$. The solution was stirred overnight and concentrated until the red product started to precipitate. Hexane was cannula transferred $(20 \mathrm{~mL})$ and causing more solid to precipitate. The blood red powdery product was isolated by cannula filtration. Yield (259 mg, $54 \%$ ). Single crystals were obtained from a saturated toluene solution at room temperature.

${ }^{1}$ H-NMR (400 MHz, Benzene- $\left.d_{6}\right) \delta 7.82(\mathrm{dd}, J=8.3,1.1 \mathrm{~Hz}, 2 \mathrm{H}$, Aryl- $H$ ), $7.28(\mathrm{~s}, 4 \mathrm{H}$, Aryl- $H$ ), 6.98 (ddd, $J=8.5,7.0,1.9 \mathrm{~Hz}, 2 \mathrm{H}$, Aryl- $H$ ), $6.62-6.50$ (m, 4H, Aryl- $H$ ), 3.73 (br, 3H, Ti$\left.\mathrm{N}\left(\mathrm{CH}_{3}\right)_{2}\right), 3.33$ (p, J = 6.9 Hz, 2H, Aryl-CH(CH$\left.)_{2}\right), 3.23\left(\mathrm{br}, 3 \mathrm{H}, \mathrm{Ti}-\mathrm{N}\left(\mathrm{CH}_{3}\right)_{2}\right), 3.11$ (p, J = $6.8 \mathrm{~Hz}$, $2 \mathrm{H}$, Aryl- $\left.\mathrm{CH}\left(\mathrm{CH}_{3}\right)_{2}\right), 2.88\left(\mathrm{p}, J=6.9 \mathrm{~Hz}, 2 \mathrm{H}, \operatorname{Aryl}-\mathrm{CH}\left(\mathrm{CH}_{3}\right)_{2}\right), 2.16(\mathrm{~d}, J=6.3 \mathrm{~Hz}, 6 \mathrm{H}$, Ti$\left.\mathrm{NH}\left(\mathrm{CH}_{3}\right)_{2}\right), 1.77\left(\mathrm{br}, 1 \mathrm{H}, \mathrm{Ti}-\mathrm{N} H\left(\mathrm{CH}_{3}\right)_{2}\right), 1.34\left(\mathrm{~d}, J=6.8 \mathrm{~Hz}, 6 \mathrm{H}, \operatorname{Aryl}-\mathrm{CH}\left(\mathrm{CH}_{3}\right)_{2}\right), 1.31-1.28(\mathrm{~m}$, 24H, Aryl-CH(CH3)2), $1.15\left(\mathrm{~d}, \mathrm{~J}=6.7 \mathrm{~Hz}, 6 \mathrm{H}\right.$, Aryl- $\left.\mathrm{CH}\left(\mathrm{CH}_{3}\right)_{2}\right)$.

${ }^{13} \mathrm{C}$-NMR $\left(100 \mathrm{MHz}\right.$, Benzene- $\left.d_{6}\right) \delta 176.6(\mathrm{C}=0), 151.0\left(\mathrm{C}_{\mathrm{q}}\right), 148.2\left(\mathrm{C}_{\mathrm{q}}\right), 146.5\left(\mathrm{C}_{\mathrm{q}}\right), 144.9\left(\mathrm{C}_{\mathrm{q}}\right)$, $135.5\left(\mathrm{C}_{\mathrm{q}}\right), 133.5\left(\mathrm{C}_{\mathrm{q}}\right), 124.2($ Aryl $-\mathrm{CH}), 122.1($ Aryl $-\mathrm{CH}), 121.3($ Aryl- $\mathrm{CH}), 120.0($ Aryl $-\mathrm{CH})$, 115.6 (Aryl- $\mathrm{CH}), 113.1$ (Aryl- $\mathrm{CH}), 40.8$ (Ti- $\left.\mathrm{NH}\left(\mathrm{CH}_{3}\right)_{2}\right), 34.8$ (Aryl- $\left.\mathrm{CH}\left(\mathrm{CH}_{3}\right)_{2}\right), 32.1$ (Aryl$\left.\mathrm{CH}\left(\mathrm{CH}_{3}\right)_{2}\right), 31.5$ (Aryl- $\left.\mathrm{CH}\left(\mathrm{CH}_{3}\right)_{2}\right), 25.8\left(\mathrm{Aryl}-\mathrm{CH}\left(\mathrm{CH}_{3}\right)_{2}\right), 24.8\left(\mathrm{Aryl}-\mathrm{CH}\left(\mathrm{CH}_{3}\right)_{2}\right), 24.5$ (Aryl$\left.\mathrm{CH}\left(\mathrm{CH}_{3}\right) 2\right), 24.2$ (Aryl-CH$\left.\left(\mathrm{CH}_{3}\right)_{2}\right), 23.9$ (Aryl-CH$\left.\left(\mathrm{CH}_{3}\right) 2\right)$.

FT-IR (ATR, cm-1) 3290, 3020, 2960, 2865, 2812, 2769, 1608, 1574, 1541, 1479, 1450, 1425, $1404,1395,1362,1382,1317,1265,1242,1178,1143,1116,1103,1051,1081,1031,988$, $947,934,911,889,877,822,797,758,744,730,718,686,655,609,576,565,528,484,409$.

Melting Point: $221^{\circ} \mathrm{C}$ (decomposition).

Elemental Analysis Anal. Found: C, 72.41; H, 7.90; N, 8.60. Calc: C, 72.61; H, 8.51; N, 8.82. 


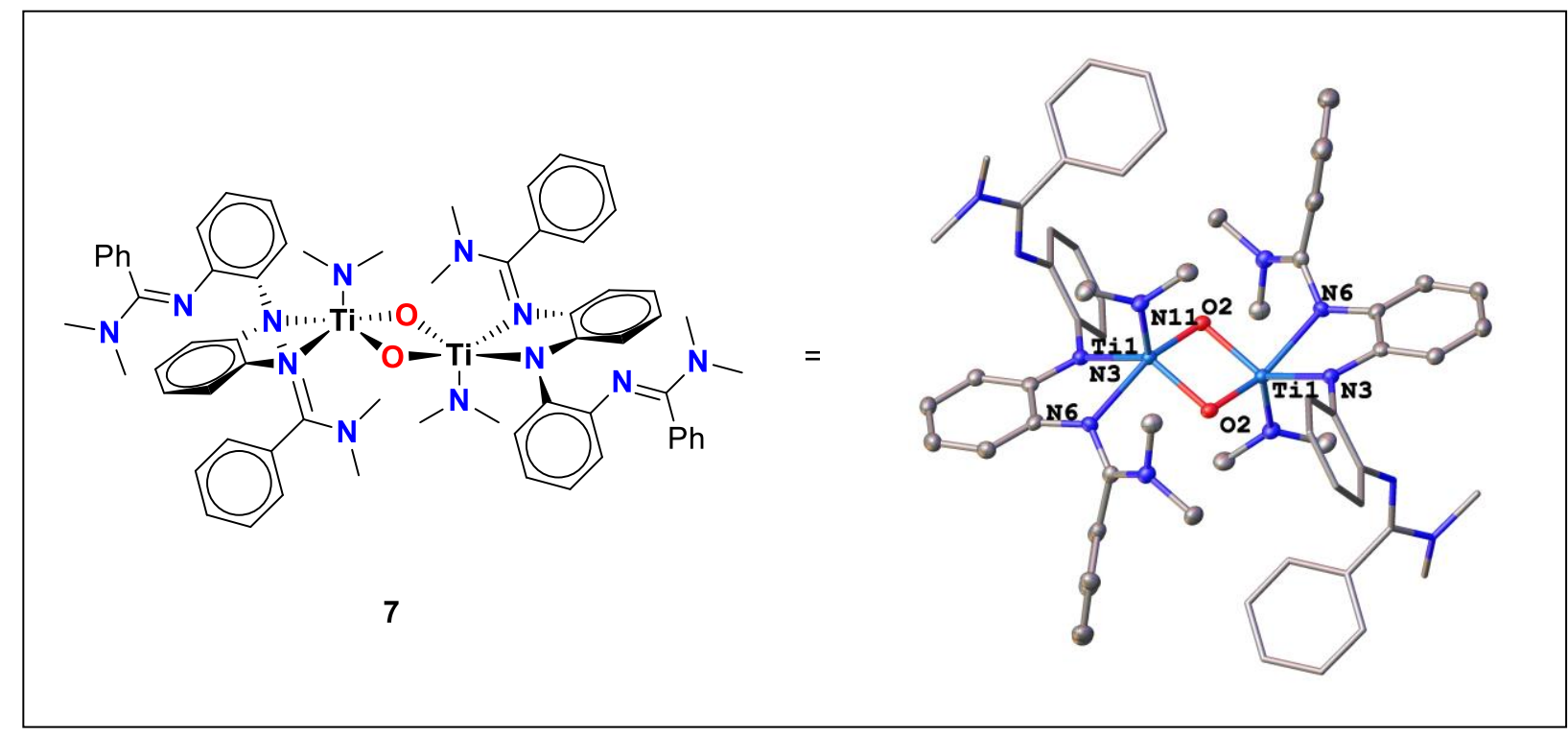

A vial containing (100 mg, $0.25 \mathrm{mmol})$ of $1 \mathbf{e}$ suspended in toluene (1.5 mL) was supplemented with $\mathrm{Ti}\left(\mathrm{NMe}_{2}\right)_{4}(64 \mu \mathrm{L}, 0.27 \mathrm{mmol})$. The mixture was stirred for $24 \mathrm{~h}$ at room temperature and then cooled to $-25{ }^{\circ} \mathrm{C}$ for a week afforded a brown gooey precipitate containing a fine crystalline residue. The precipitate was transferred to a microscope slide with paratone oil from where a red XRD quality crystal was retrieved. 


\section{Compound 3d [ $\left.\mathrm{C}_{48} \mathrm{H}_{67} \mathrm{~N}_{5} \mathrm{O}_{2} \mathrm{Zr}\right]$}

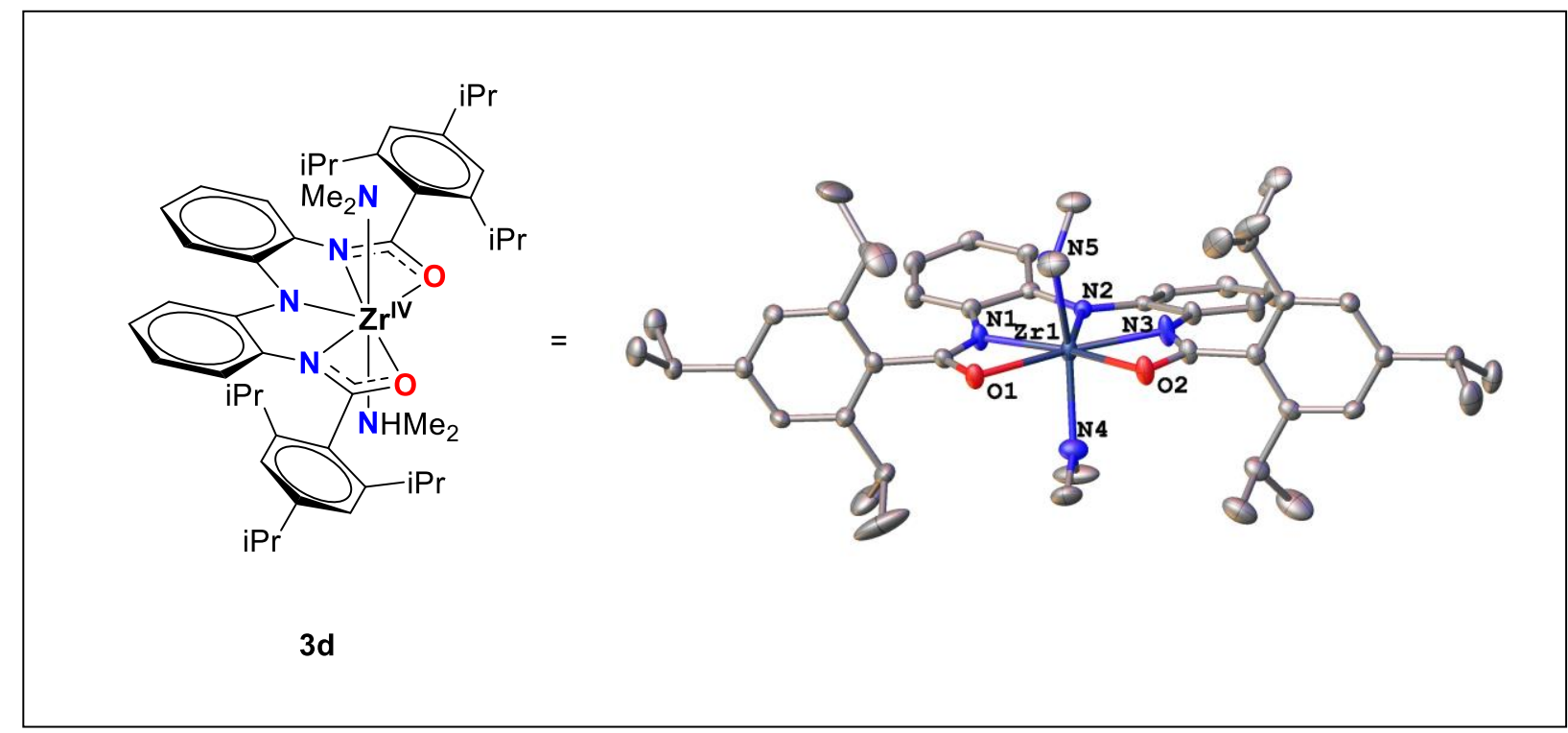

Ligand 1d (317 mg, $0.48 \mathrm{mmol}$ ) was dissolved in toluene ( $3 \mathrm{~mL}$ ) followed by the addition of solid $\mathrm{Zr}\left(\mathrm{NMe}_{2}\right) 4(130 \mathrm{mg}, 0.48 \mathrm{mmol})$. After stirring for 2 hours at room temperature hexanes $(20 \mathrm{~mL})$ were added and yellow suspension was obtained. The supernatant was removed and the solid was dried in vacuum to afford the title compound as a light-yellow solid. Yield (185 mg, $46 \%$ ). Single crystals were grown from saturated toluene solution.

${ }^{1}$ H-NMR (400 MHz, Benzene- $\left.d_{6}\right) \delta 7.95$ (dd, $J=8.3,1.1 \mathrm{~Hz}, 2 \mathrm{H}, \mathrm{Ar}-H$ ), $7.28(\mathrm{~s}, 4 \mathrm{H}, \mathrm{Ar}-H$ ), $6.94(\mathrm{~m}, 2 \mathrm{H}, \mathrm{Ar}-H), 6.60$ (dd, $J=7.8,1.6 \mathrm{~Hz}, 2 \mathrm{H}, \mathrm{Ar}-H), 6.48(\mathrm{~m}, 2 \mathrm{H}, \mathrm{Ar}-H), 3.27$ (hept, $J=7.1$ $\left.\mathrm{Hz}, 4 \mathrm{H}, \mathrm{Ar}-\mathrm{CH}\left(\mathrm{CH}_{3}\right)_{2}\right), 3.20$ (s, 6H, Zr-N(CH3)2), 2.87 (hept, $\left.J=6.9 \mathrm{~Hz}, 2 \mathrm{H}, \mathrm{Ar}-\mathrm{CH}\left(\mathrm{CH}_{3}\right)_{2}\right), 2.20$ (s, 6H, Zr-NH(CH3)2), 1.96 (br, $\left.1 \mathrm{H}, \mathrm{Zr}-\mathrm{NH}\left(\mathrm{CH}_{3}\right)_{2}\right), 1.37$ - 1.31 (m, 18H, $\left.\mathrm{Ar}-\mathrm{CH}\left(\mathrm{CH}_{3}\right)_{2}\right), 1.28$ $\left(\mathrm{dd}, J=6.9,2.2 \mathrm{~Hz}, 12 \mathrm{H}, \mathrm{Ar}-\mathrm{CH}\left(\mathrm{CH}_{3}\right)_{2}\right), 1.19-1.16\left(\mathrm{~d}, J=6.8 \mathrm{~Hz}, 6 \mathrm{H}, \mathrm{Ar}-\mathrm{CH}\left(\mathrm{CH}_{3}\right)_{2}\right)$.

${ }^{13}$ C-NMR $\left(100 \mathrm{MHz}\right.$, Benzene- $\left.d_{6}\right) \delta 179.31(C=0), 151.0\left(\mathrm{C}_{\mathrm{q}}\right), 148.3\left(\mathrm{C}_{\mathrm{q}}\right), 146.2\left(\mathrm{C}_{\mathrm{q}}\right), 144.4$ $\left(\mathrm{C}_{\mathrm{q}}\right), 135.1\left(\mathrm{C}_{\mathrm{q}}\right), 133.5\left(\mathrm{C}_{\mathrm{q}}\right), 124.5(\mathrm{Ar}-\mathrm{CH}), 122.2(\mathrm{Ar}-\mathrm{CH}), 121.2(\mathrm{Ar}-\mathrm{CH}), 118.9(\mathrm{Ar}-\mathrm{CH})$, 116.6 (Ar- $\mathrm{CH}), 115.0(\mathrm{Ar}-\mathrm{CH}), 44.2\left(\mathrm{Ti}-\mathrm{N}\left(\mathrm{CH}_{3}\right)_{2}\right), 39.7\left(\mathrm{br}, \mathrm{Ti}-\mathrm{NH}\left(\mathrm{CH}_{3}\right)_{2}\right), 34.9\left(\mathrm{Ar}-\mathrm{CH}\left(\mathrm{CH}_{3}\right)_{2}\right)$, $32.3\left(\mathrm{Ar}-\mathrm{CH}\left(\mathrm{CH}_{3}\right)_{2}\right), 31.7\left(\mathrm{Ar}-\mathrm{CH}\left(\mathrm{CH}_{3}\right)_{2}\right), 25.8\left(\mathrm{Ar}-\mathrm{CH}\left(\mathrm{CH}_{3}\right)_{2}\right), 25.2\left(\mathrm{Ar}-\mathrm{CH}\left(\mathrm{CH}_{3}\right)_{2}\right), 24.6(\mathrm{Ar}-$ $\left.\mathrm{CH}\left(\mathrm{CH}_{3}\right)_{2}\right), 24.3\left(\mathrm{Ar}-\mathrm{CH}\left(\mathrm{CH}_{3}\right)_{2}\right), 23.5\left(\mathrm{Ar}-\mathrm{CH}\left(\mathrm{CH}_{3}\right)_{2}\right)$.

FT-IR (ATR, cm-1) 3307, 3054, 2958, 2867, 1607, 1574, 1561, 1529, 1477, 1449, 1416, $1401,1382,1361,1298,1260,1180,1153,1143,1115,1102,1079,1054,1033,990,946$, $933,886,876,821,796,758,744,734,716,681,653,609,564,541,517,483,452,427$.

Melting Point: $231-232^{\circ} \mathrm{C}$

Elemental Analysis Anal. Found: C, 68.68; H, 7.87; N, 8.38. Calc. $\left(\mathrm{C}_{48} \mathrm{H}_{67} \mathrm{~N}_{5} \mathrm{O}_{3} \mathrm{Zr}\right): \mathrm{C}, 68.85$; H, 8.07; N, 8.36. 


\section{Compound 4d [ $\left.\mathrm{C}_{48} \mathrm{H}_{67} \mathrm{~N}_{5} \mathrm{O}_{2} \mathrm{Hf}\right]$}

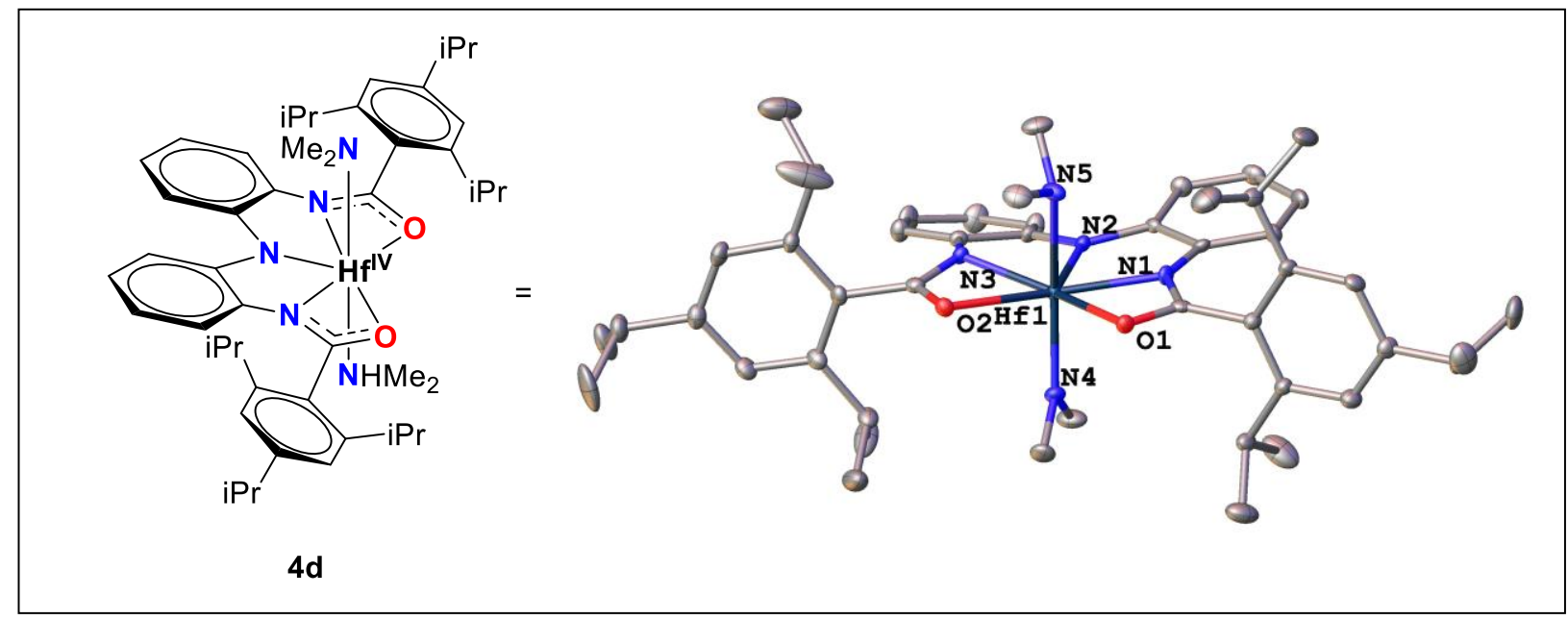

Ligand $1 \mathbf{d}$ (300 $\mathrm{mg}, 0.45 \mathrm{mmol})$ was dissolved in toluene $(1.2 \mathrm{~mL})$ followed by the addition of solid $\mathrm{Hf}\left(\mathrm{NMe}_{2}\right) 4(180 \mathrm{mg}, 0.51 \mathrm{mmol})$. After stirring for $24 \mathrm{~h}$ hours at room temperature a yellow microcrystalline residue forms over a deep yellow solution. Hexanes were added (10 $\mathrm{mL}$ ) causing more product to precipitate out of solution. The supernatant was removed, and the light-yellow powder was dried under vacuum. (242 mg, $58 \%$ ). XRD quality specimens were obtained from concentrated toluene solution at room temperature.

${ }^{1}$ H-NMR (400 MHz, Benzene- $\left.d_{6}\right) \delta 7.96(\mathrm{dd}, J=8.3,1.1 \mathrm{~Hz}, 2 \mathrm{H}, \mathrm{Ar}-H), 7.29-7.26(\mathrm{~m}, 4 \mathrm{H}, \mathrm{Ar}-$ $H$ ), 6.98-6.94 (m, 2H, Ar- $H$ ), $6.62(\mathrm{dd}, J=7.8,1.6 \mathrm{~Hz}, 2 \mathrm{H}, \mathrm{Ar}-H), 6.46(\mathrm{td}, J=7.6,1.1 \mathrm{~Hz}, 2 \mathrm{H}, \mathrm{Ar}-$ $H$ ), 3.35 - $3.24\left(\mathrm{~m}, 10 \mathrm{H}, \mathrm{Ar}-\mathrm{CH}\left(\mathrm{CH}_{3}\right)_{2}+\mathrm{Hf}-\mathrm{N}\left(\mathrm{CH}_{3}\right)_{2}\right), 2.87$ (hept, $\left.J=6.9 \mathrm{~Hz}, 2 \mathrm{H}, \mathrm{Ar}-\mathrm{CH}\left(\mathrm{CH}_{3}\right)_{2}\right)$, $2.21\left(\mathrm{~d}, J=6.2 \mathrm{~Hz}, 6 \mathrm{H}, \mathrm{Hf}-\mathrm{NH}\left(\mathrm{CH}_{3}\right)_{2}\right), 1.97$ (br, $\left.1 \mathrm{H}, \mathrm{Hf}-\mathrm{NH}\left(\mathrm{CH}_{3}\right)_{2}\right), 1.37$ (d, $J=6.7 \mathrm{~Hz}, 6 \mathrm{H}, \mathrm{Ar}-$ $\left.\mathrm{CH}\left(\mathrm{CH}_{3}\right)_{2}\right), 1.33\left(\mathrm{~m}, 12 \mathrm{H}, \mathrm{Ar}-\mathrm{CH}\left(\mathrm{CH}_{3}\right)_{2}\right), 1.28\left(\mathrm{dd}, J=6.9,2.1 \mathrm{~Hz}, 12 \mathrm{H}, \mathrm{Ar}-\mathrm{CH}\left(\mathrm{CH}_{3}\right)_{2}\right), 1.21-$ $1.18\left(\mathrm{~d}, 6 \mathrm{H}, \mathrm{Ar}-\mathrm{CH}\left(\mathrm{CH}_{3}\right)_{2}\right)$.

${ }^{13}$ C-NMR $\left(101 \mathrm{MHz}\right.$, Benzene- $\left.d_{6}\right) \delta 178.7(C=0), 151.0\left(\mathrm{C}_{\mathrm{q}}\right), 148.1\left(\mathrm{C}_{\mathrm{q}}\right), 146.6\left(\mathrm{C}_{\mathrm{q}}\right), 144.4\left(\mathrm{C}_{\mathrm{q}}\right)$, 134.8 $\left(\mathrm{C}_{\mathrm{q}}\right), 133.6\left(\mathrm{C}_{\mathrm{q}}\right), 124.8(\mathrm{Ar}-\mathrm{CH}), 122.3(\mathrm{Ar}-\mathrm{CH}), 121.1(\mathrm{Ar}-\mathrm{CH}), 118.7(\mathrm{Ar}-\mathrm{CH}), 117.0(\mathrm{Ar}-$ $\mathrm{CH}), 115.7(\mathrm{Ar}-\mathrm{CH}), 44.0\left(\mathrm{Ti}-\mathrm{N}\left(\mathrm{CH}_{3}\right)_{2}\right), 39.8\left(\mathrm{Ti}-\mathrm{NH}\left(\mathrm{CH}_{3}\right)_{2}\right), 34.9\left(\mathrm{Ar}-\mathrm{CH}\left(\mathrm{CH}_{3}\right)_{2}\right), 32.3(\mathrm{Ar}-$ $\left.\mathrm{CH}\left(\mathrm{CH}_{3}\right)_{2}\right), 31.6\left(\mathrm{Ar}-\mathrm{CH}\left(\mathrm{CH}_{3}\right)_{2}\right), 25.8\left(\mathrm{Ar}-\mathrm{CH}\left(\mathrm{CH}_{3}\right)_{2}\right), 25.1\left(\mathrm{Ar}-\mathrm{CH}\left(\mathrm{CH}_{3}\right)_{2}\right), 24.7\left(\mathrm{Ar}-\mathrm{CH}\left(\mathrm{CH}_{3}\right)_{2}\right)$, $24.2\left(\mathrm{Ar}-\mathrm{CH}\left(\mathrm{CH}_{3}\right)_{2}\right), 23.5\left(\mathrm{Ar}-\mathrm{CH}\left(\mathrm{CH}_{3}\right)_{2}\right)$.

FT-IR (ATR, cm-1) 3299, 2959, 2867, 2775, 1607, 1573, 1535, 1478, 1450, 1400, 1383, 1362, 1296, 1256, 1180, 1143, 1115, 1102, 1079, 1064, 1033, 988, 947, 934, 888, 876, 829, 796, $758,731,718,681,654,609,569,483,454,428$.

Melting Point: $243^{\circ} \mathrm{C}$.

Elemental Analysis Anal. Found: C, 61.91; H, 6.90; N, 7.22. Calc. $\left(\mathrm{C}_{48} \mathrm{H}_{67} \mathrm{~N}_{5} \mathrm{O}_{3} \mathrm{Hf}\right): \mathrm{C}, 62.36 ; \mathrm{H}$, $7.30 ; \mathrm{N}, 7.56$. 


\section{Compound 5d [ $\left.\mathrm{C}_{48} \mathrm{H}_{67} \mathrm{~N}_{5} \mathrm{O}_{2} \mathrm{Nb}\right]$}

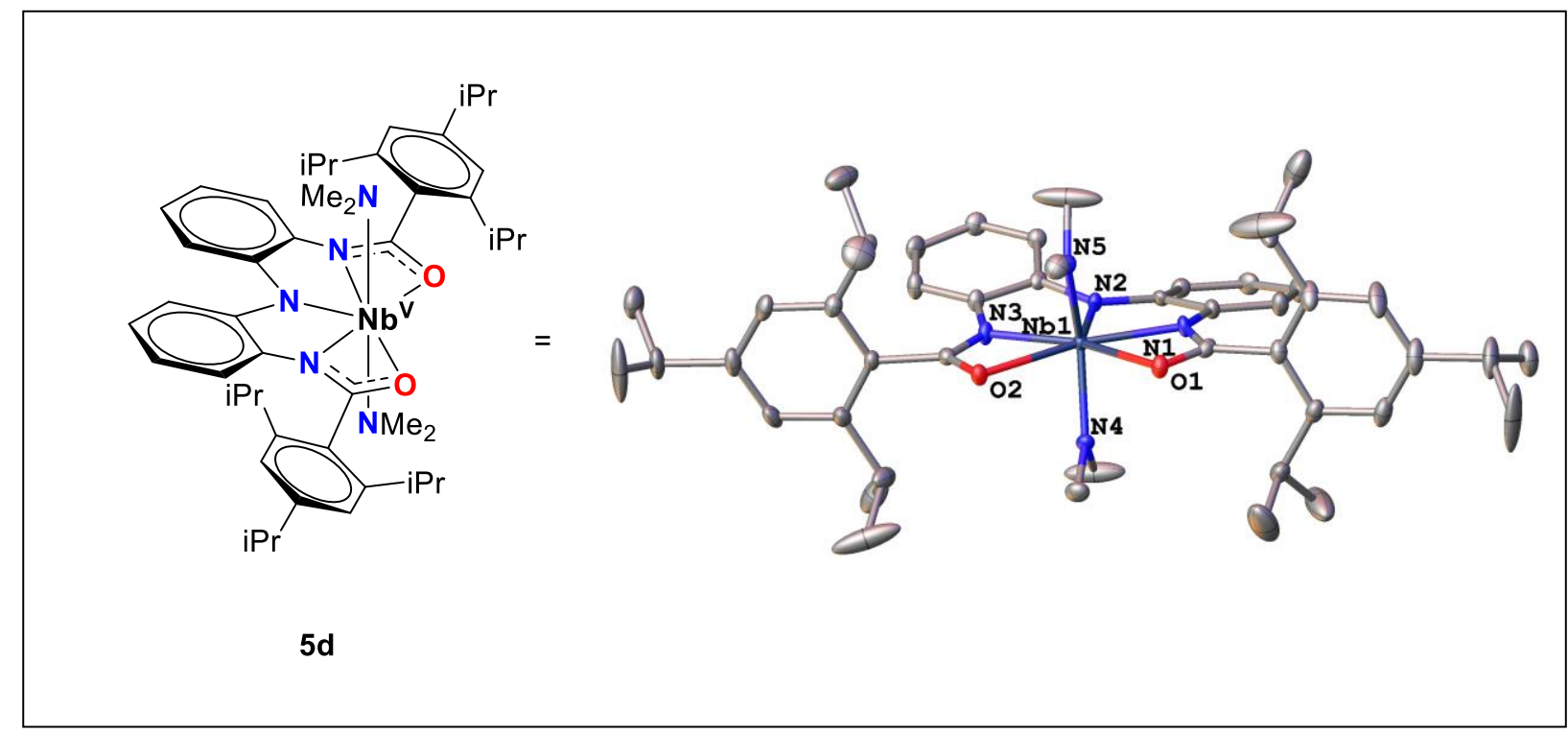

A vial was charged with ligand $\mathbf{1 d}$ ( $200 \mathrm{mg}, 0.303 \mathrm{mmol}$ ) and suspended in toluene $(1.5 \mathrm{~mL})$. Solid $\mathrm{Nb}\left(\mathrm{NMe}_{2}\right) 5(100 \mathrm{mg}, 0.318 \mathrm{mmol})$ was added turning the solution black. The mixture was stirred until homogeneous. Crystalline precipitate formed after a few hours. The supernatant was removed, and the yellow solid was washed with diethyl ether ( $6 \times 1 \mathrm{~mL})$ before being dried under vacuum. Yield (94 mg, $37 \%$ ).

${ }^{1}$ H-NMR (400 MHz, Methylene Chloride- $d_{2}$ ) $\delta 7.72(\mathrm{dd}, J=8.3,1.1 \mathrm{~Hz}, 2 \mathrm{H}, \mathrm{Ar}-H), 7.16(\mathrm{~s}, 4 \mathrm{H}$, Ar- $H$ ), 6.96 (ddd, $J=8.5,7.3,1.5 \mathrm{~Hz}, 2 \mathrm{H}, \mathrm{Ar}-H$ ), 6.62 (ddd, $J=8.3,7.3,1.1 \mathrm{~Hz}, 2 \mathrm{H}, \mathrm{Ar}-H$ ), 6.14 (dd, $J=8.0,1.4 \mathrm{~Hz}, 2 \mathrm{H}, \mathrm{Ar}-\mathrm{H}), 3.17\left(\mathrm{br}, 12 \mathrm{H}, \mathrm{Nb}-\mathrm{N}\left(\mathrm{CH}_{3}\right)_{2}\right), 2.96\left(\mathrm{~m}, 6 \mathrm{H}, \mathrm{Ar}-\mathrm{CH}\left(\mathrm{CH}_{3}\right)_{2}\right), 1.33(\mathrm{~d}$, $\left.J=6.9 \mathrm{~Hz}, 12 \mathrm{H}, \mathrm{Ar}-\mathrm{CH}\left(\mathrm{CH}_{3}\right)_{2}\right), 1.26\left(\mathrm{~d}, J=6.8 \mathrm{~Hz}, 12 \mathrm{H}, \mathrm{Ar}-\mathrm{CH}\left(\mathrm{CH}_{3}\right)_{2}\right), 1.11(\mathrm{~d}, J=6.8 \mathrm{~Hz}, 12 \mathrm{H}$, $\left.\mathrm{Ar}-\mathrm{CH}\left(\mathrm{CH}_{3}\right)_{2}\right)$.

${ }^{13}$ C-NMR (101 MHz, Methylene Chloride- $\left.d_{2}\right) \delta 177.5(C=0), 151.5\left(\mathrm{C}_{\mathrm{q}}\right), 146.3\left(\mathrm{C}_{\mathrm{q}}\right), 145.2\left(\mathrm{C}_{\mathrm{q}}\right)$, $136.3\left(\mathrm{C}_{\mathrm{q}}\right), 133.4\left(\mathrm{C}_{\mathrm{q}}\right), 123.0(\mathrm{Ar}-\mathrm{CH}), 121.7(\mathrm{Ar}-\mathrm{CH}), 121.1(\mathrm{Ar}-\mathrm{CH}), 115.4(\mathrm{Ar}-\mathrm{CH}), 114.9(\mathrm{Ar}-$ $\mathrm{CH}), 47.1\left(\mathrm{br}, \mathrm{Nb}-\mathrm{N}\left(\mathrm{CH}_{3}\right)_{2}\right) 35.0\left(\mathrm{Ar}-\mathrm{CH}\left(\mathrm{CH}_{3}\right)_{2}\right), 31.6\left(\mathrm{Ar}-\mathrm{CH}\left(\mathrm{CH}_{3}\right)_{2}\right), 25.1\left(\mathrm{Ar}-\mathrm{CH}\left(\mathrm{CH}_{3}\right)_{2}\right), 24.2$ $\left(\mathrm{Ar}-\mathrm{CH}\left(\mathrm{CH}_{3}\right)_{2}\right), 23.8\left(\mathrm{Ar}-\mathrm{CH}\left(\mathrm{CH}_{3}\right)_{2}\right)$.

FT-IR (ATR, cm-1) 2956, 2864, 2777, 1606, 1567, 1525, 1526, 1511, 1485, 1450, 1436, 1395 , $1381,1361,1315,1256,1178,1153,1143,1117,1101,1080,1053,1033,937,895,801$, $876,834,757,731,716,684,652,609,571,561,520,475$.

Melting Point: $259-260{ }^{\circ} \mathrm{C}$ (decomposition).

Elemental Analysis Anal. Found: C, 68.16; H, 7.52; N, 7.77. Calc. $\left(\mathrm{C}_{48} \mathrm{H}_{66} \mathrm{~N}_{5} \mathrm{O}_{3} \mathrm{Nb}\right): \mathrm{C}, 68.80$; H, 7.94; N, 8.36. 


\section{Compound 6d [ $\left.\mathrm{C}_{48} \mathrm{H}_{67} \mathrm{~N}_{5} \mathrm{O}_{2} \mathrm{Ta}\right]$}

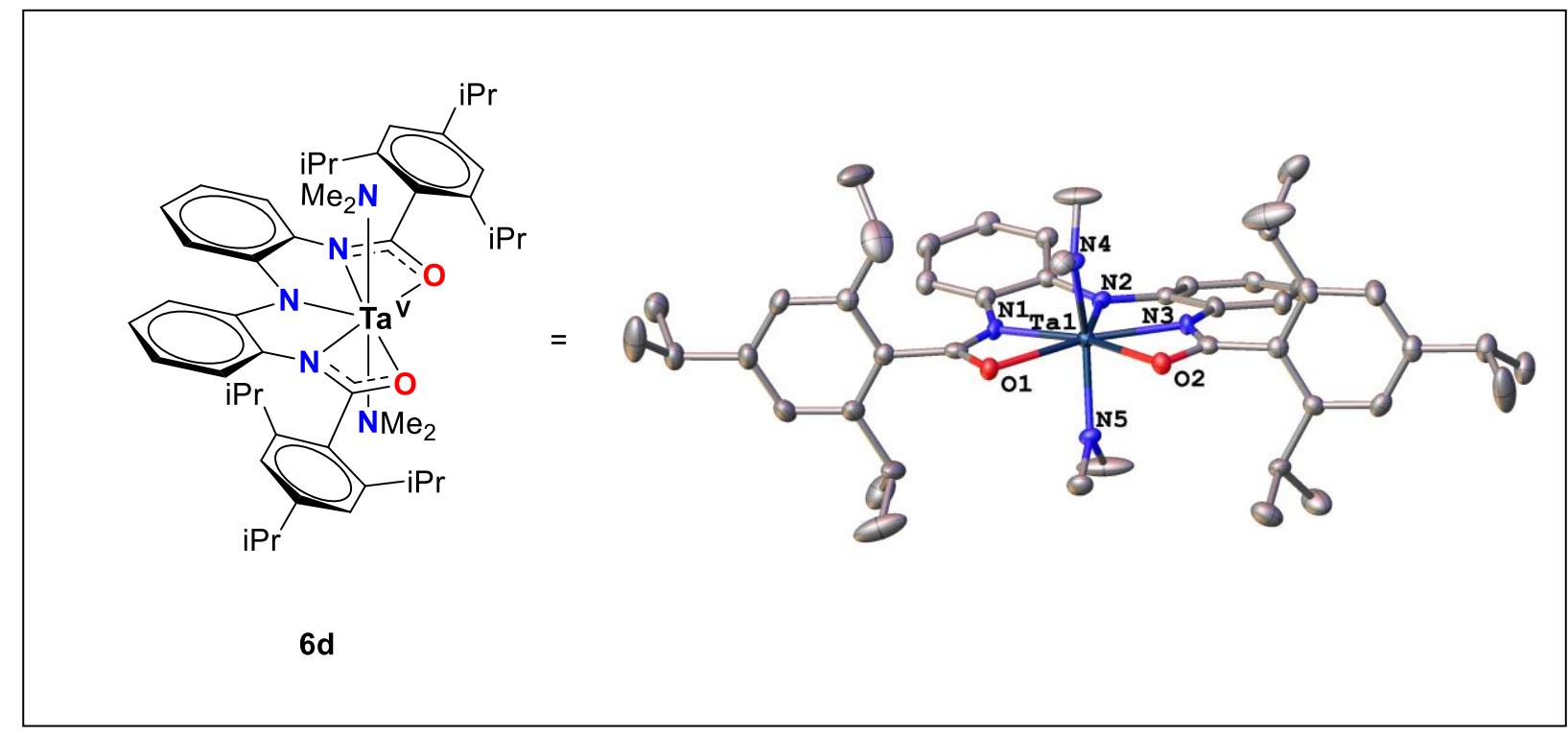

A vial was charged with ligand ( $275 \mathrm{mg}, 0.42 \mathrm{mmol}$ ) and was suspended in toluene ( $2 \mathrm{~mL}$ ). Solid $\mathrm{Ta}\left(\mathrm{NMe}_{2}\right)_{5}$ was added (184 $\mathrm{mg}, 0.46 \mathrm{mmol}$ ) and the vial was stirred until an homogeneous orange solution formed. The solution was allowed to stand until visible precipitate forms (after $\sim 2$ hours). Hexanes $(10 \mathrm{~mL}$ ) was added and the mixture was shaken. The orange supernatant was discarded, the yellow solid was washed with hexanes (2 mL) and dried under vacuum. Yield (227 mg, Yield $59 \%$ ). Single crystals were grown from concentrated toluene solution.

1H-NMR (400 MHz, Methylene Chloride- $d_{2}$ ) $\delta 7.76(\mathrm{dd}, J=8.3,1.2 \mathrm{~Hz}, 2 \mathrm{H}, \mathrm{Ar}-H$ ), 7.00 (ddd, $J$ $=8.5,7.3,1.5 \mathrm{~Hz}, 2 \mathrm{H}, \mathrm{Ar}-H), 6.63(\mathrm{ddd}, J=8.2,7.3,1.1 \mathrm{~Hz}, 2 \mathrm{H}, \mathrm{Ar}-H), 6.18(\mathrm{dd}, J=7.9,1.4 \mathrm{~Hz}$, 2H, Ar- $H$ ) , $3.18\left(\mathrm{br}, 12 \mathrm{H}, \mathrm{Ta}-\mathrm{N}\left(\mathrm{CH}_{3}\right)_{2}\right) 2.97\left(\mathrm{~m}, 6 \mathrm{H}, \mathrm{Ar}-\mathrm{CH}\left(\mathrm{CH}_{3}\right)_{2}\right), 1.33(\mathrm{~d}, J=6.9 \mathrm{~Hz}, 12 \mathrm{H}, \mathrm{Ar}-$ $\left.\mathrm{CH}\left(\mathrm{CH}_{3}\right)_{2}\right), 1.27\left(\mathrm{~d}, J=6.8 \mathrm{~Hz}, 12 \mathrm{H}, \mathrm{Ar}-\mathrm{CH}\left(\mathrm{CH}_{3}\right)_{2}\right), 1.11\left(\mathrm{~d}, J=6.9 \mathrm{~Hz}, 12 \mathrm{H}, \mathrm{Ar}-\mathrm{CH}\left(\mathrm{CH}_{3}\right)_{2}\right)$.

${ }^{13}$ C-NMR (101 MHz, Methylene Chloride- $\left.d_{2}\right) \delta 177.1(C=0), 151.73\left(\mathrm{C}_{\mathrm{q}}\right), 145.83\left(\mathrm{C}_{\mathrm{q}}\right), 145.36$ $\left(\mathrm{C}_{\mathrm{q}}\right), 135.92\left(\mathrm{C}_{\mathrm{q}}\right), 132.94\left(\mathrm{C}_{\mathrm{q}}\right), 123.36(\mathrm{Ar}-\mathrm{CH}), 121.73(\mathrm{Ar}-\mathrm{CH}), 121.06(\mathrm{Ar}-\mathrm{CH}), 115.94(\mathrm{Ar}-$ $\mathrm{CH}), 115.88(\mathrm{Ar}-\mathrm{CH}), 45.62\left(\mathrm{br}, \mathrm{Ta}-\mathrm{N}\left(\mathrm{CH}_{3}\right)_{2}\right), 34.95\left(\mathrm{Ar}-\mathrm{CH}\left(\mathrm{CH}_{3}\right)_{2}\right), 31.60\left(\mathrm{Ar}-\mathrm{CH}\left(\mathrm{CH}_{3}\right)_{2}\right)$, $25.11\left(\mathrm{Ar}-\mathrm{CH}\left(\mathrm{CH}_{3}\right)_{2}\right), 24.15\left(\mathrm{Ar}-\mathrm{CH}\left(\mathrm{CH}_{3}\right)_{2}\right), 23.94\left(\mathrm{Ar}-\mathrm{CH}\left(\mathrm{CH}_{3}\right)_{2}\right)$.

FT-IR (ATR, cm-1) 3061, 2956, 2865, 2823, 2779, 1605, 1569, 1529, 1513, 1488, 1452, 1438, 1399, 1381, 1361, 1315, 1256, 1179, 1153, 1143, 1100, 1081, 1054, 1032, 968, 944, 895, 877, 861, 839, 802, 757, 741, 732, 718, 685, 652, 610, 569, 546, 516, 477, 453.

Melting Point: $286^{\circ} \mathrm{C}$ (decomposition).

Elemental Analysis Anal. Found: C, 62.07; H, 6.96; N, 7.56. Calc. ( $\left.\mathrm{C}_{48} \mathrm{H}_{66} \mathrm{~N}_{5} \mathrm{O}_{3} \mathrm{Ta}\right): \mathrm{C}, 62.26$; $\mathrm{H}$, 7.18; N, 7.56. 


\section{References}

(1) Ren, P.; Vechorkin, O.; Von Allmen, K.; Scopelliti, R.; Hu, X. A Structure-Activity Study of Ni-Catalyzed Alkyl-Axlkyl Kumada Coupling. Improved Catalysts for Coupling of Secondary Alkyl Halides. J. Am. Chem. Soc. 2011, 133 (18), 7084-7095.

(2) Dolomanov, O. V.; Bourhis, L. J.; Gildea, R. J.; Howard, J. A. K.; Puschmann, H. OLEX2: A Complete Structure Solution, Refinement and Analysis Program. J. Appl. Crystallogr. 2009, 42 (2), 339-341.

(3) Sheldrick, G. M. A Short History of SHELX. Acta Crystallogr. Sect. A Found. Crystallogr. 2008, 64 (1), 112-122.

(4) Sheldrick, G. M. SHELXT - Integrated Space-Group and Crystal-Structure Determination. Acta Crystallogr. Sect. A Found. Crystallogr. 2015, 71 (1), 3-8.

(5) Sheldrick, G. M. Crystal Structure Refinement with SHELXL. Acta Crystallogr. Sect. C Struct. Chem. 2015, 71 (Md), 3-8. 


\section{NMR Spectra - Ligands}

Figure S $2{ }^{1} \mathrm{H}$-NMR spectrum of $\mathbf{1 a}$ in $\mathrm{CDCl}_{3}$ at room temperature

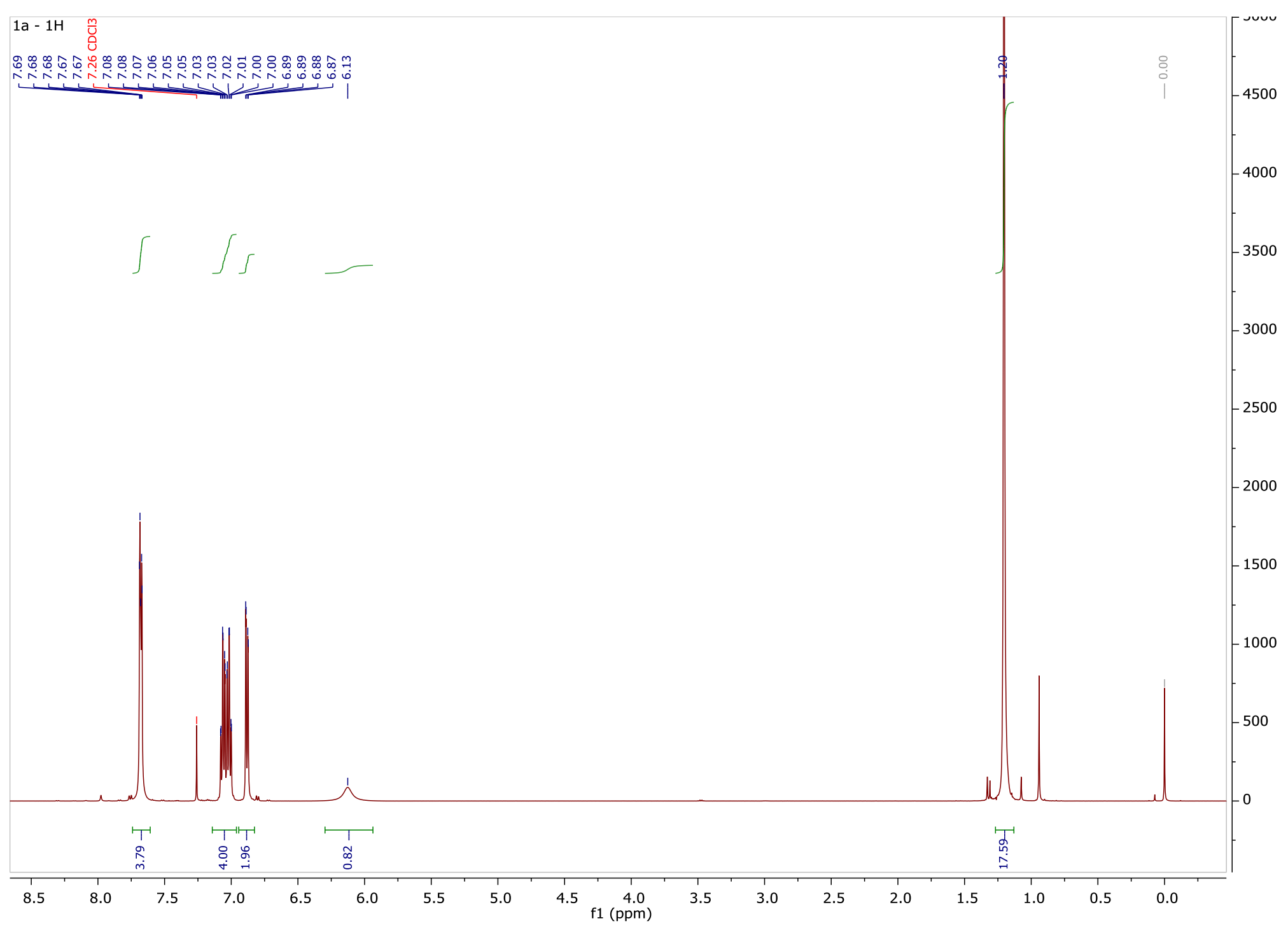


Figure S $3{ }^{13} \mathrm{C}$-NMR spectrum of $\mathbf{1 a}$ in $\mathrm{CDCl}_{3}$ at room temperature

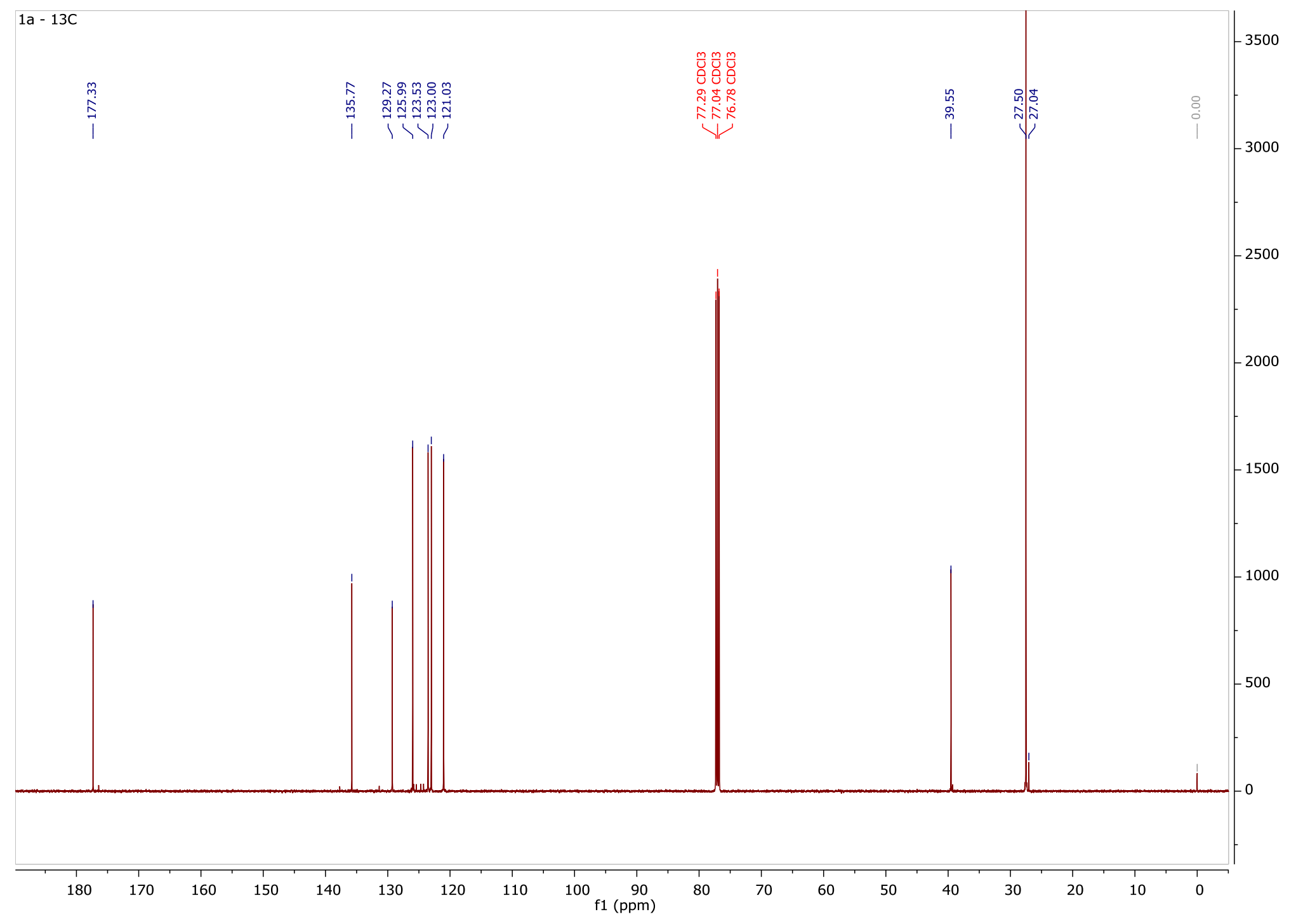


Figure $\mathbf{S} \mathbf{4} \mathrm{HSQC}$ spectrum of $\mathbf{1 a}$ in $\mathrm{CDCl}_{3}$ at room temperature

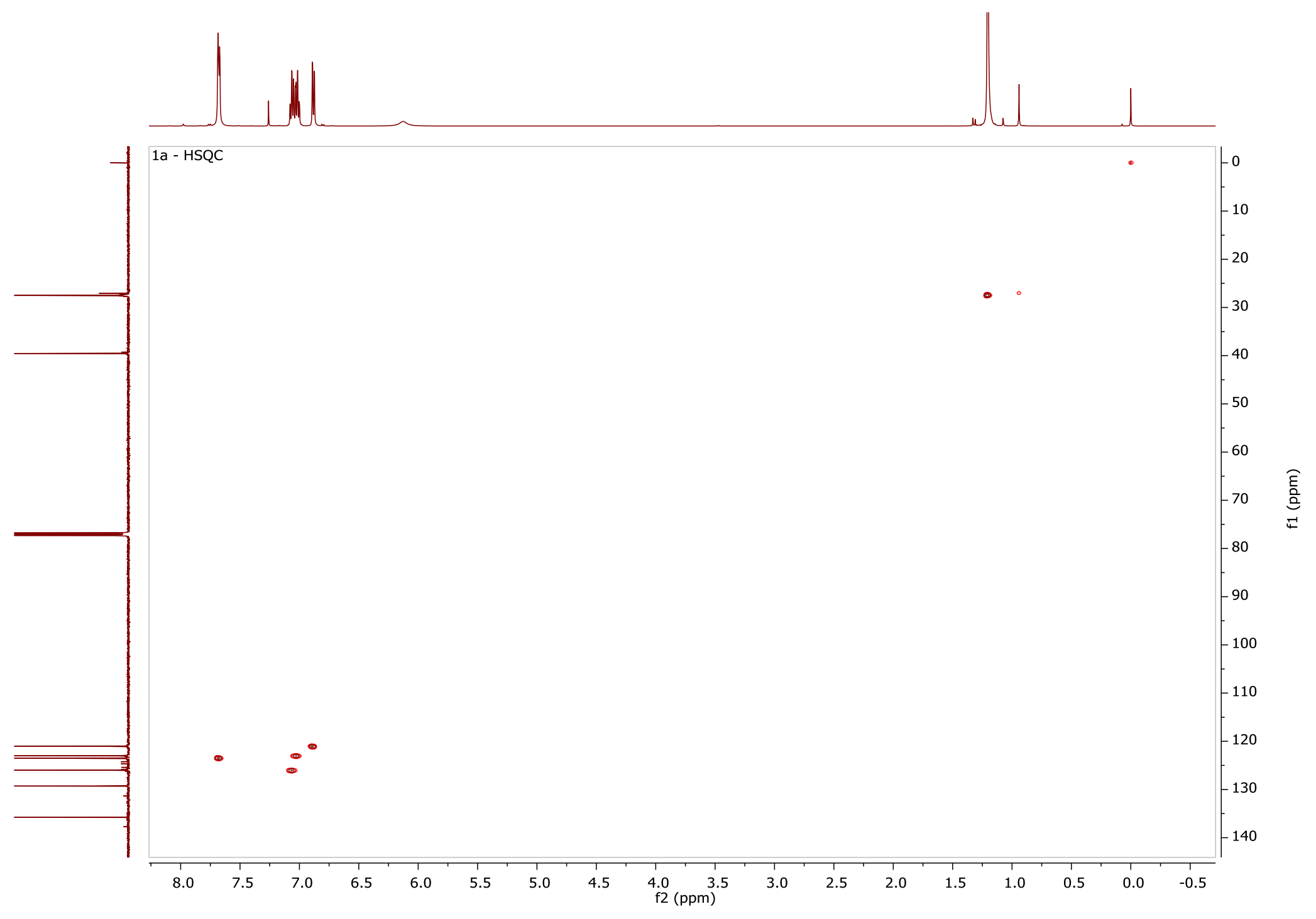


Figure $\mathbf{S} 5{ }^{1} \mathrm{H}-\mathrm{NMR}$ spectrum of $\mathbf{1} \mathbf{b}$ in $\mathrm{CDCl}_{3}$ at room temperature

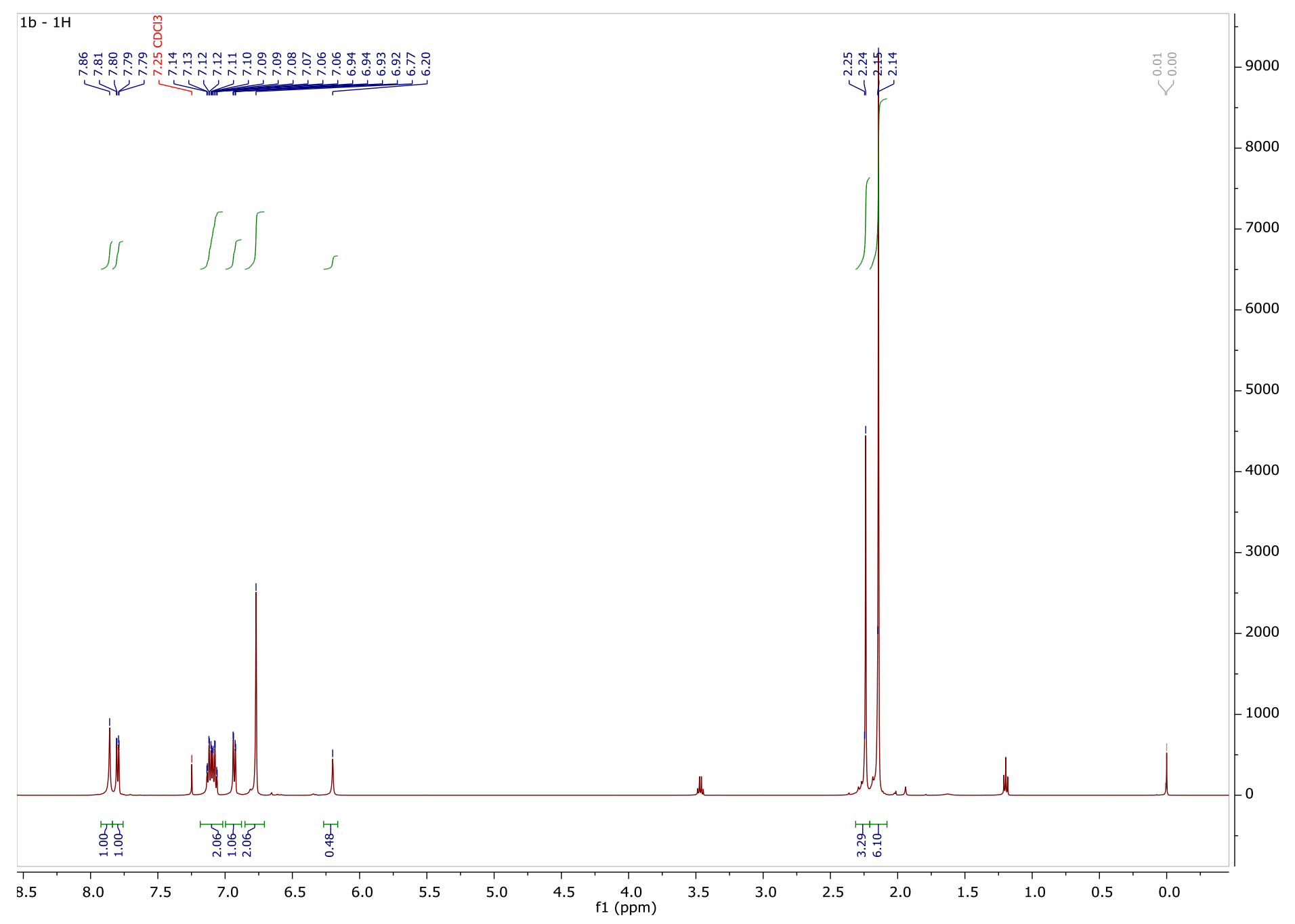


Figure $\mathbf{S} \mathbf{6}^{13} \mathrm{C}$-NMR spectrum of $\mathbf{1 b}$ in $\mathrm{CDCl}_{3}$ at room temperature

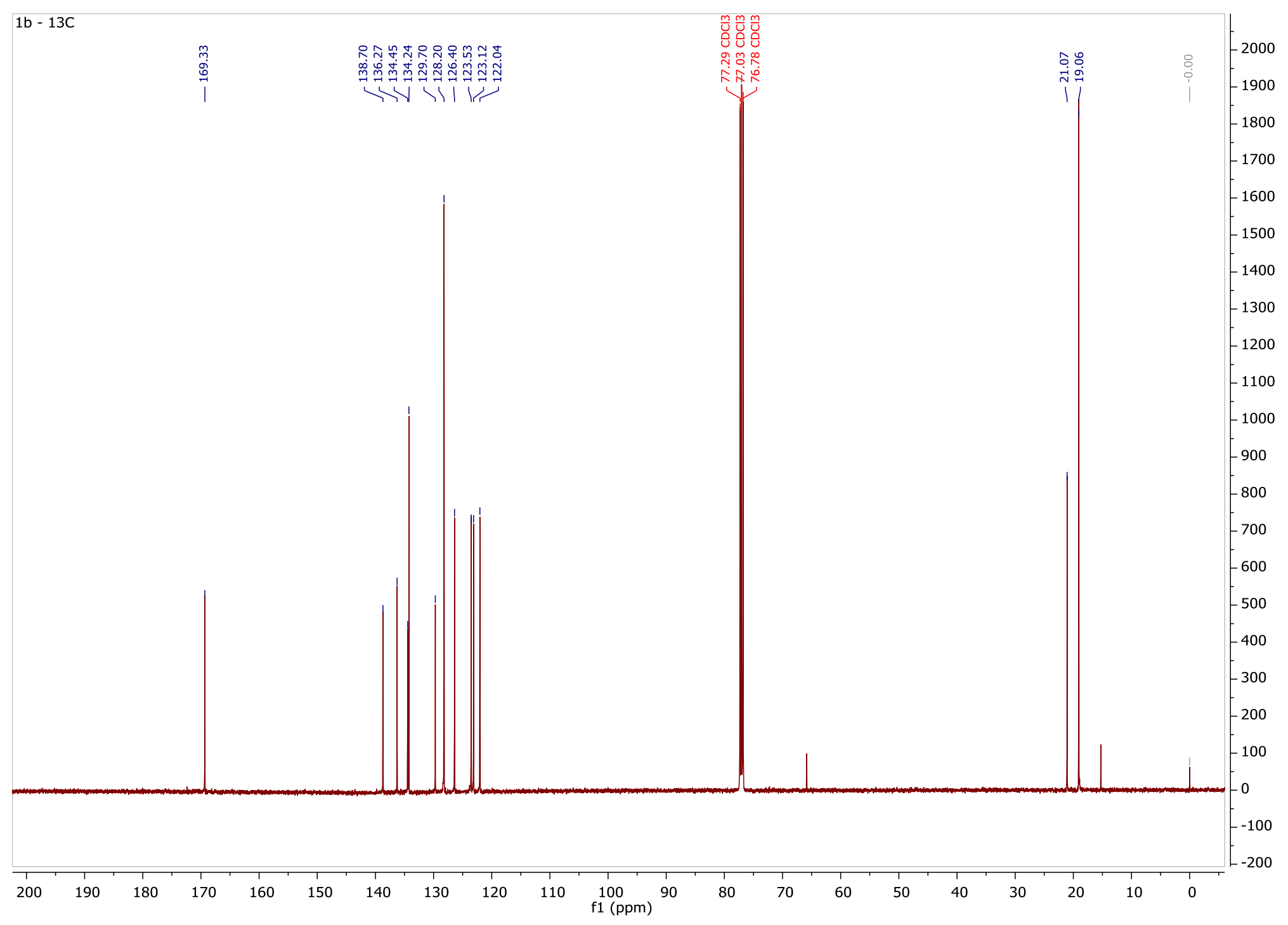


Figure $\mathbf{S} \mathbf{7} \mathrm{HSQC}-\mathrm{NMR}$ spectrum of $\mathbf{1 b}$ in $\mathrm{CDCl}_{3}$ at room temperature

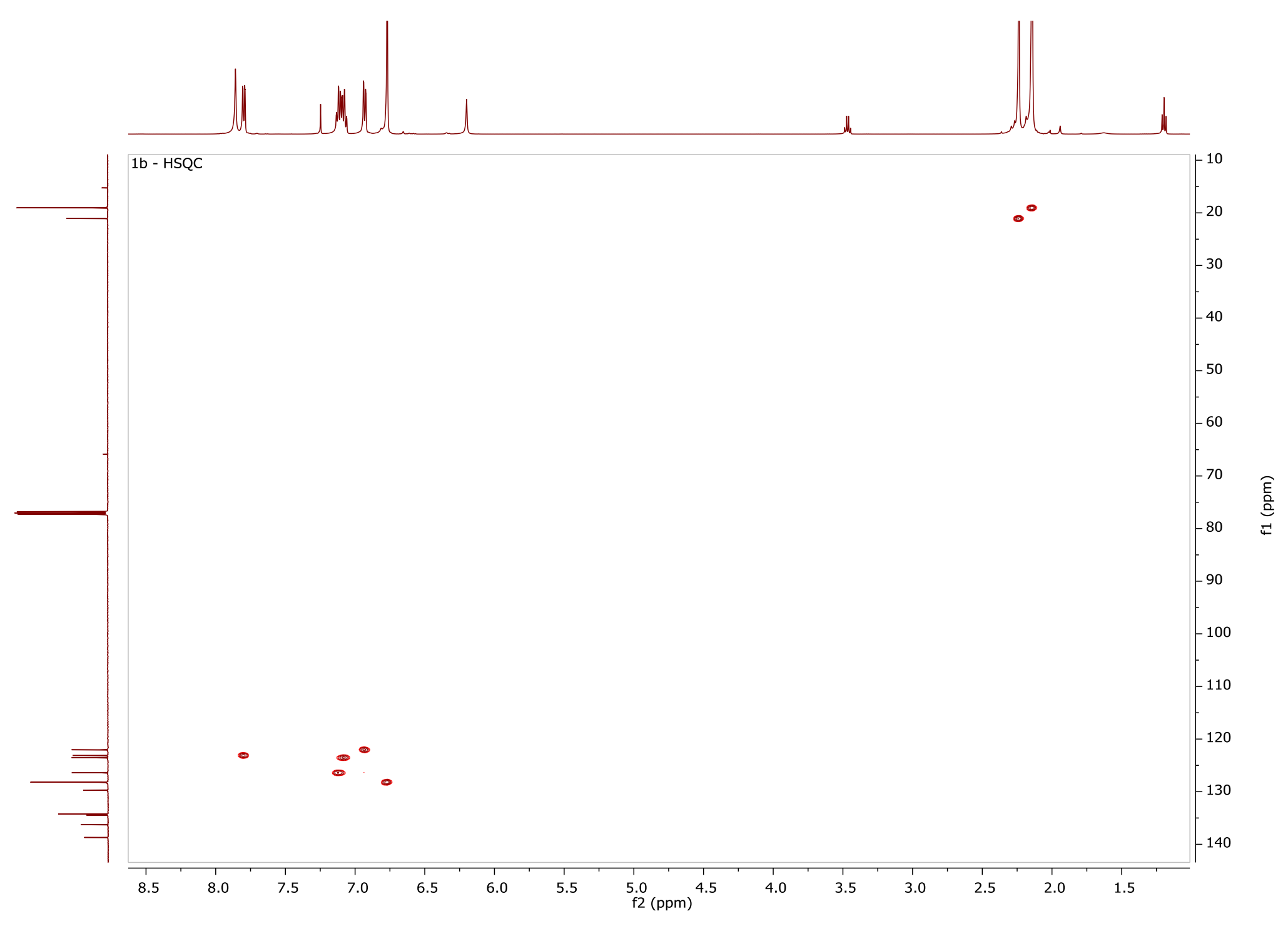


Figure $\mathrm{S} 8{ }^{1} \mathrm{H}$-NMR spectrum of $\mathbf{1 c}$ in $\mathrm{CDCl}_{3}$ at room temperature

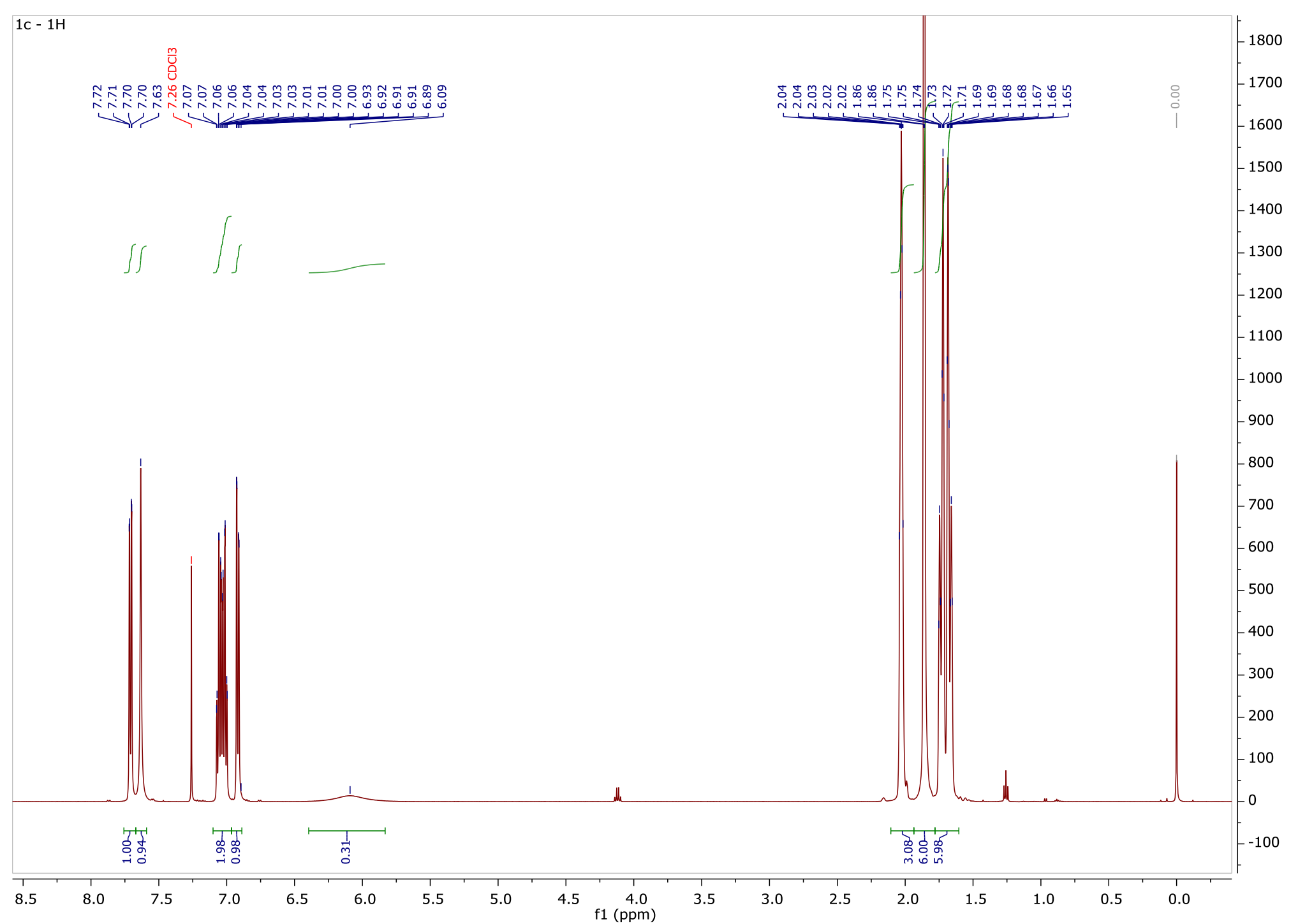


Figure S $9{ }^{13} \mathrm{C}$-NMR spectrum of $\mathbf{1 c}$ in $\mathrm{CDCl}_{3}$ at room temperature

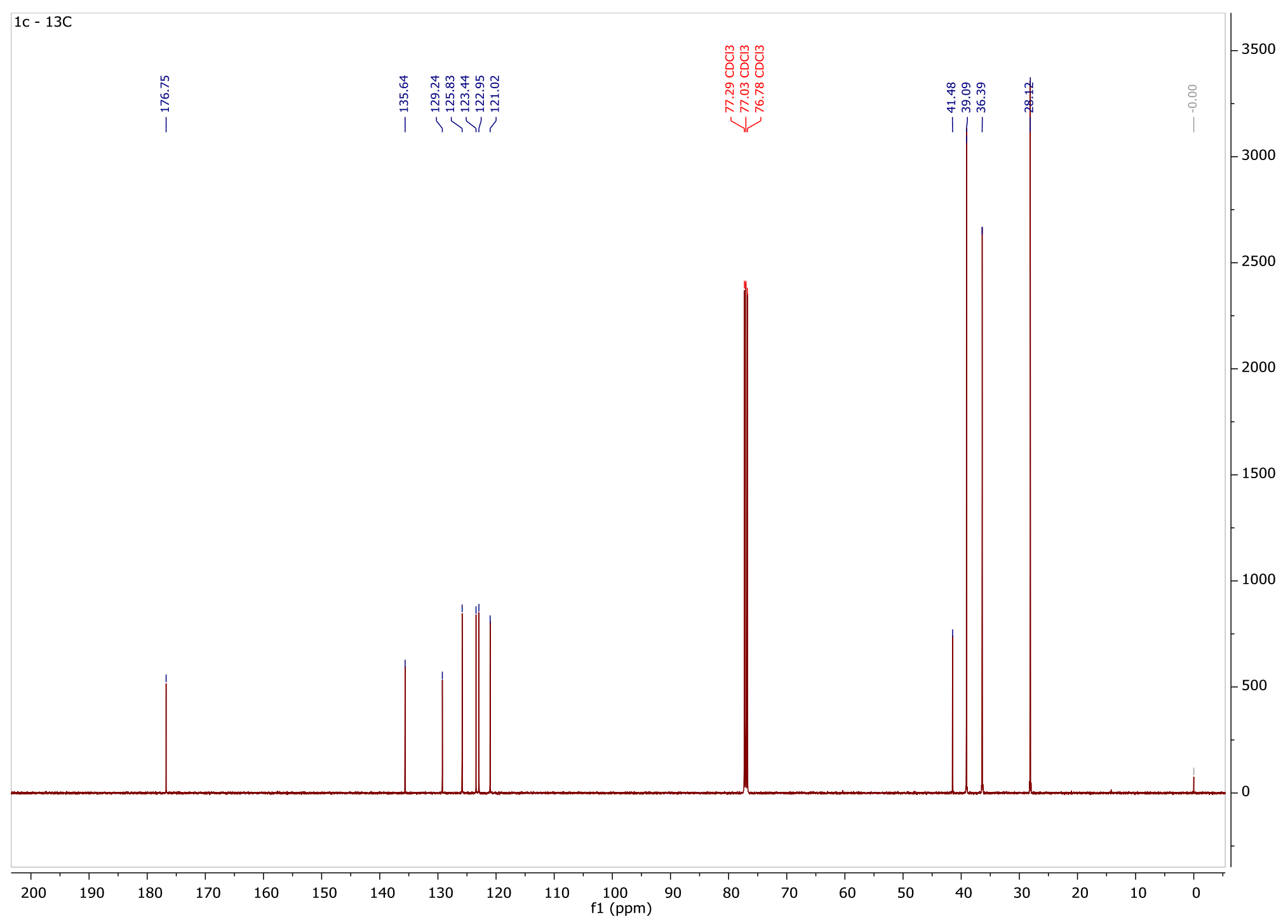


Figure $\mathbf{S} \mathbf{1 0} \mathrm{HSQC}-\mathrm{NMR}$ spectrum of $\mathbf{1 c}$ in $\mathrm{CDCl}_{3}$ at room temperature

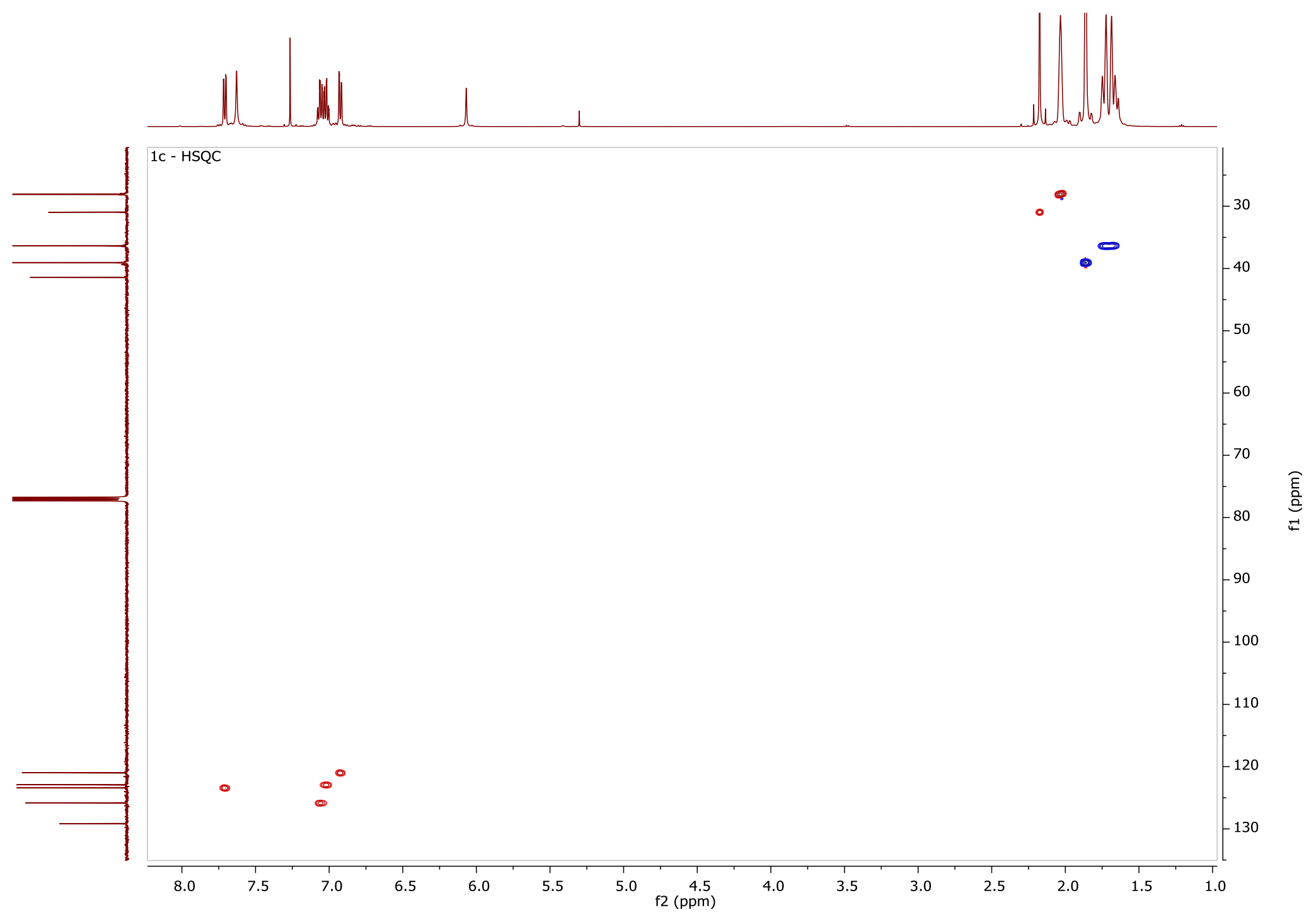


Figure S $11{ }^{1} \mathrm{H}-\mathrm{NMR}$ spectrum of $\mathbf{1 d}$ in $\mathrm{CDCl}_{3}$ at room temperature

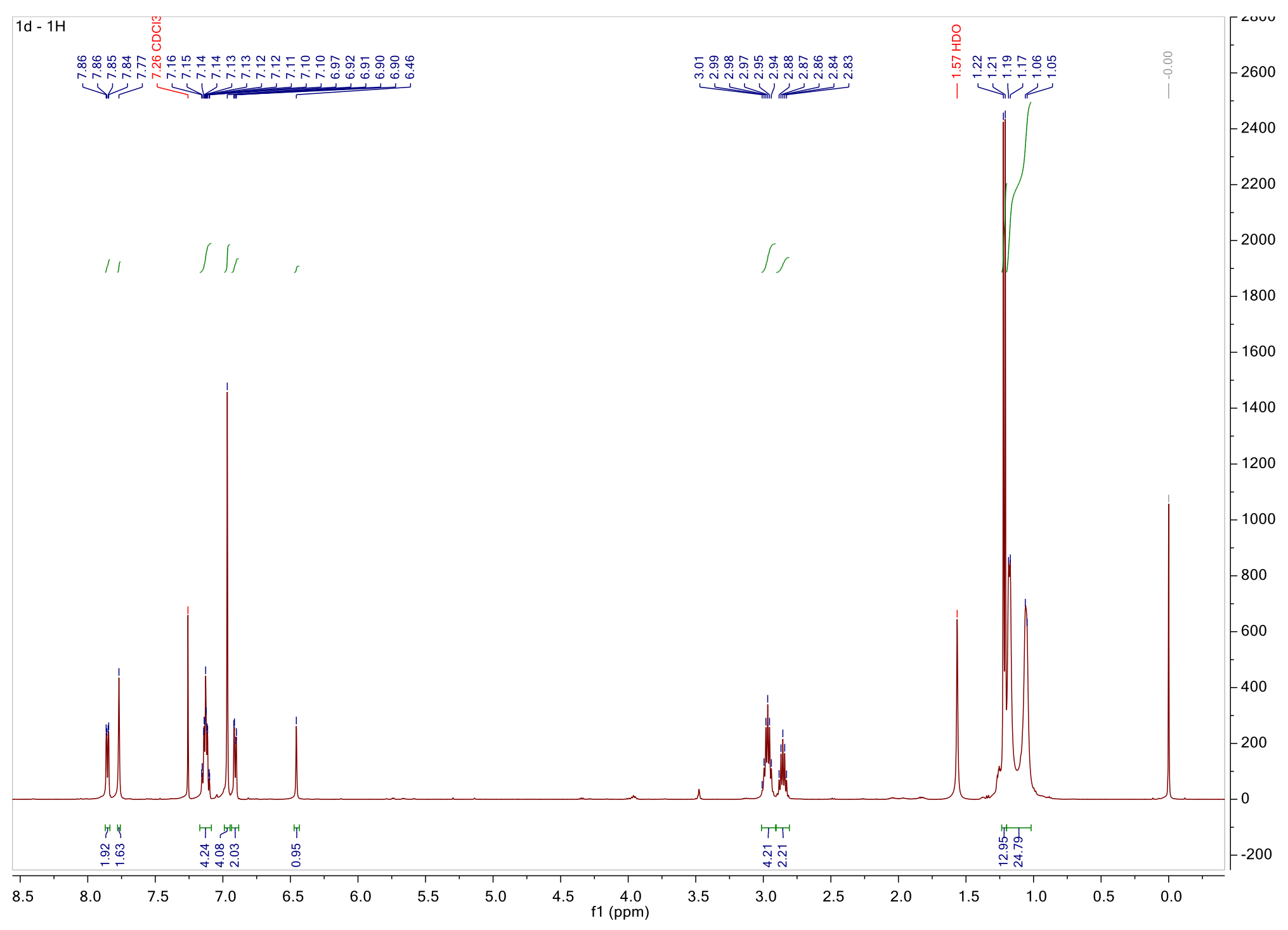


Figure $\mathbf{S} 12^{13} \mathrm{C}$-NMR spectrum of $\mathbf{1 d}$ in $\mathrm{CDCl}_{3}$ at room temperature

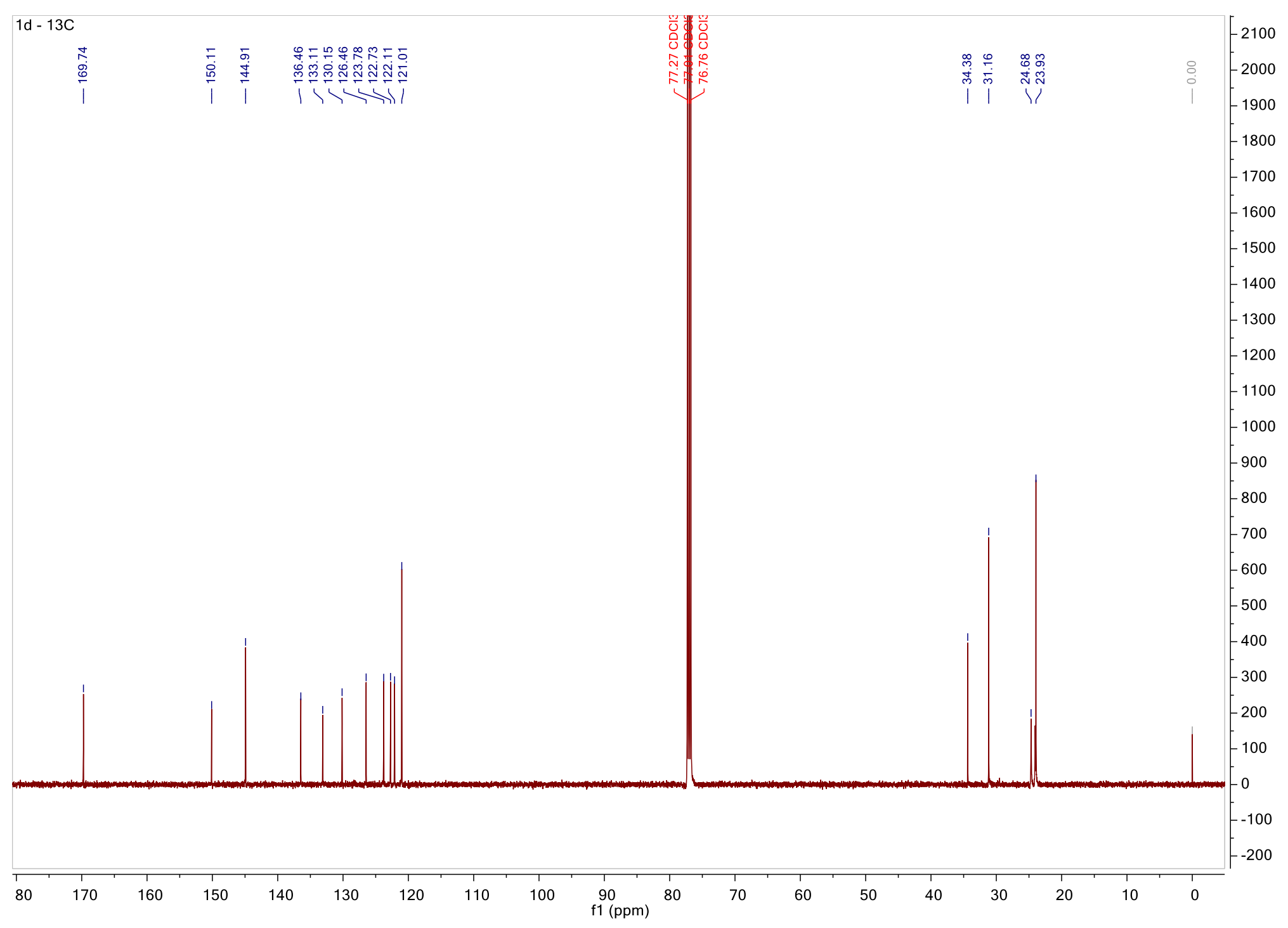


Figure S 13 HSQC-NMR spectrum of $\mathbf{1 d}$ in $\mathrm{CDCl}_{3}$ at room temperature

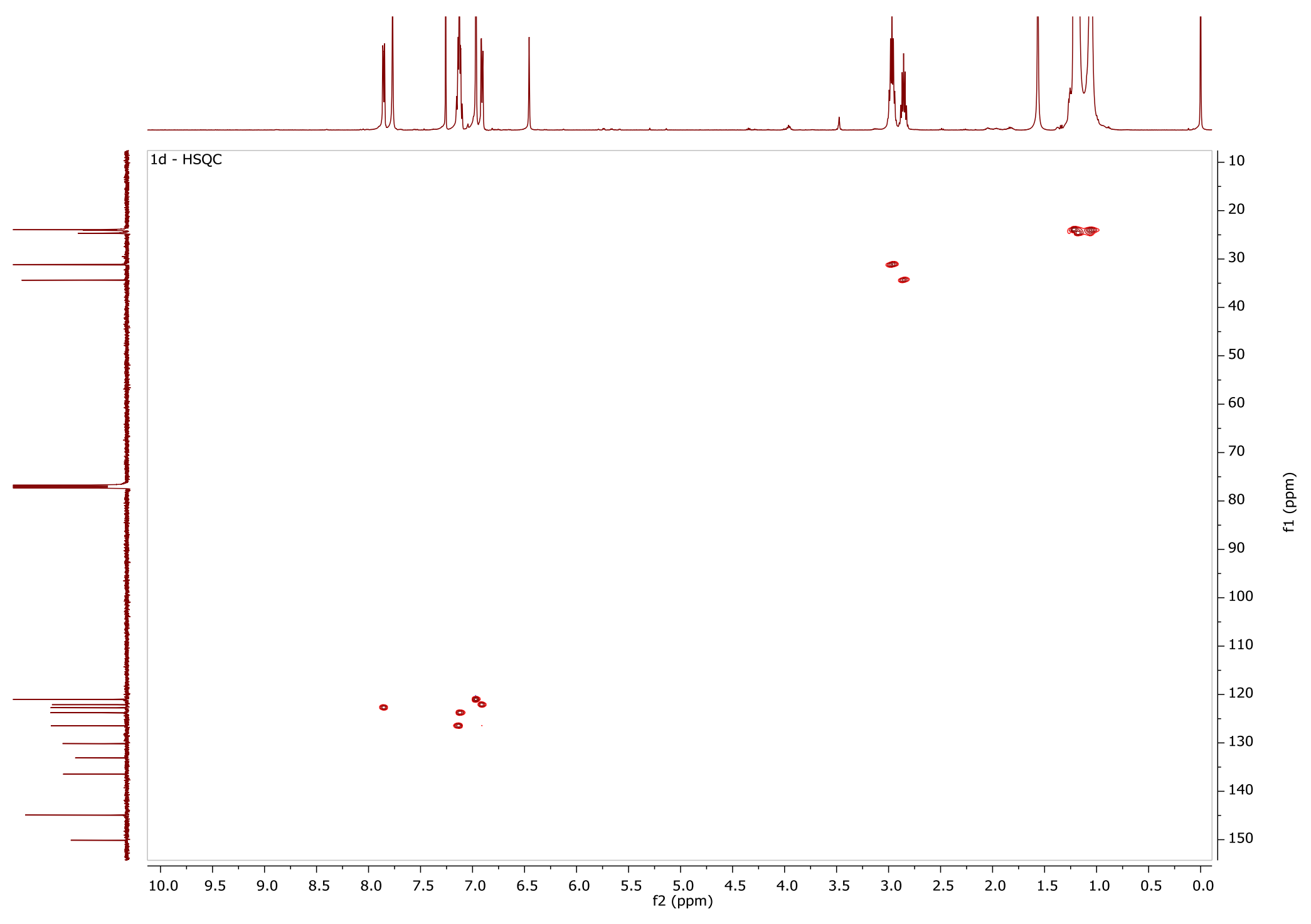


Figure S 14 COSY-NMR spectrum of $\mathbf{1 d}$ in $\mathrm{CDCl}_{3}$ at room temperature

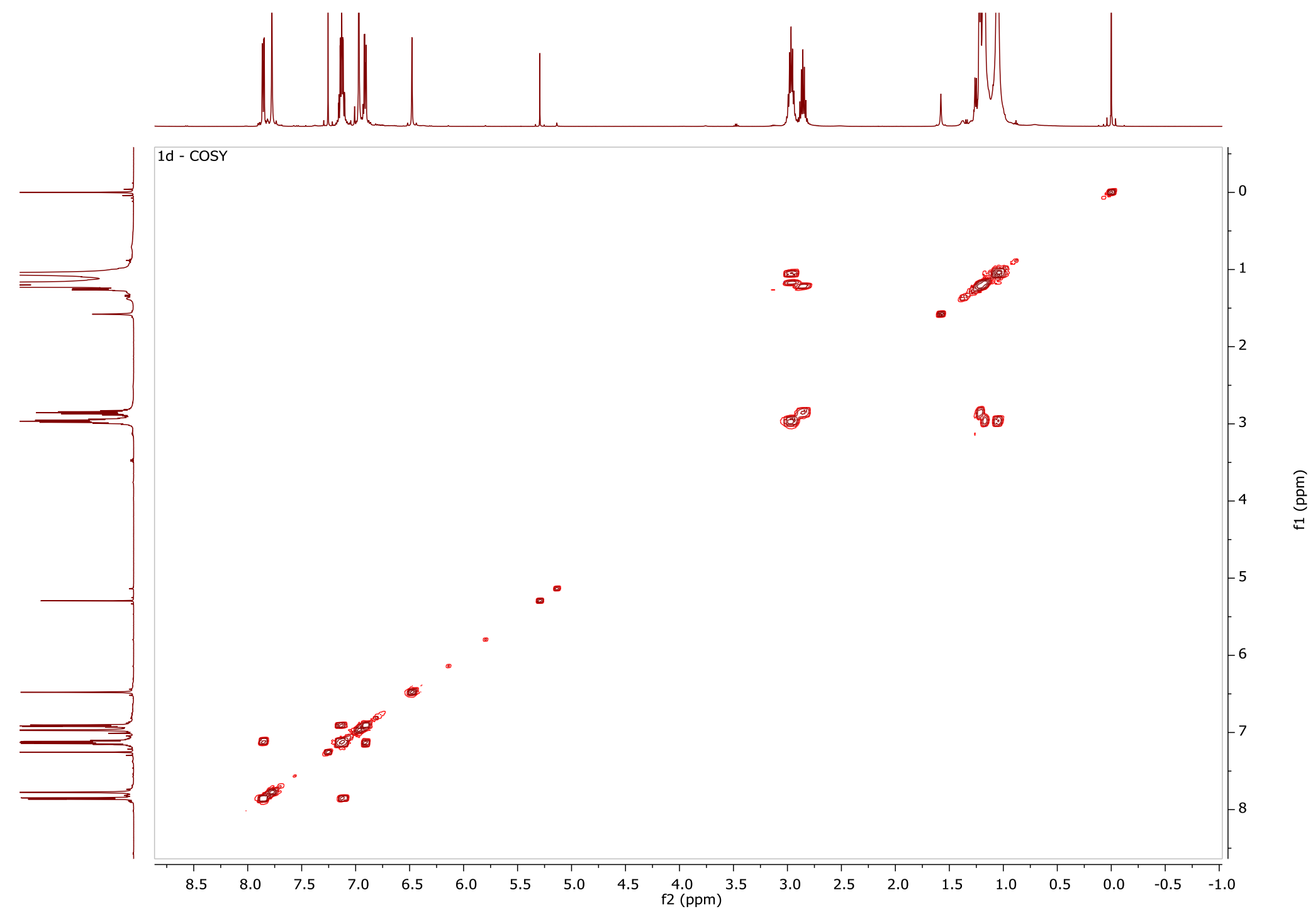


Figure $\mathbf{S} 15^{1} \mathrm{H}-\mathrm{NMR}$ spectrum of $\mathbf{1 e}$ in $\mathrm{CDCl}_{3}$ at room temperature

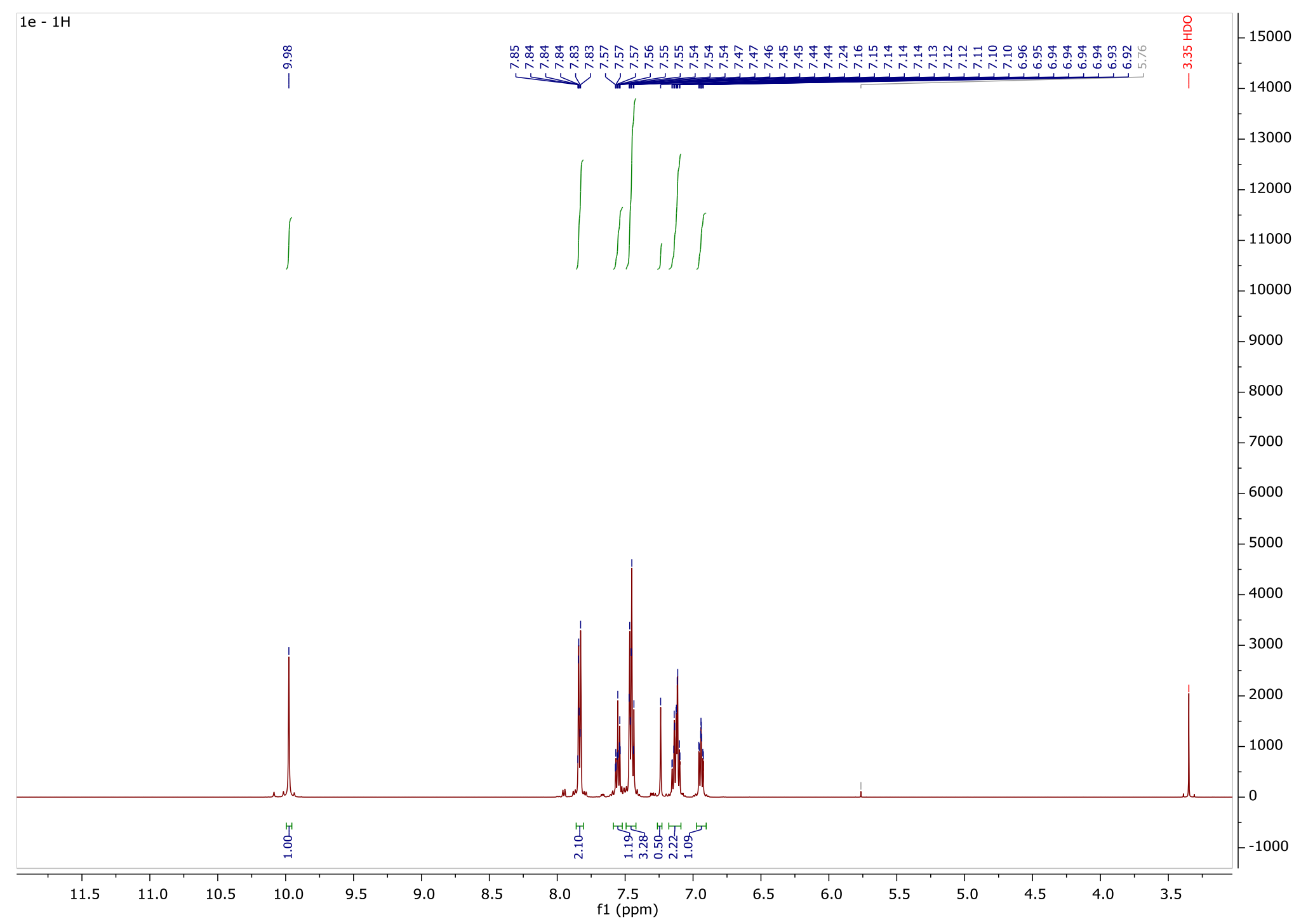


Figure $\mathbf{S} 16^{13} \mathrm{C}$-NMR spectrum of $1 \mathrm{e}$ in $\mathrm{CDCl}_{3}$ at room temperature

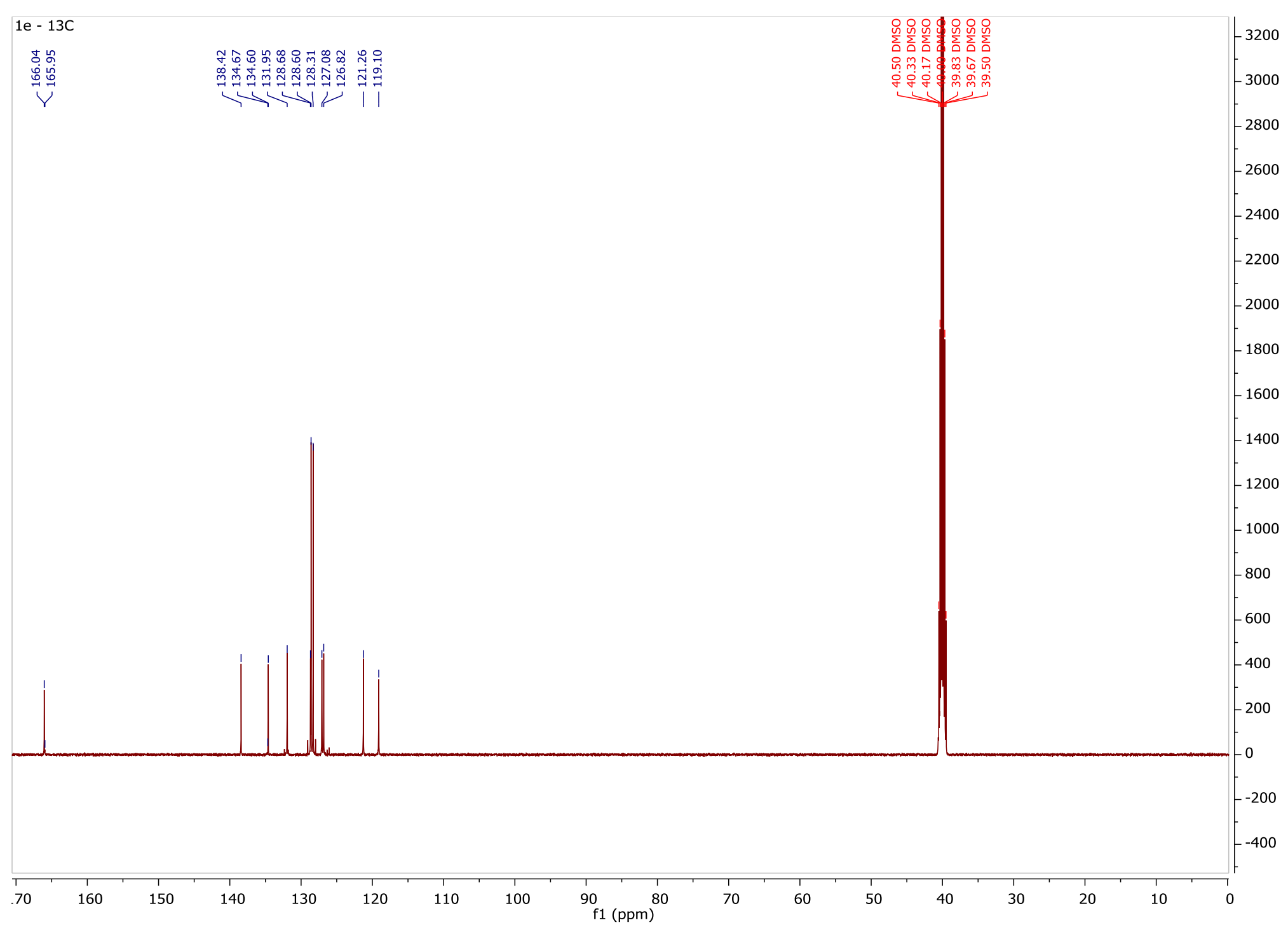


Figure S 17 HSQC-NMR spectrum of $\mathbf{1 e}$ in $\mathrm{CDCl}_{3}$ at room temperature

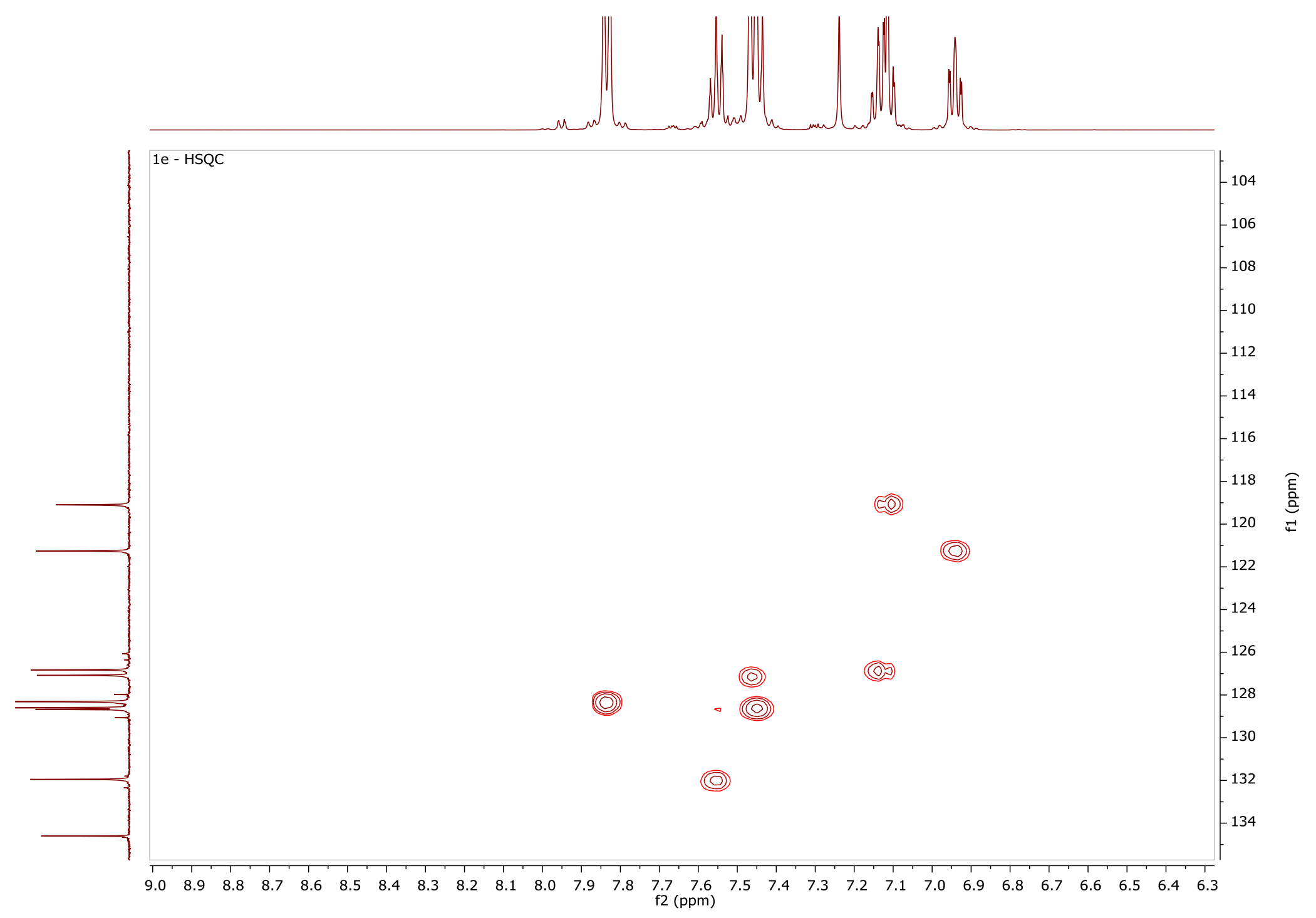


Figure $\mathbf{S} 18{ }^{1} \mathrm{H}-\mathrm{NMR}$ spectrum of 8 in $\mathrm{C}_{6} \mathrm{D}_{6}$ at room temperature

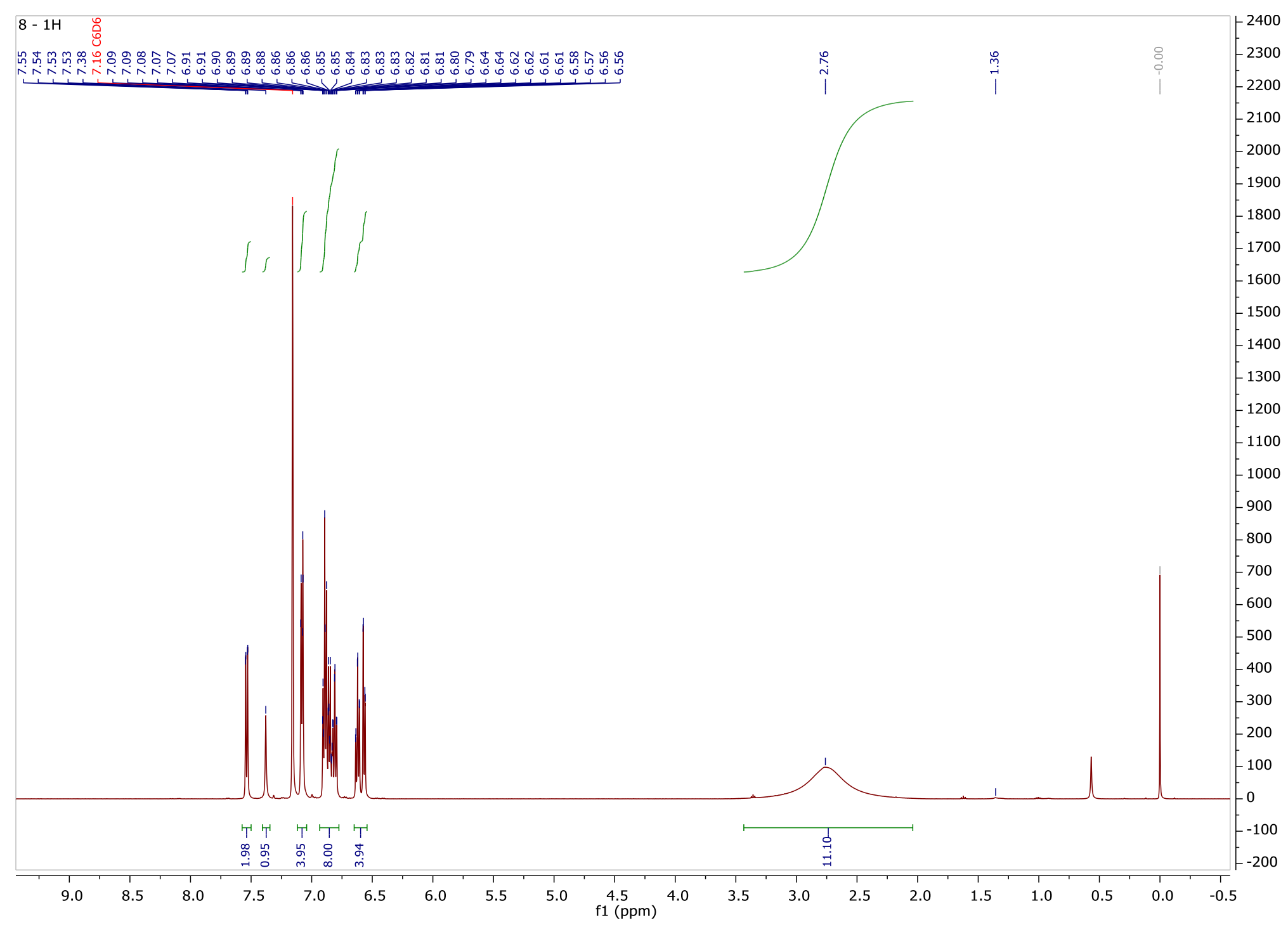


Figure $\mathbf{S} 19^{13} \mathrm{C}-\mathrm{NMR}$ spectrum of 8 in $\mathrm{C}_{6} \mathrm{D}_{6}$ at room temperature

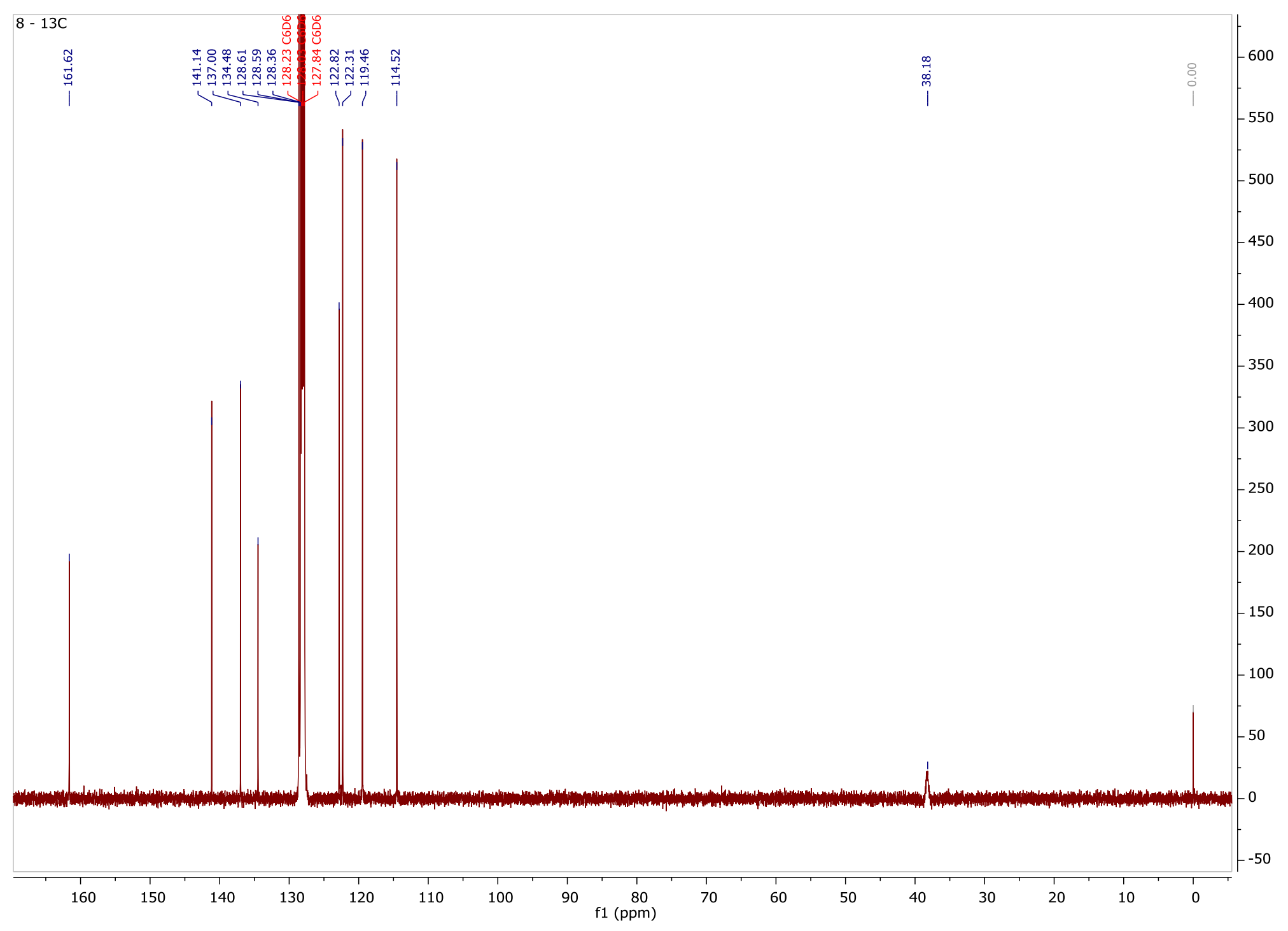


Figure S 20 HSQC-NMR spectrum of 8 in $\mathrm{C}_{6} \mathrm{D}_{6}$ at room temperature

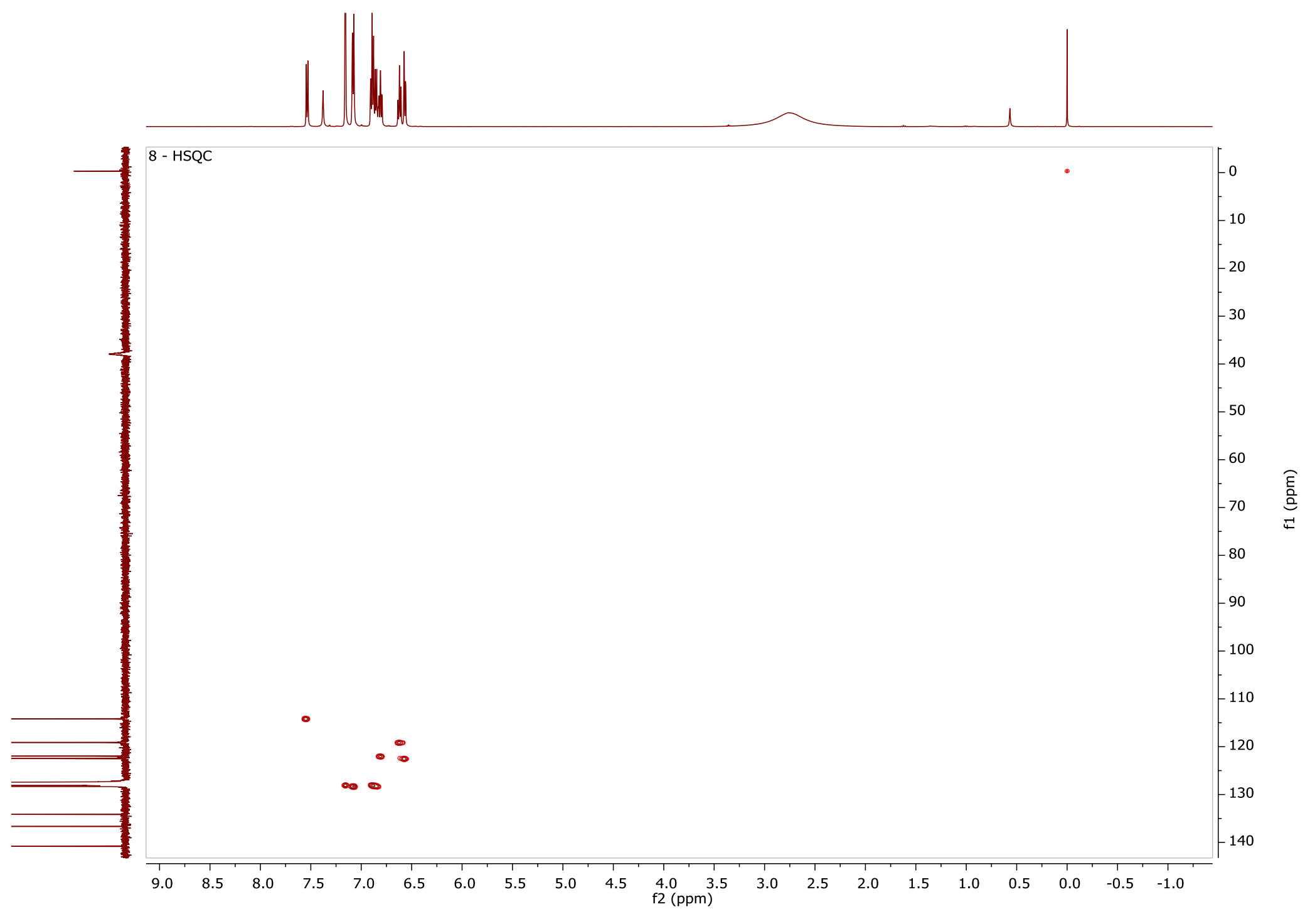


NMR Spectra - Metal Complexes 
Figure S $21{ }^{1} \mathrm{H}-\mathrm{NMR}$ spectrum of $\mathbf{2 a}$ in $\mathrm{C}_{6} \mathrm{D}_{6}$ at room temperature.

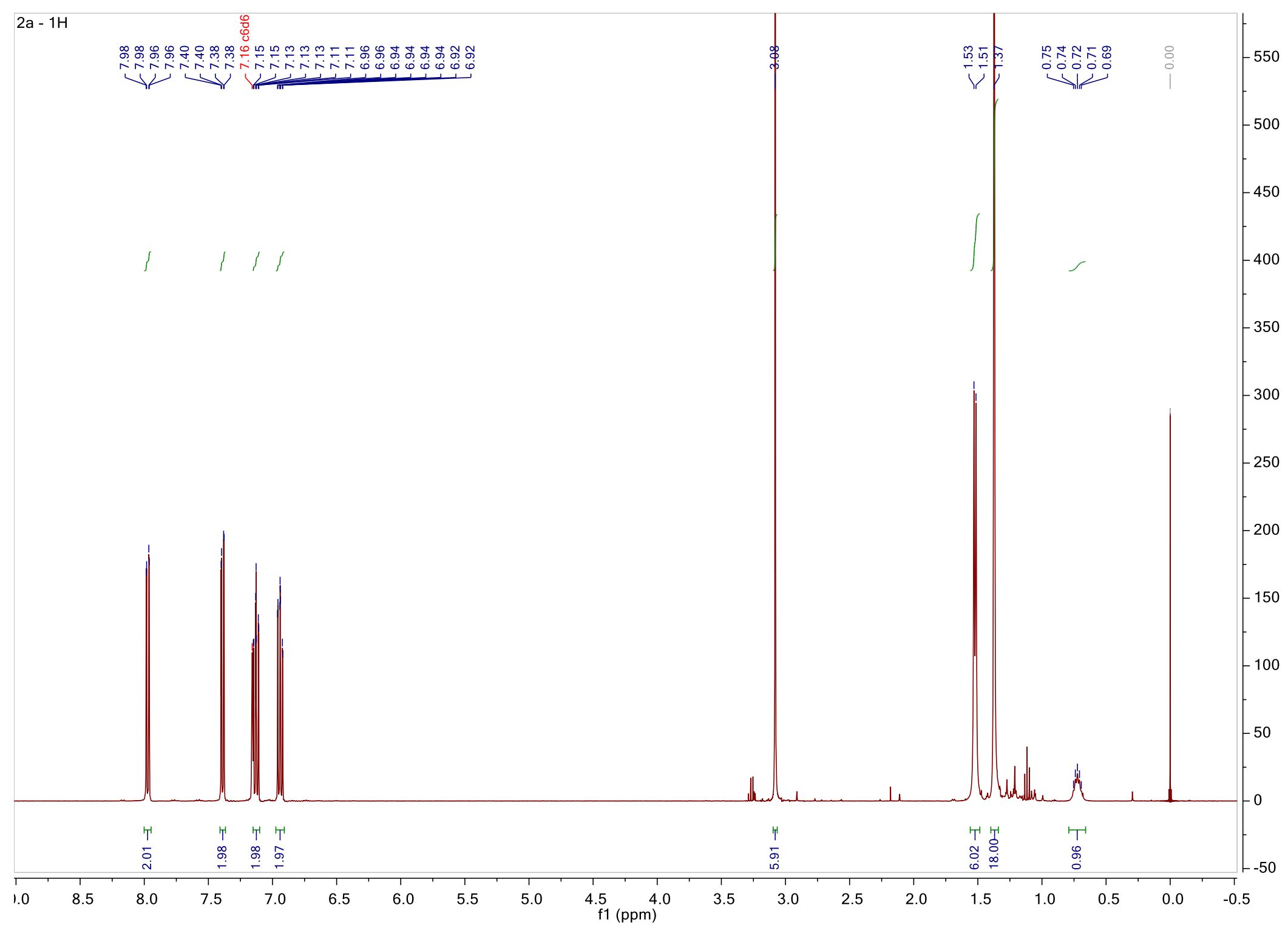


Figure $\mathbf{S} 22{ }^{13} \mathrm{C}-\mathrm{NMR}$ spectrum of $\mathbf{2 a}$ in $\mathrm{C}_{6} \mathrm{D}_{6}$ at room temperature.

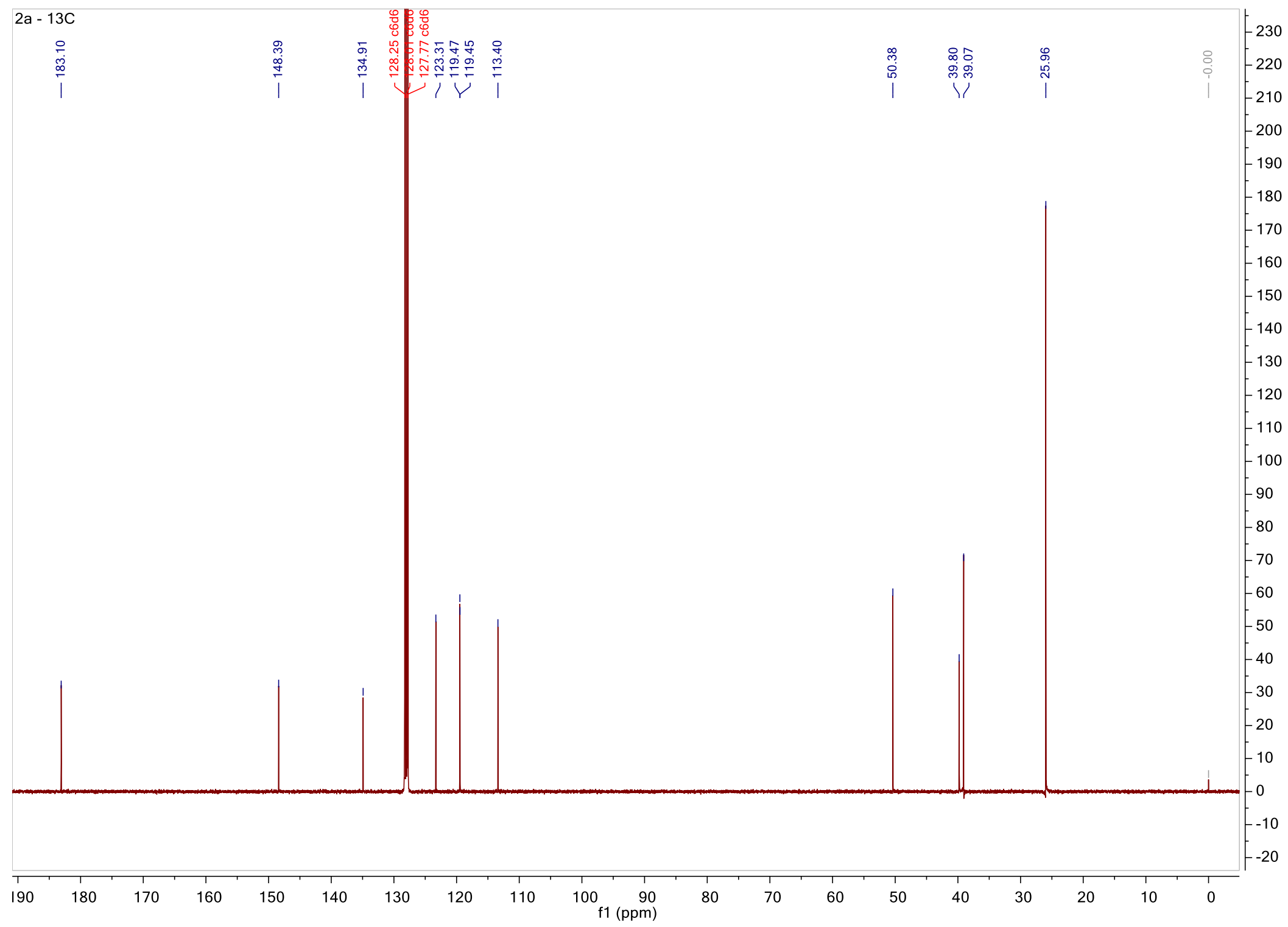


Figure $\mathbf{S} 23 \mathrm{HSQC}-\mathrm{NMR}$ spectrum of $\mathbf{2 a}$ in $\mathrm{C}_{6} \mathrm{D}_{6}$ at room temperature.

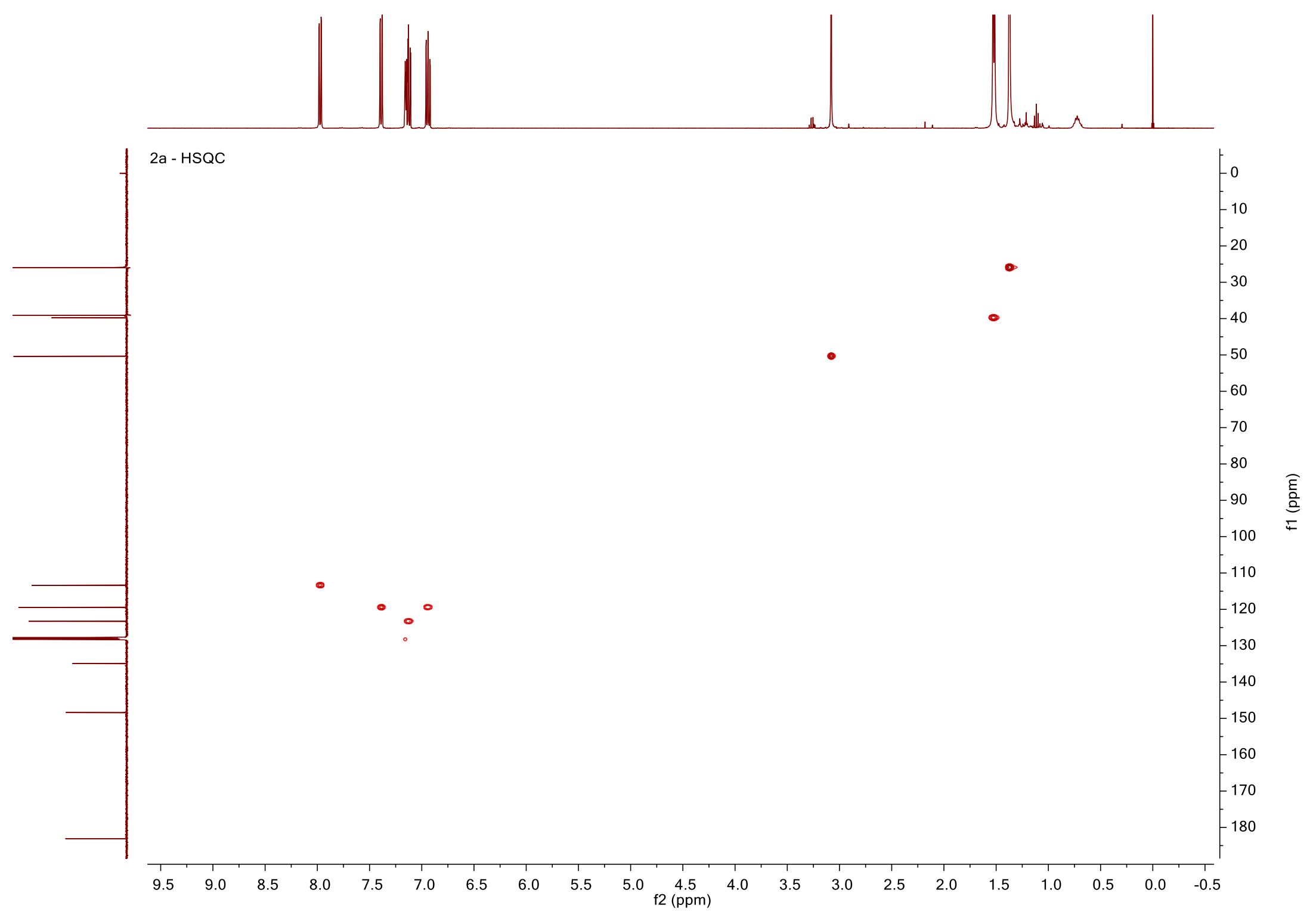


Figure $\mathbf{S} 24^{1} \mathrm{H}-\mathrm{NMR}$ spectrum of $2 \mathrm{a}$ in $\mathrm{C}_{6} \mathrm{D}_{6}$ at $70^{\circ} \mathrm{C}$.

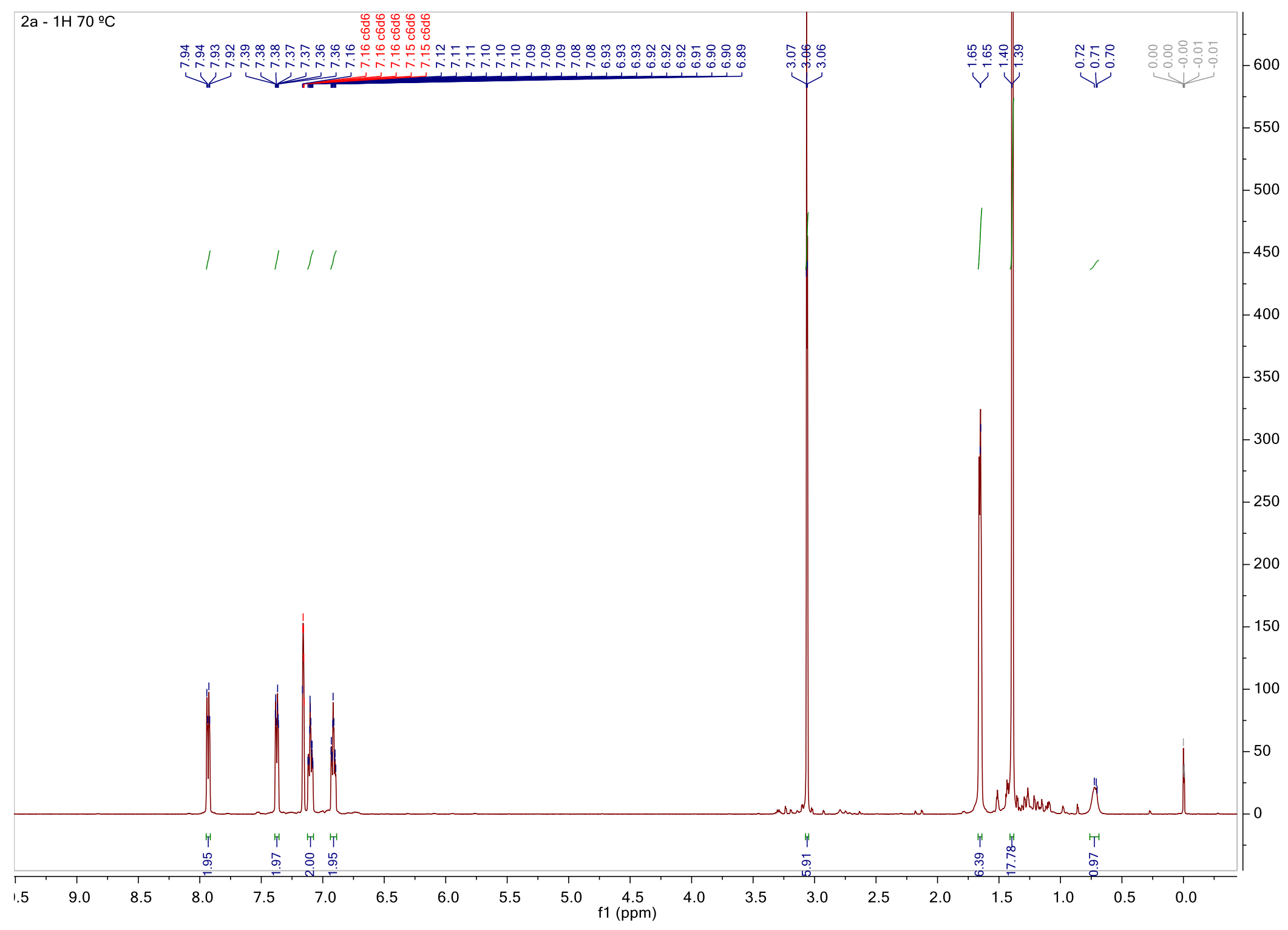


Figure $\mathbf{S} 25{ }^{1} \mathrm{H}-\mathrm{NMR}$ spectrum of $\mathbf{2 b}$ in $\mathrm{C}_{6} \mathrm{D}_{6}$ at room temperature.

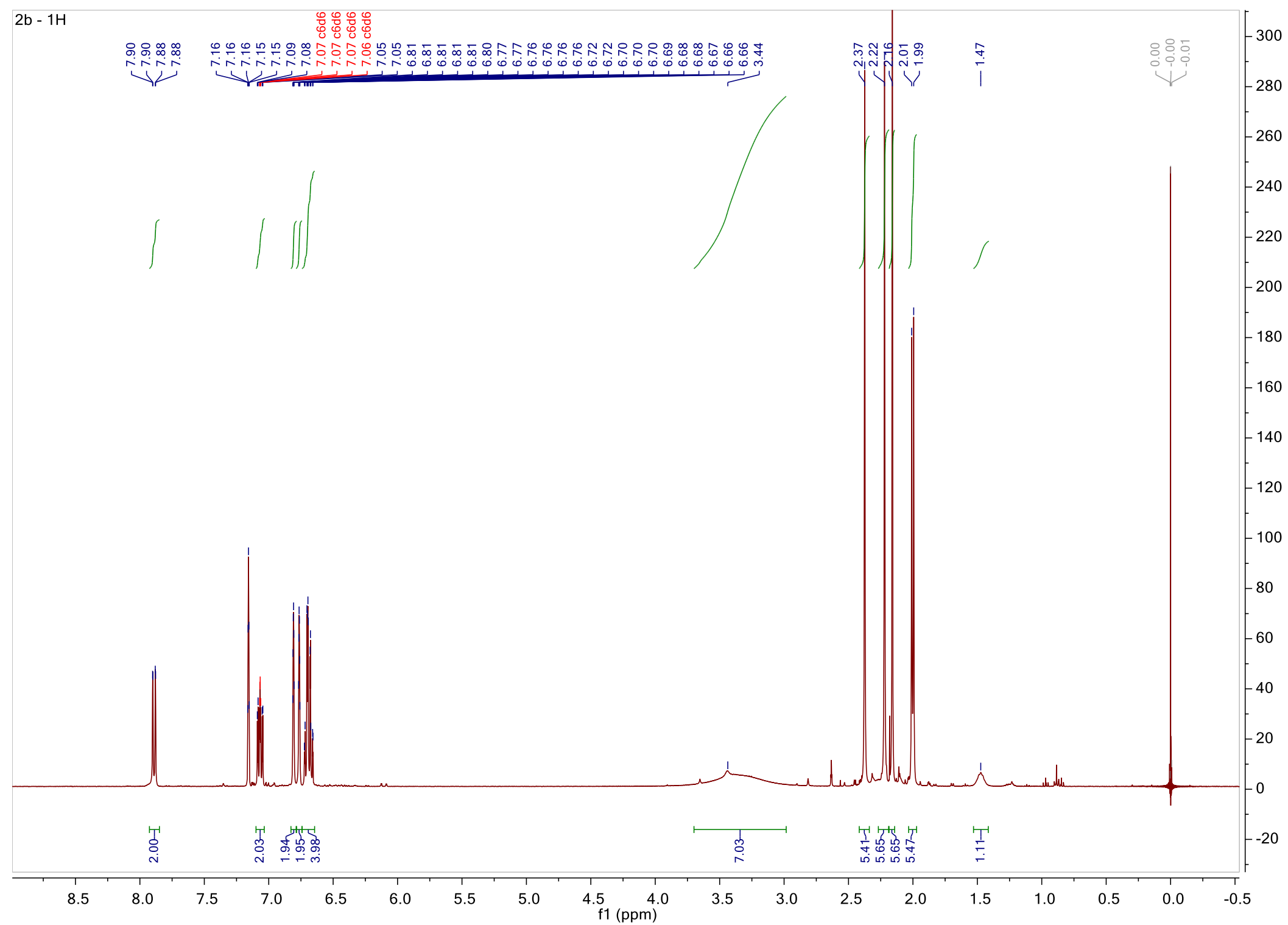


Figure $\mathbf{S} 2 \mathbf{6}^{13} \mathrm{C}$-NMR spectrum of $\mathbf{2 b}$ in $\mathrm{C}_{6} \mathrm{D}_{6}$ at room temperature.

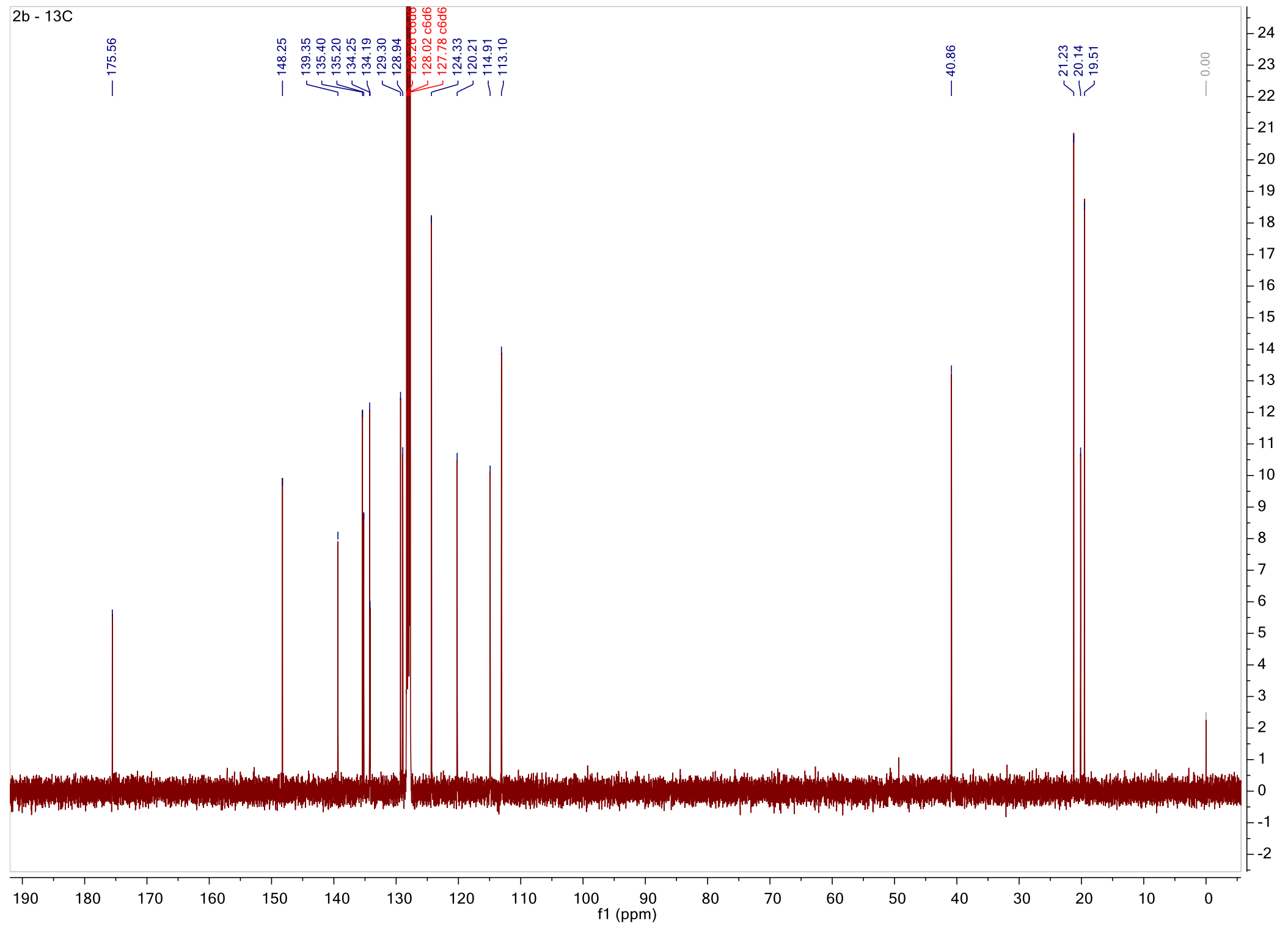


Figure S 27 HSQC-NMR spectrum of $\mathbf{2} \mathbf{b}$ in $\mathrm{C}_{6} \mathrm{D}_{6}$ at room temperature.

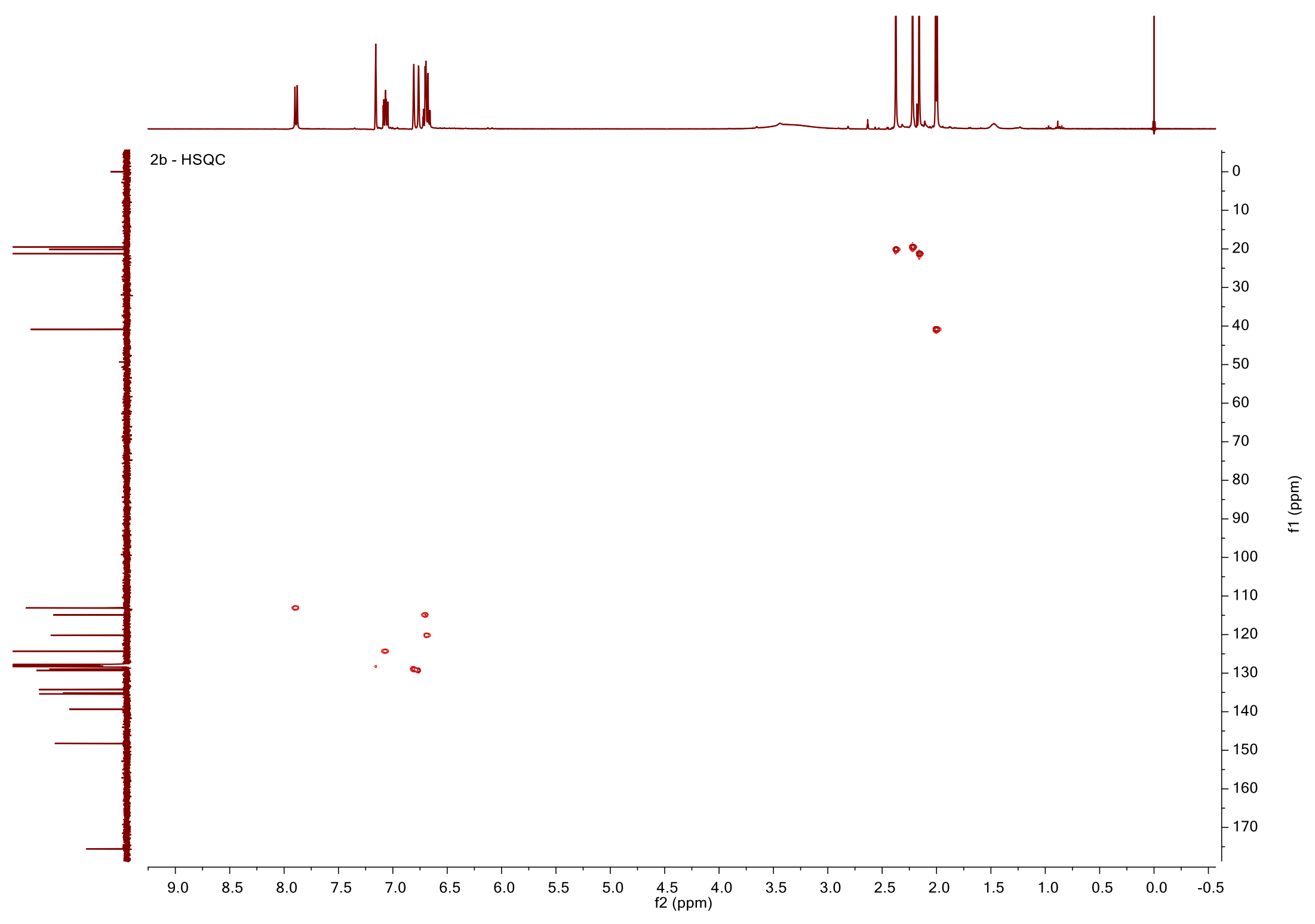


Figure $\mathbf{S} 28^{1} \mathrm{H}-\mathrm{NMR}$ spectrum of $\mathbf{2 b}$ in $\mathrm{C}_{6} \mathrm{D}_{6}$ at $70^{\circ} \mathrm{C}$.

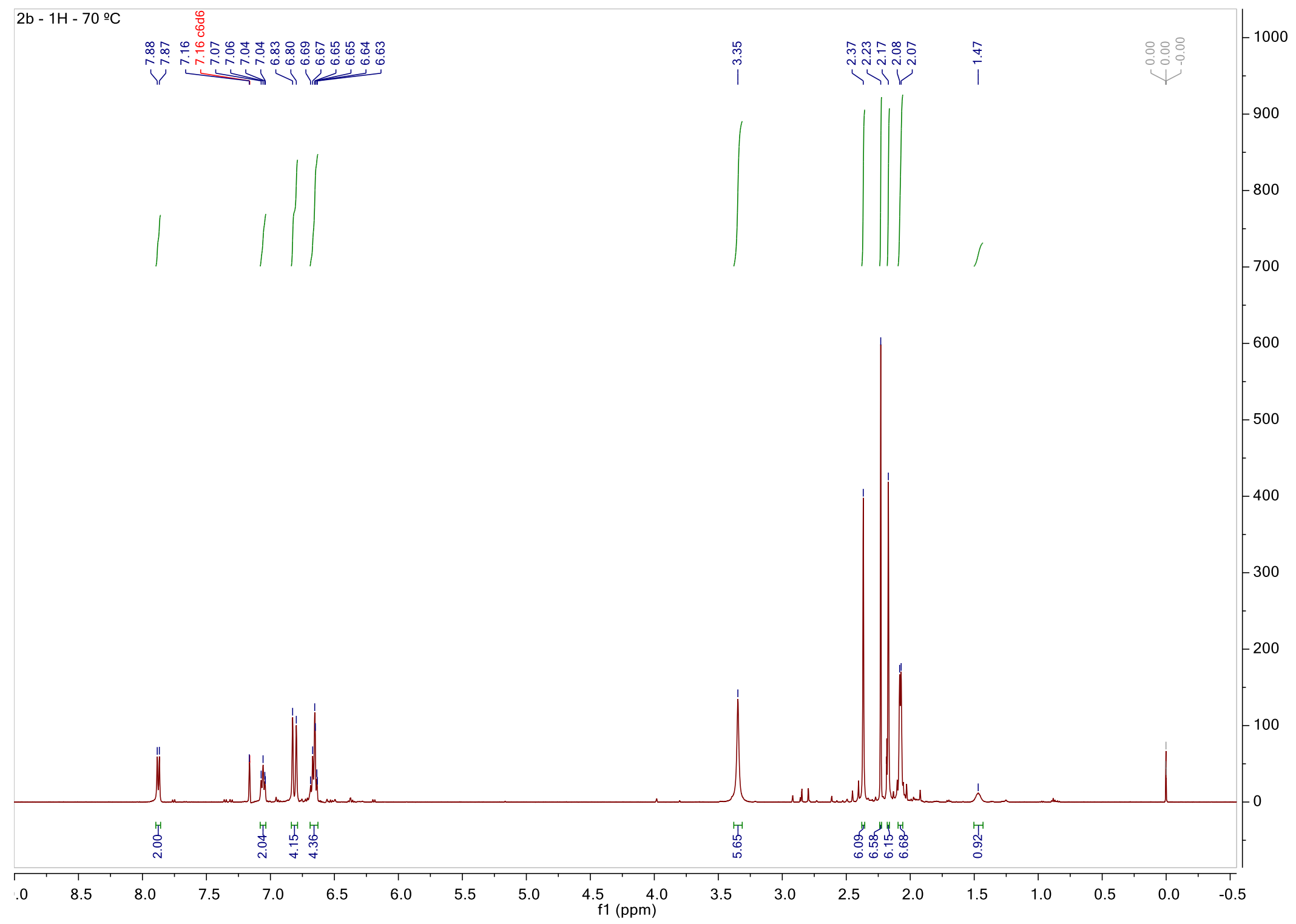


Figure $\mathbf{S} 29{ }^{1} \mathrm{H}-\mathrm{NMR}$ spectrum of $2 \mathbf{c}$ in $\mathrm{C}_{6} \mathrm{D}_{6}$ at room temperature.

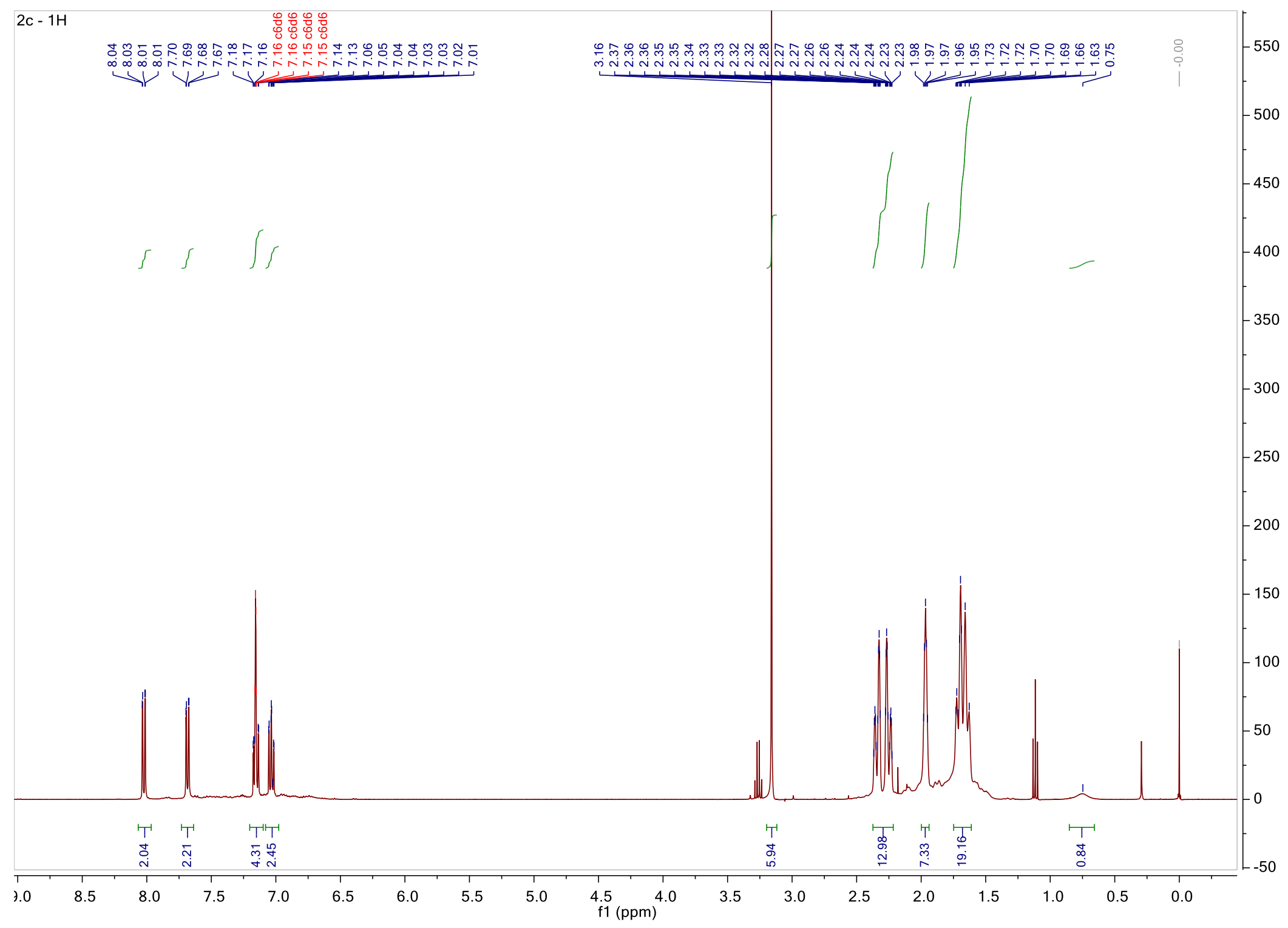


Figure $\mathbf{S} 30^{13} \mathrm{C}-\mathrm{NMR}$ spectrum of $2 \mathbf{c}$ in $\mathrm{C}_{6} \mathrm{D}_{6}$ at room temperature.

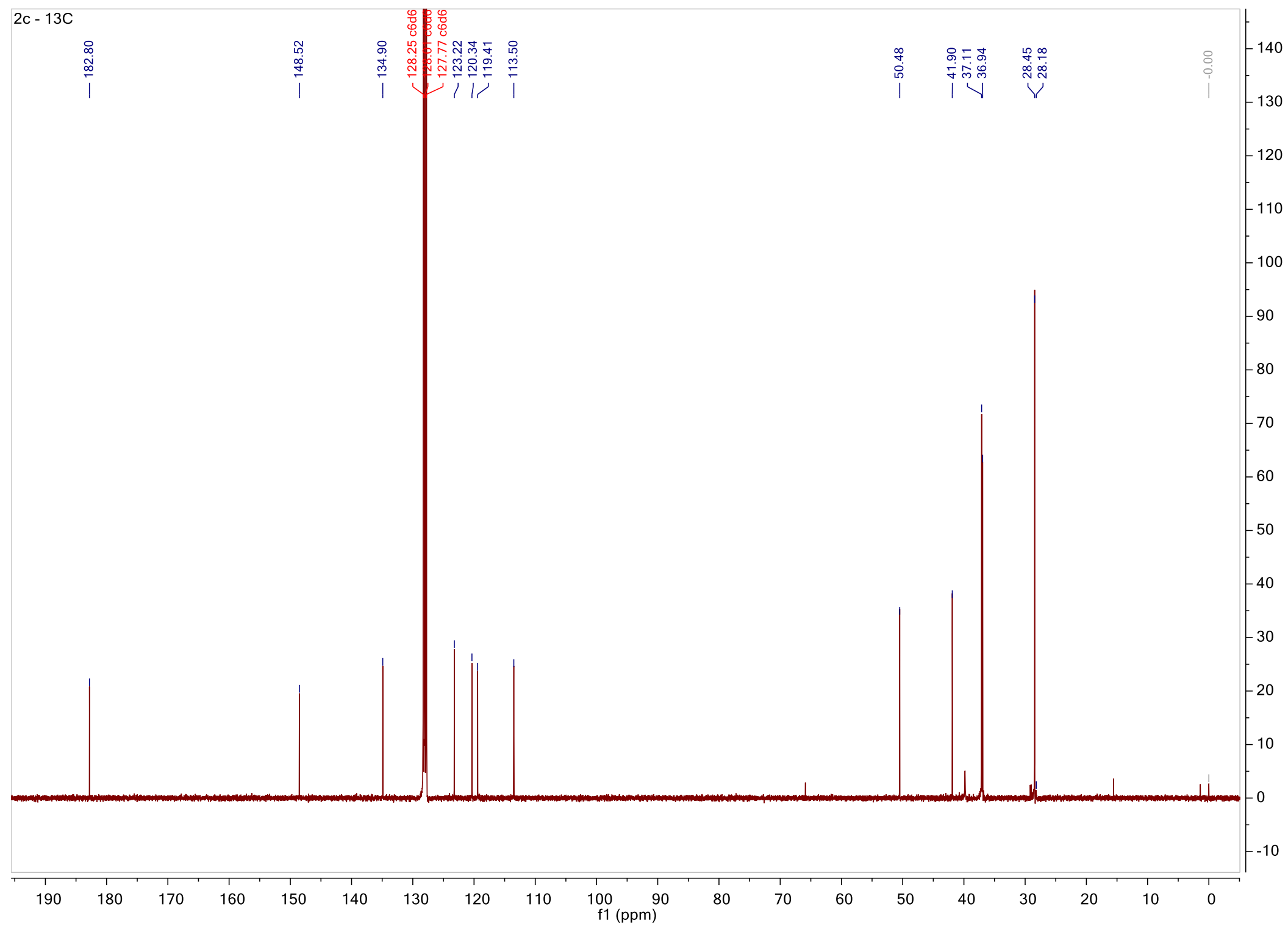


Figure S 31 HSQC-NMR spectrum of $2 \mathbf{c}$ in $\mathrm{C}_{6} \mathrm{D}_{6}$ at room temperature.

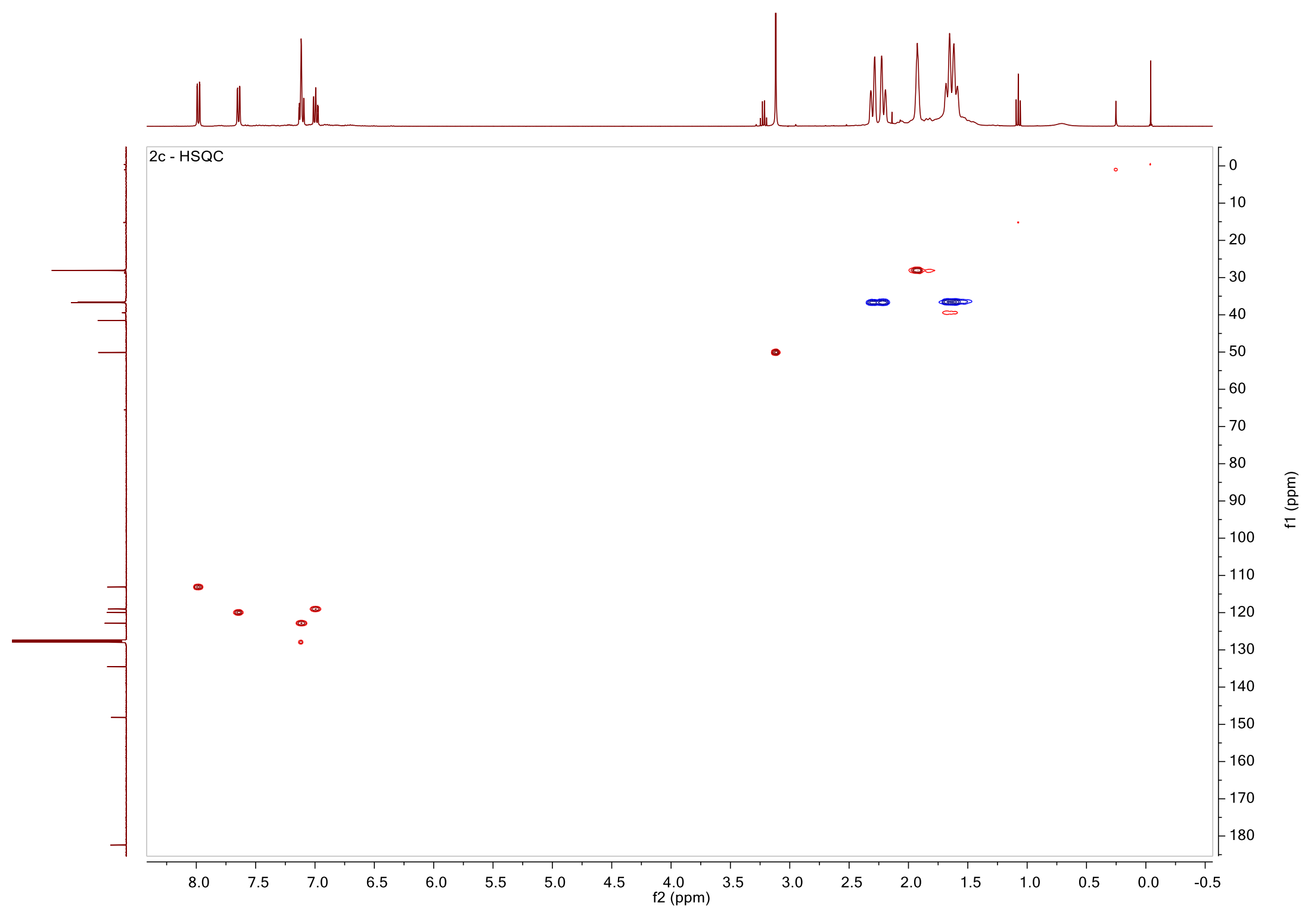


Figure $\mathbf{S} 32{ }^{1} \mathrm{H}-\mathrm{NMR}$ spectrum of $2 \mathbf{c}$ in $\mathrm{C}_{6} \mathrm{D}_{6}$ at $70{ }^{\circ} \mathrm{C}$.

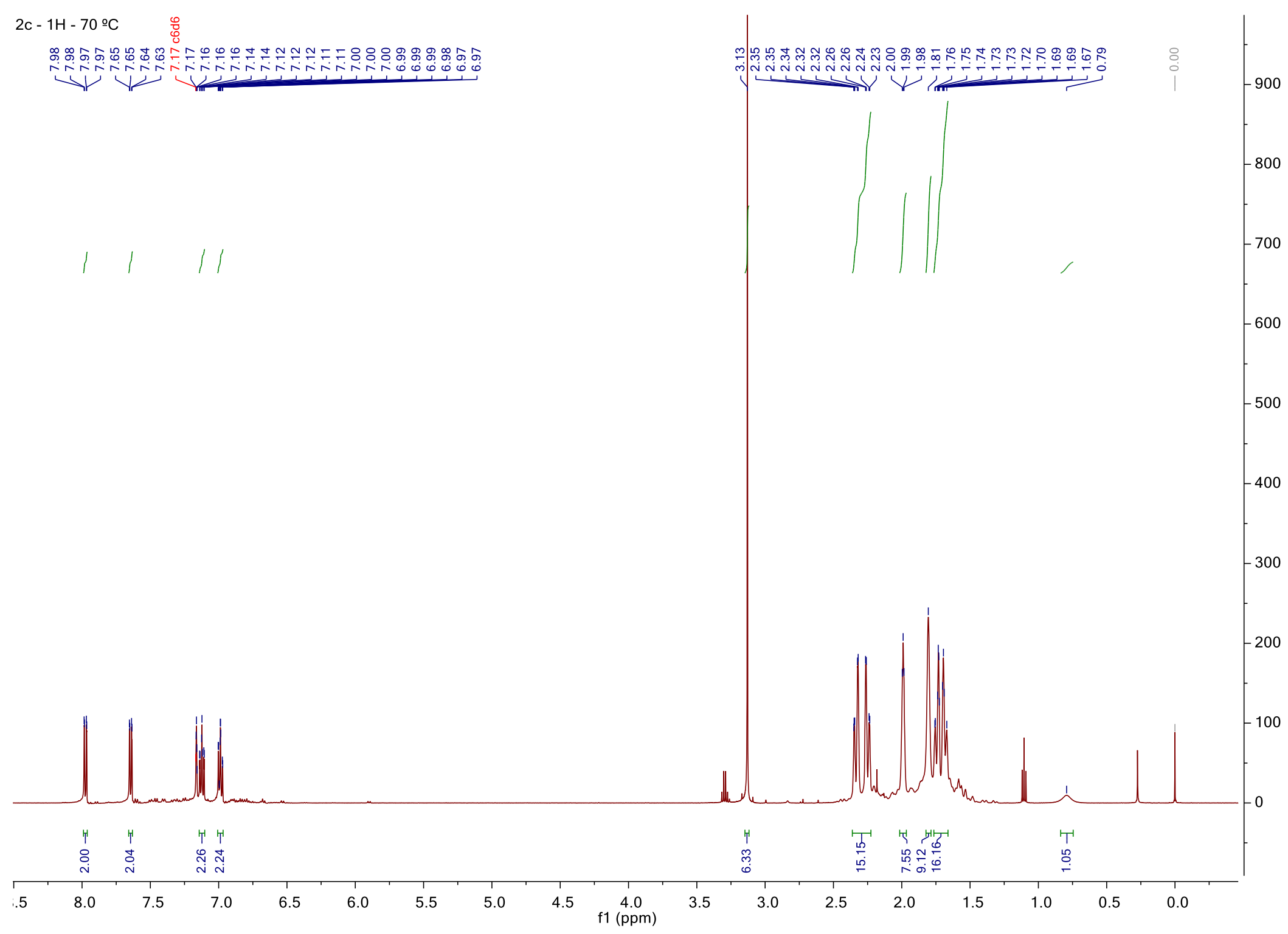


Figure $\mathbf{S} 33^{1} \mathrm{H}-\mathrm{NMR}$ spectrum of $\mathbf{2 d}$ in $\mathrm{C}_{6} \mathrm{D}_{6}$ at room temperature.

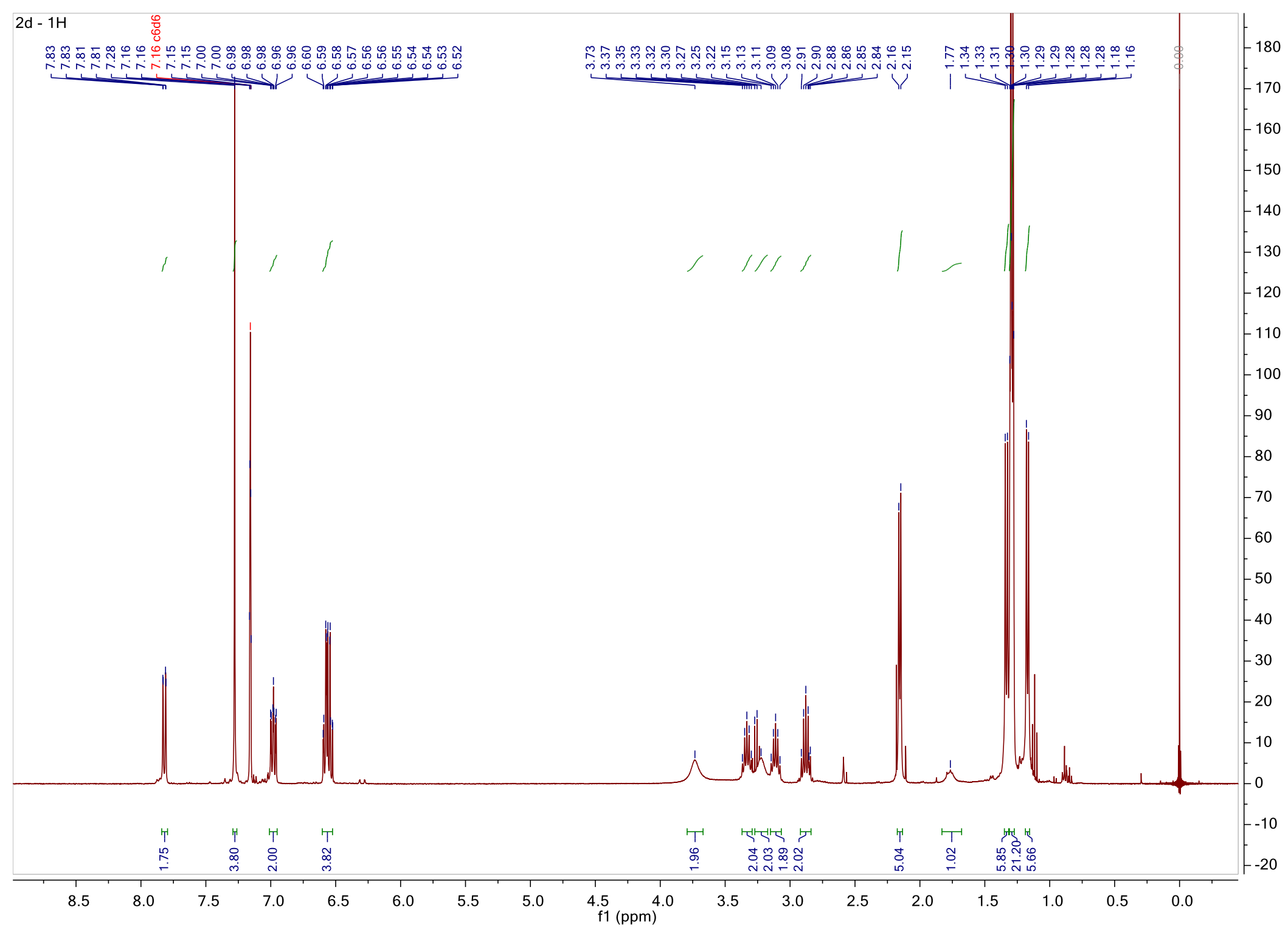


Figure $\mathbf{S} 3{ }^{13} \mathrm{C}$-NMR spectrum of $\mathbf{2} \mathbf{d}$ in $\mathrm{C}_{6} \mathrm{D}_{6}$ at room temperature.

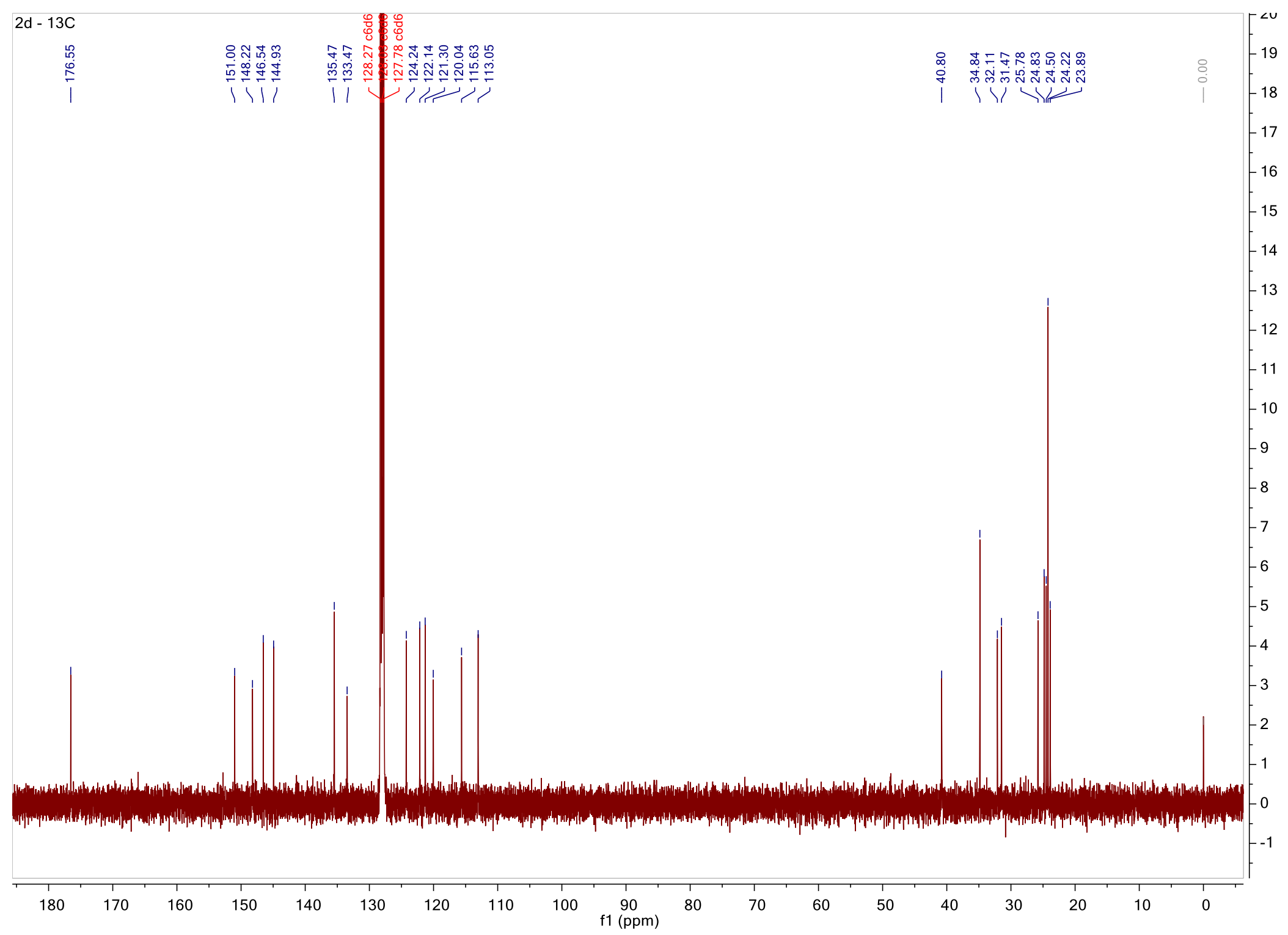


Figure S 35 HSQC-NMR spectrum of $\mathbf{2 d}$ in $\mathrm{C}_{6} \mathrm{D}_{6}$ at room temperature.

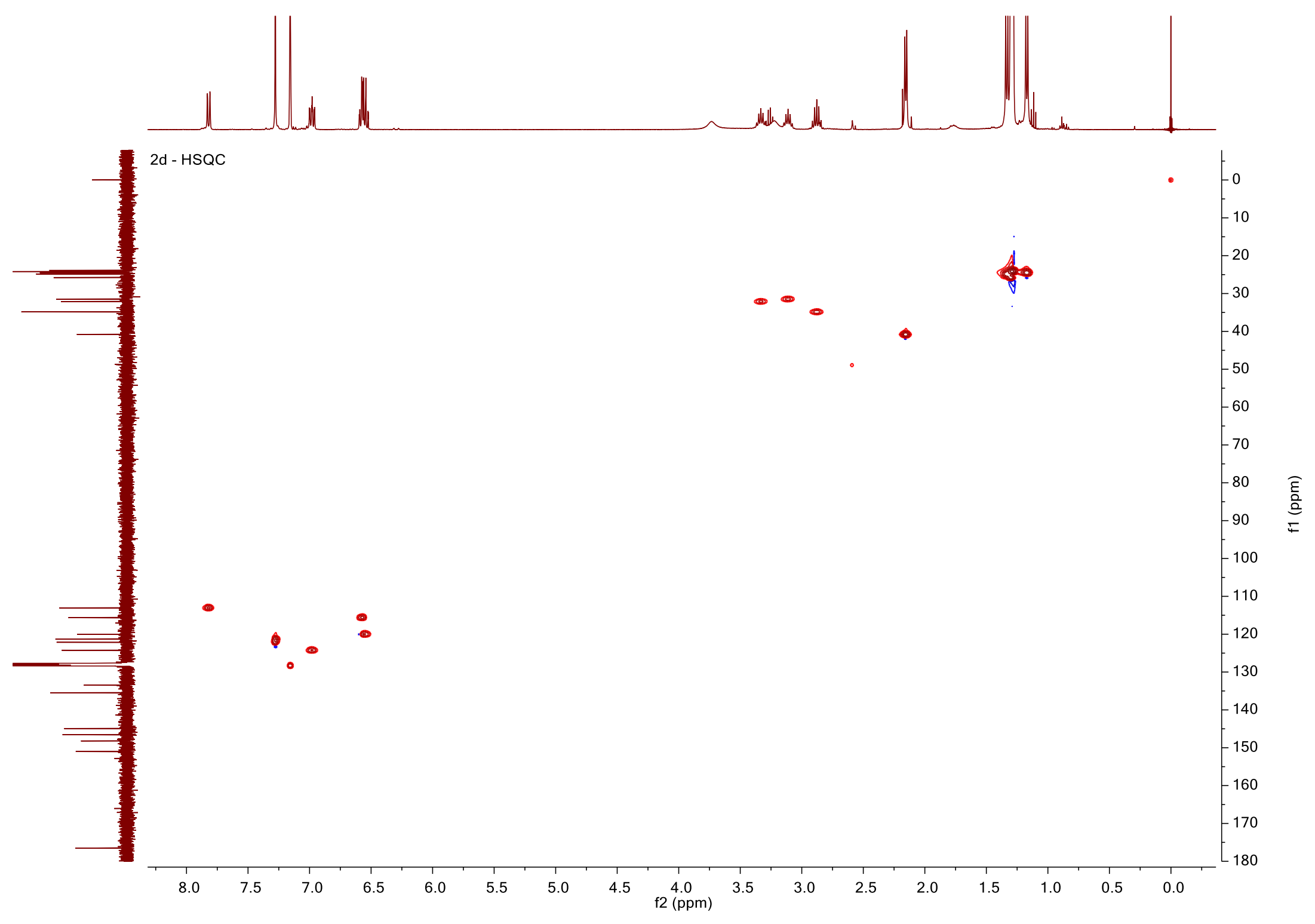


Figure $\mathbf{S} 36$ COSY-NMR spectrum of $\mathbf{2} \mathbf{d}$ in $\mathrm{C}_{6} \mathrm{D}_{6}$ at room temperature.
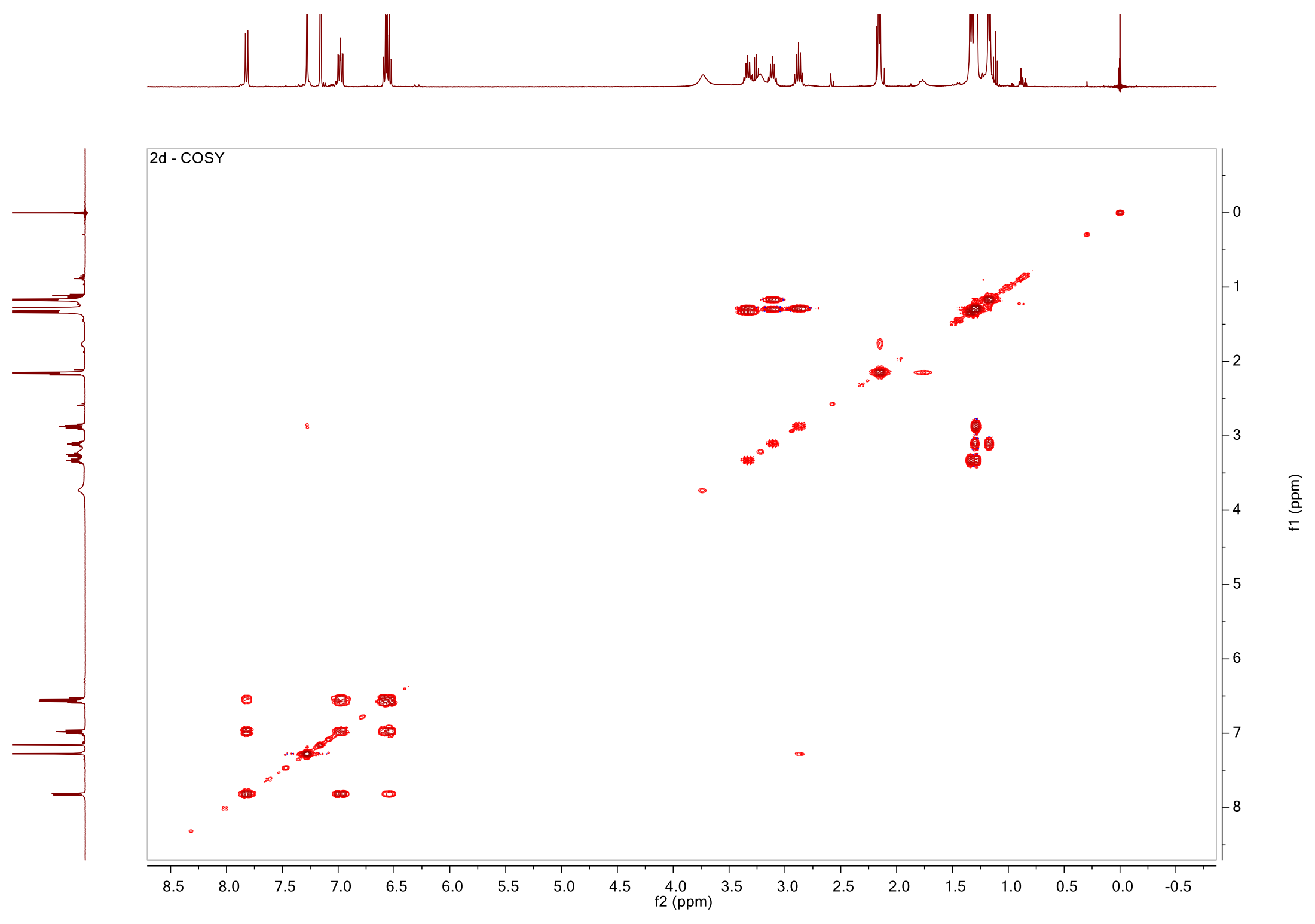
Figure S $37{ }^{1} \mathrm{H}-\mathrm{NMR}$ spectrum of $\mathbf{2 d}$ in $\mathrm{C}_{6} \mathrm{D}_{6}$ at $70{ }^{\circ} \mathrm{C}$.

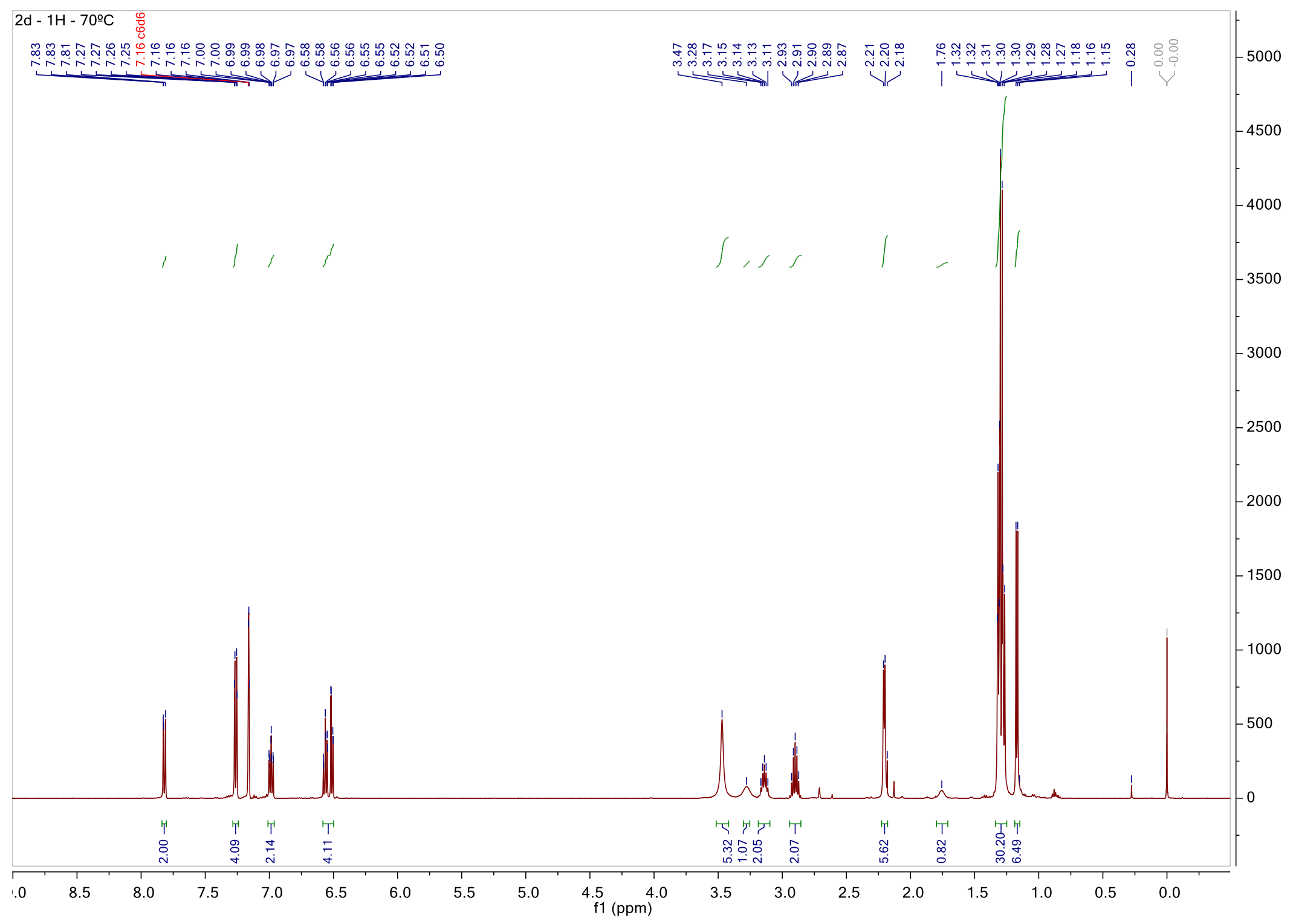


Figure $\mathbf{S} 38$ NOESY-NMR spectrum of $\mathbf{2} \mathbf{d}$ in $\mathrm{C}_{6} \mathrm{D}_{6}$ at room temperature.

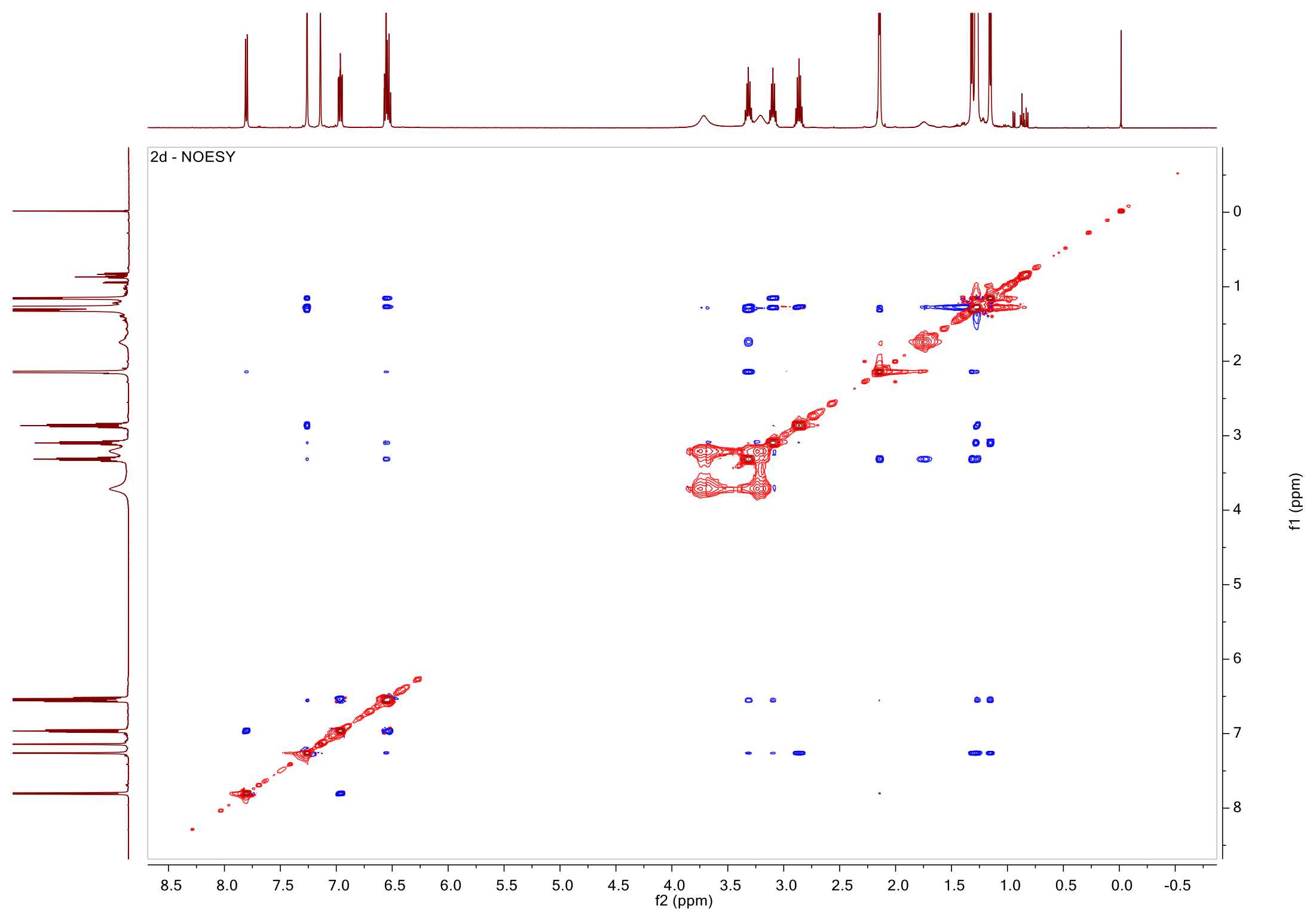


Figure S $39{ }^{1} \mathrm{H}-\mathrm{NMR}$ spectrum of $\mathbf{3 d}$ in $\mathrm{C}_{6} \mathrm{D}_{6}$ at room temperature.

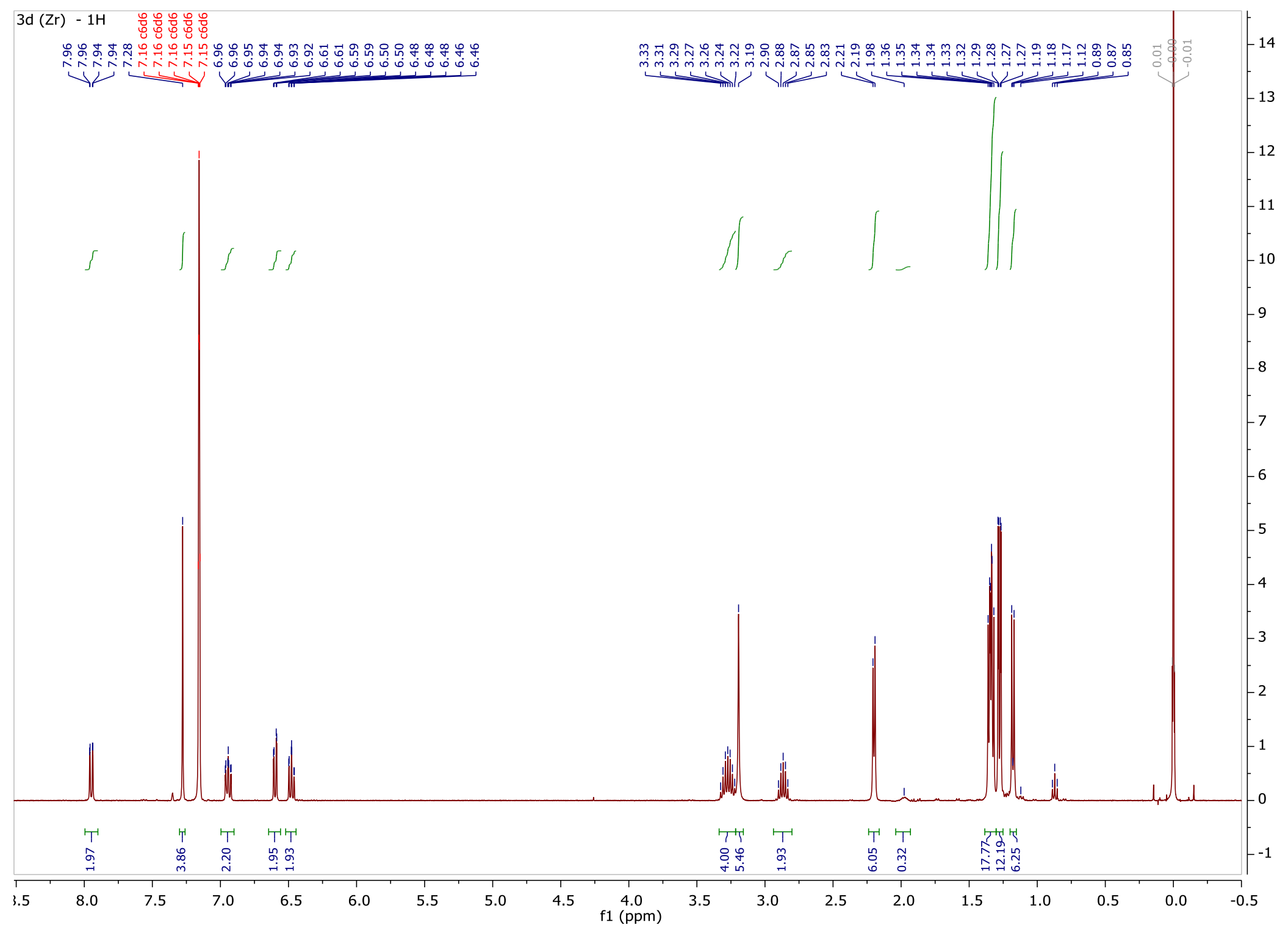


Figure $\mathbf{S} 40^{13} \mathrm{C}$-NMR spectrum of $\mathbf{3 d}$ in $\mathrm{C}_{6} \mathrm{D}_{6}$ at room temperature.

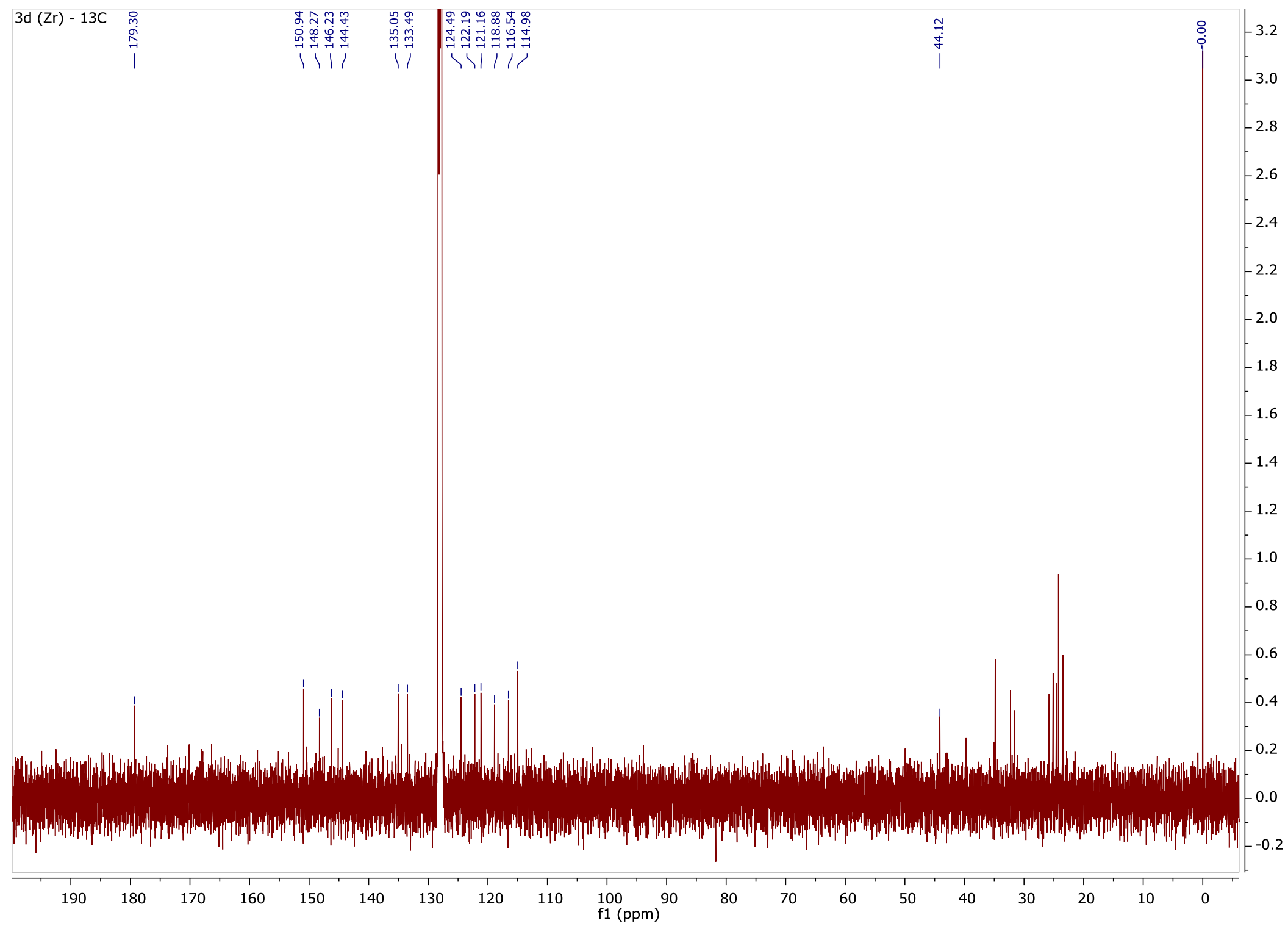


Figure S 41 HSQC-NMR spectrum of $\mathbf{3 d}$ in $\mathrm{C}_{6} \mathrm{D}_{6}$ at room temperature.

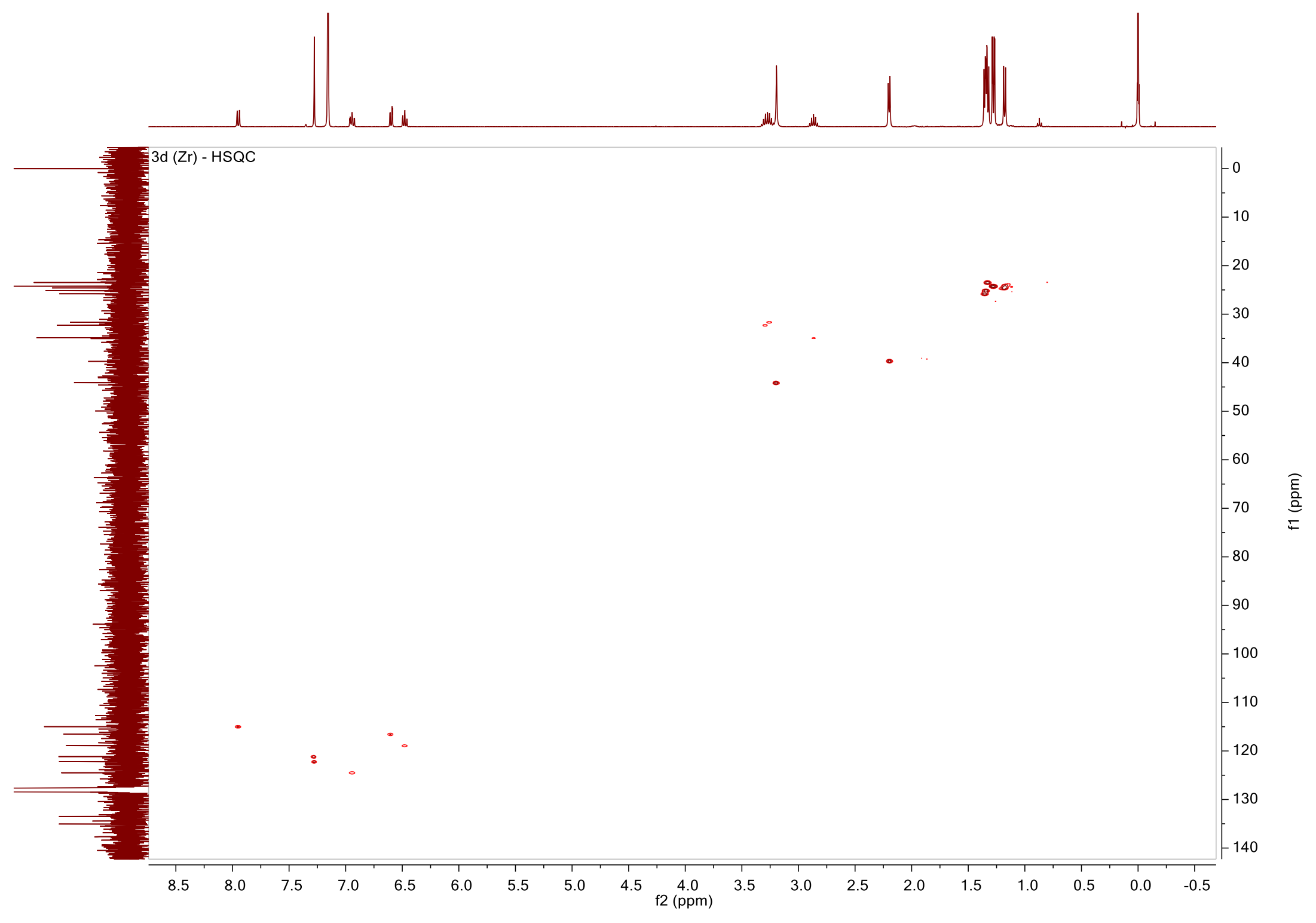


Figure S $42{ }^{1} \mathrm{H}-\mathrm{NMR}$ spectrum of $\mathbf{4 d}$ in $\mathrm{C}_{6} \mathrm{D}_{6}$ at room temperature.

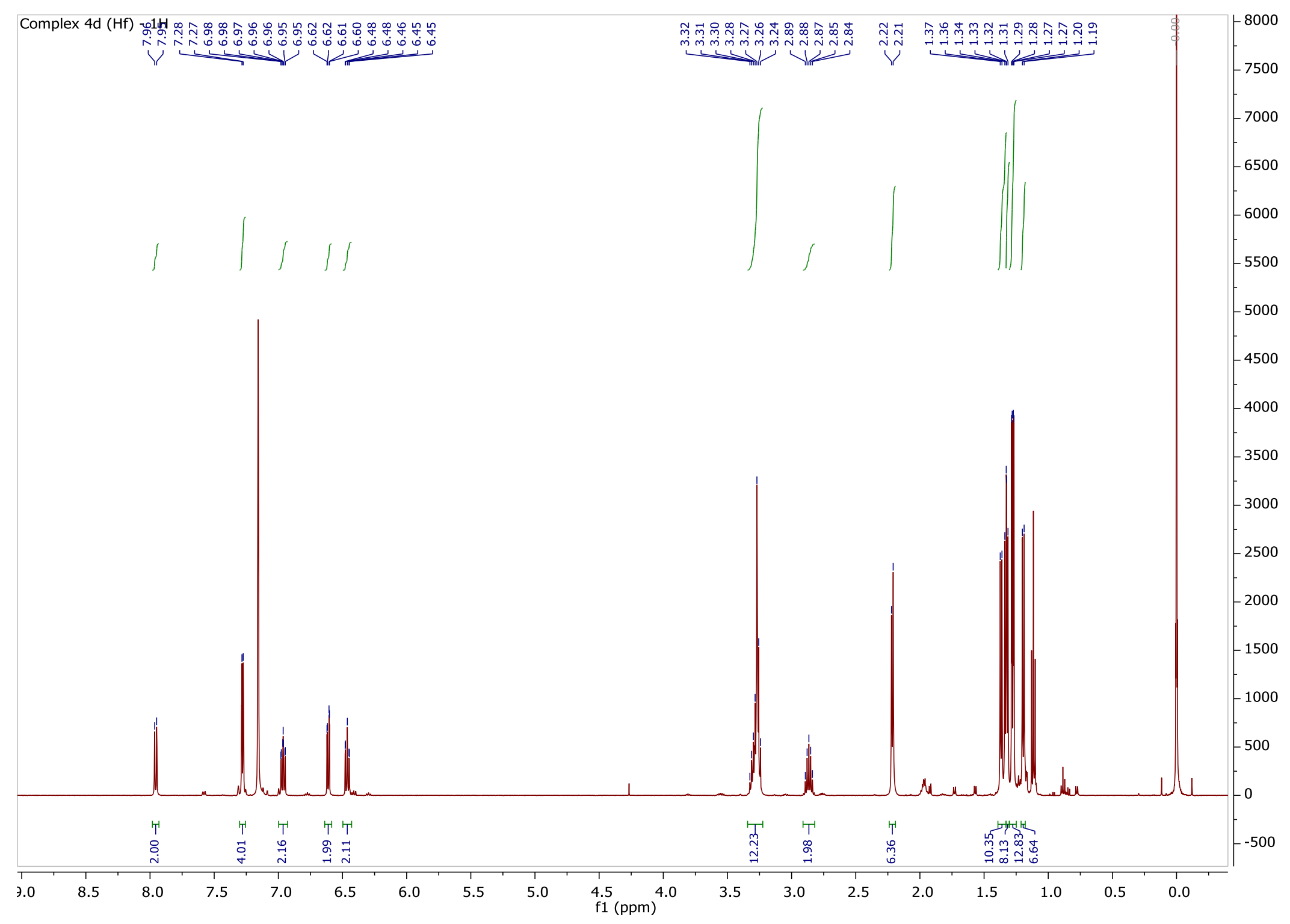


Figure $\mathbf{S} 43^{13} \mathrm{C}$-NMR spectrum of $\mathbf{4 d}$ in $\mathrm{C}_{6} \mathrm{D}_{6}$ at room temperature.

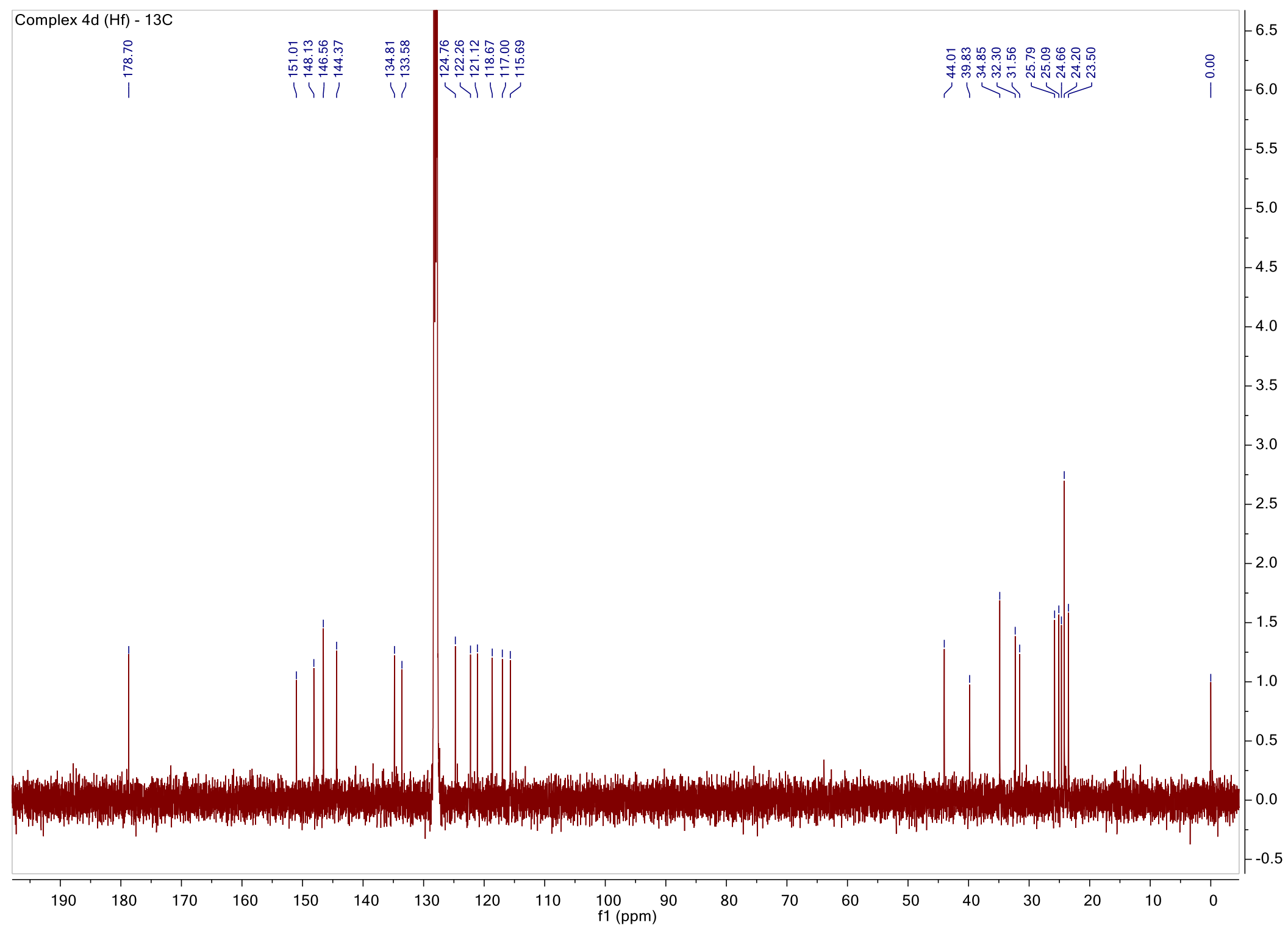


Figure S 44 HSQC-NMR spectrum of $\mathbf{4 d}$ in $\mathrm{C}_{6} \mathrm{D}_{6}$ at room temperature.

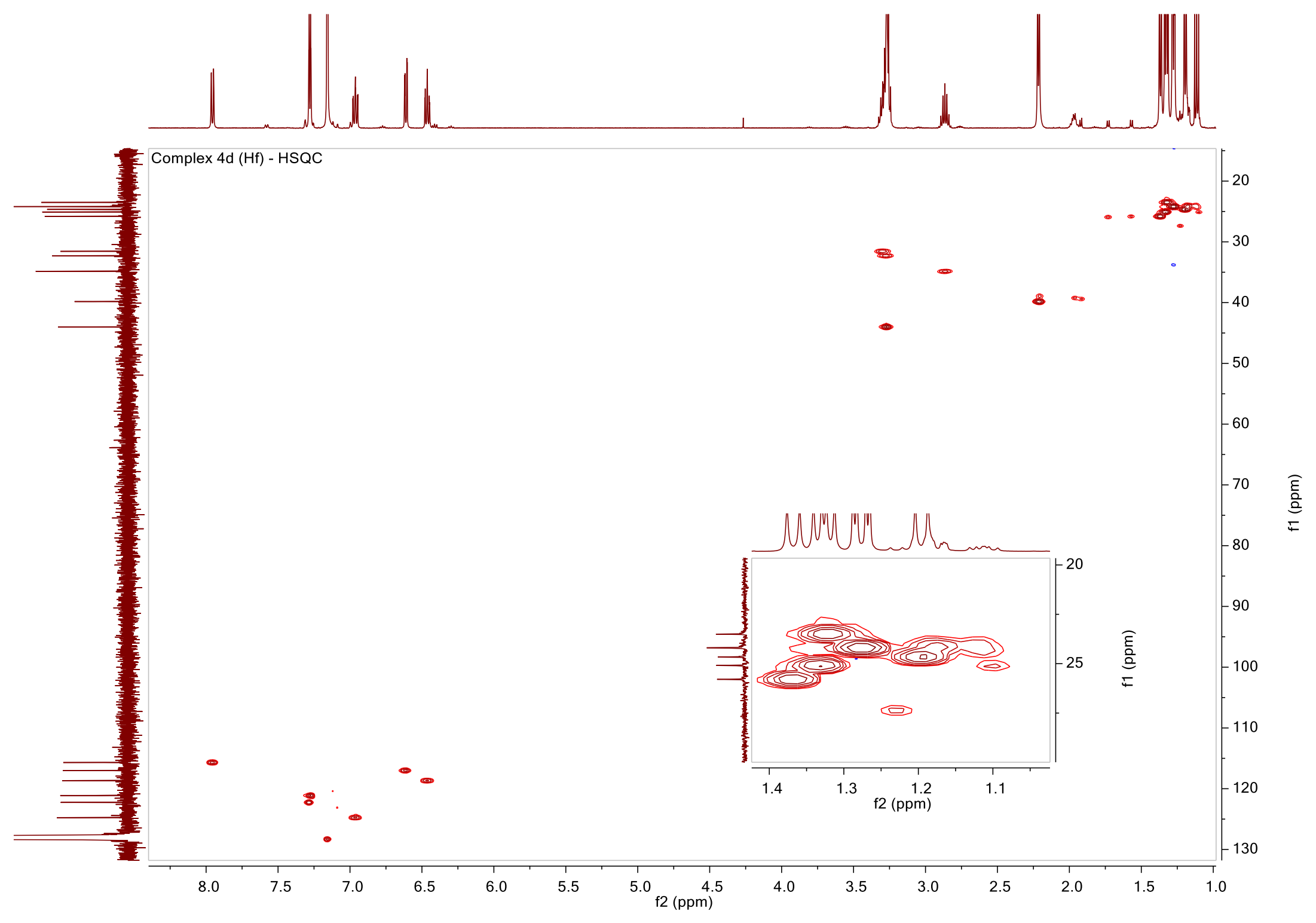


Figure S 45 COSY-NMR spectrum of $\mathbf{4 d}$ in $\mathrm{C}_{6} \mathrm{D}_{6}$ at room temperature.

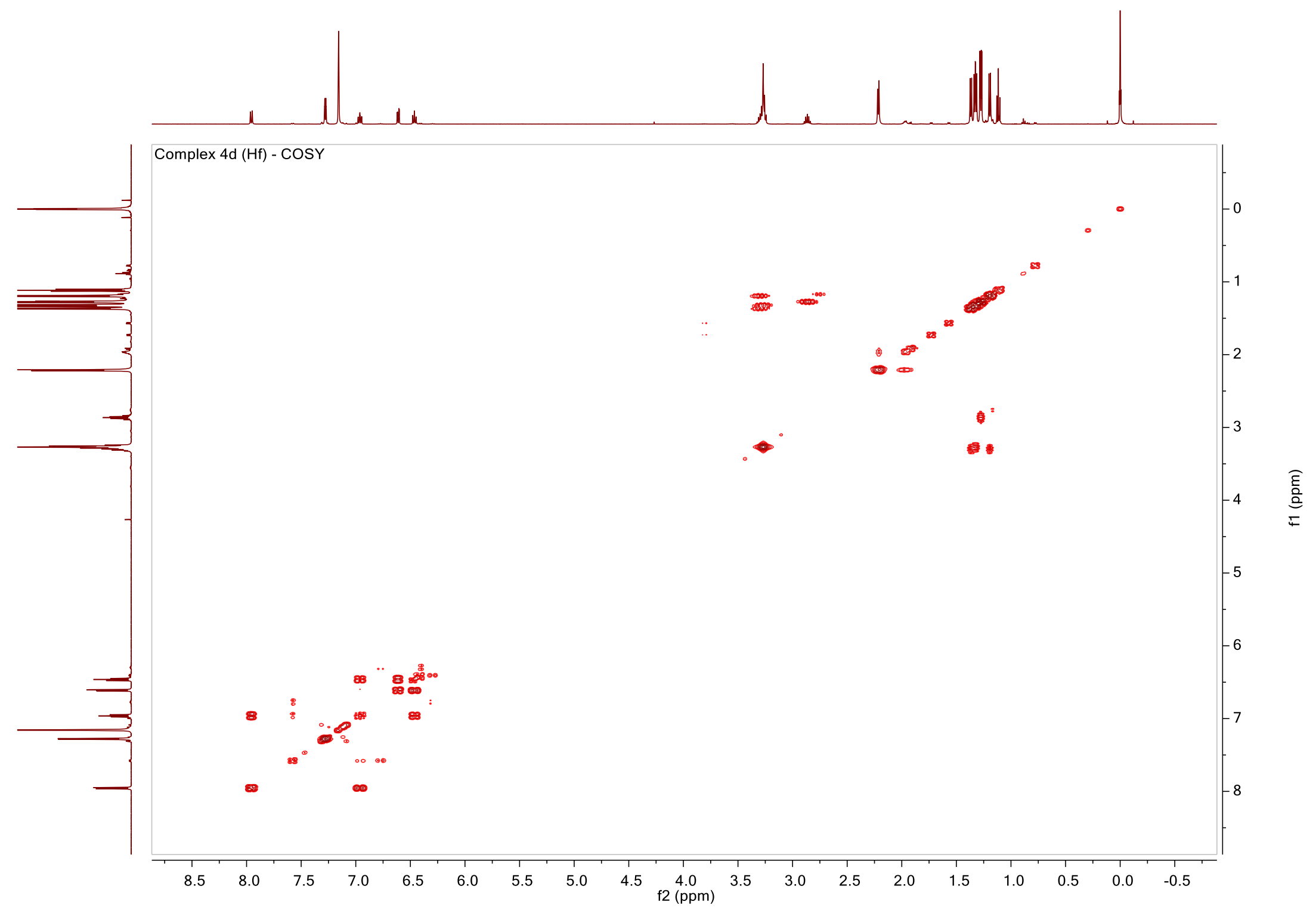


Figure S 46 NOESY-NMR spectrum of $\mathbf{4 d}$ in $\mathrm{C}_{6} \mathrm{D}_{6}$ at room temperature.

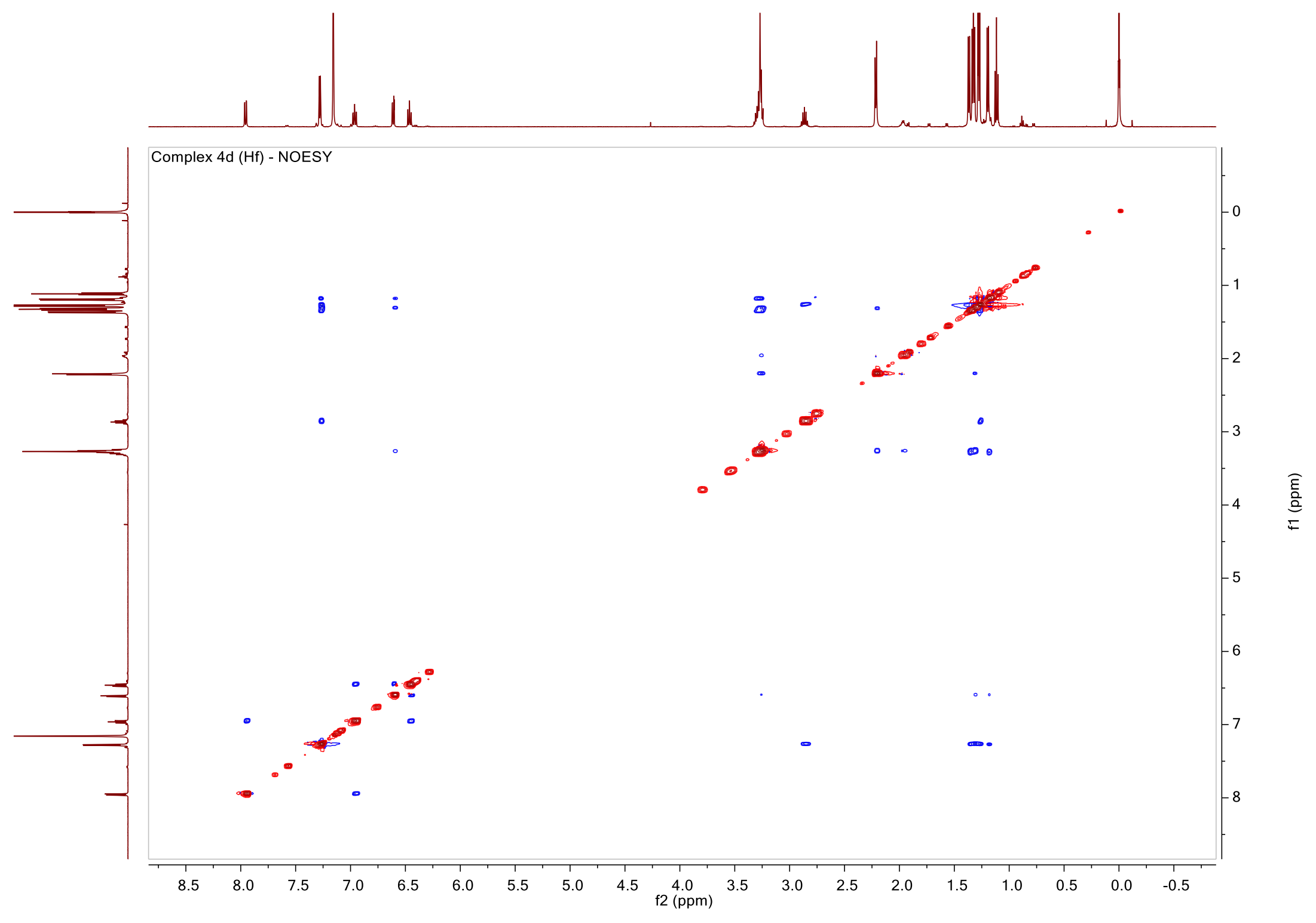


Figure $\mathbf{S} 47^{1} \mathrm{H}-\mathrm{NMR}$ spectrum of $\mathbf{5 d}$ in $\mathrm{C}_{6} \mathrm{D}_{6}$ at room temperature.

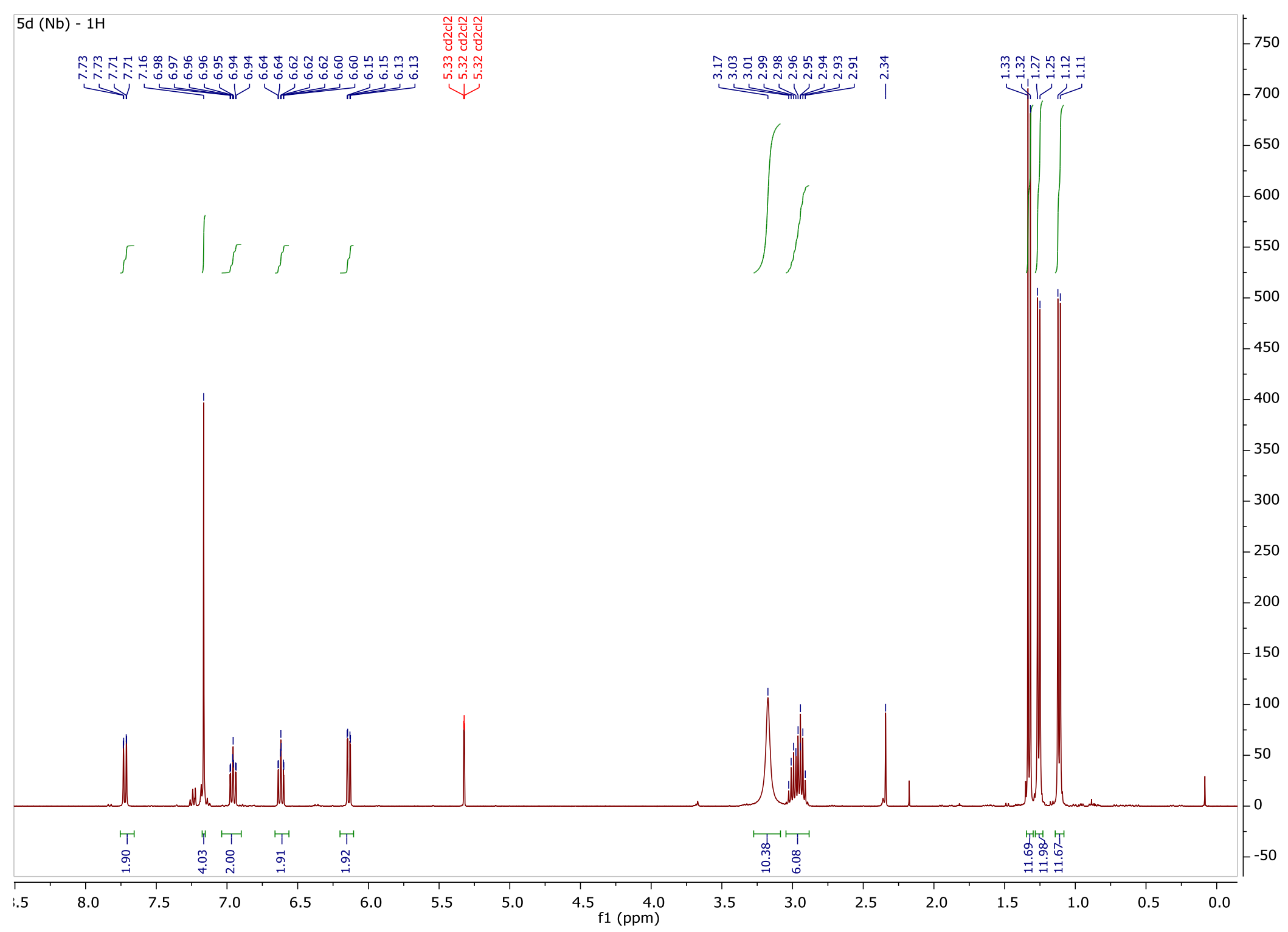


Figure $\mathbf{S} 48{ }^{13} \mathrm{C}-\mathrm{NMR}$ spectrum of $\mathbf{5} \mathbf{d}$ in $\mathrm{C}_{6} \mathrm{D}_{6}$ at room temperature.

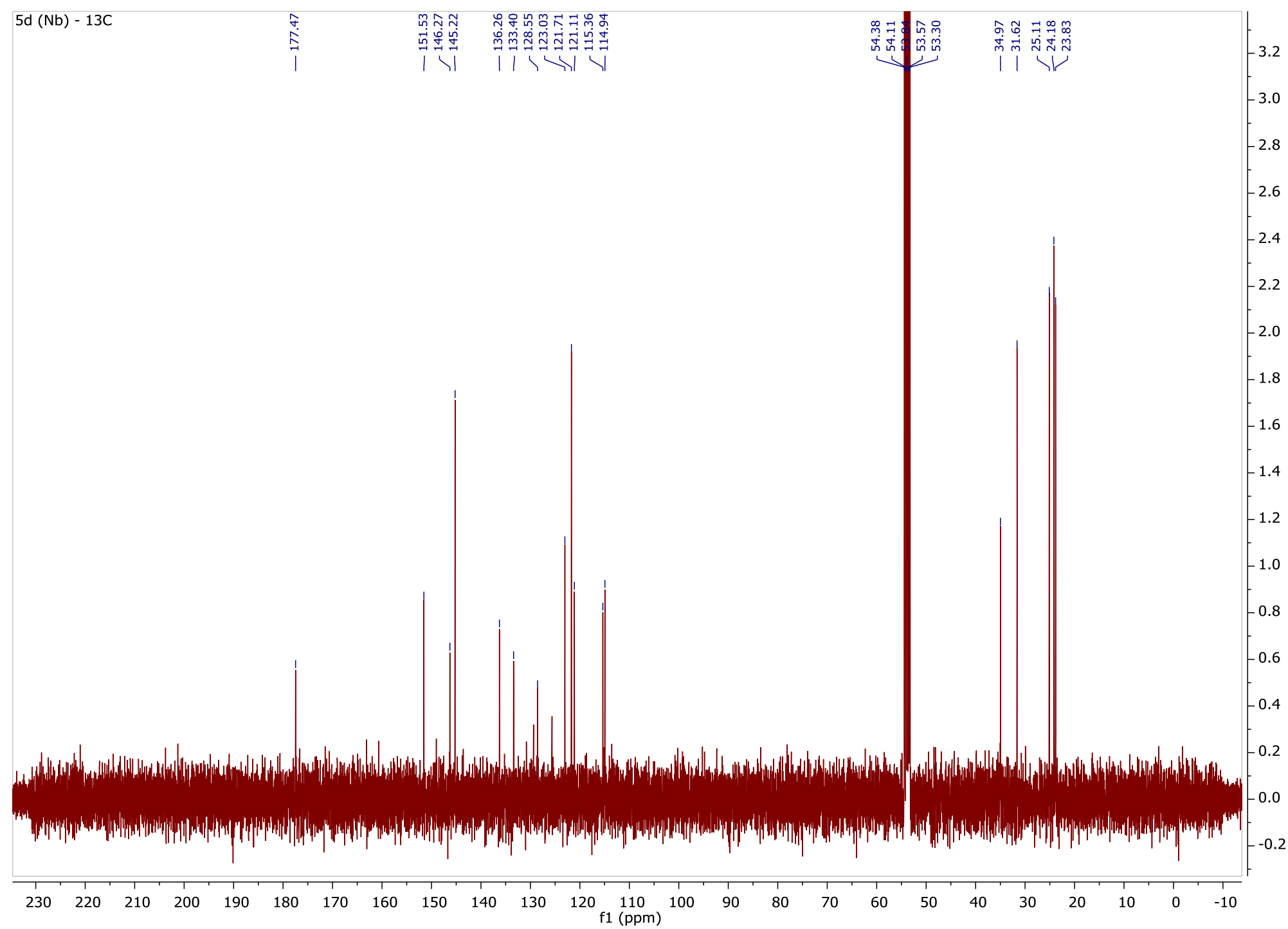


Figure $\mathbf{S} 49 \mathrm{HSQC}-\mathrm{NMR}$ spectrum of $\mathbf{5} \mathbf{d}$ in $\mathrm{C}_{6} \mathrm{D}_{6}$ at room temperature.

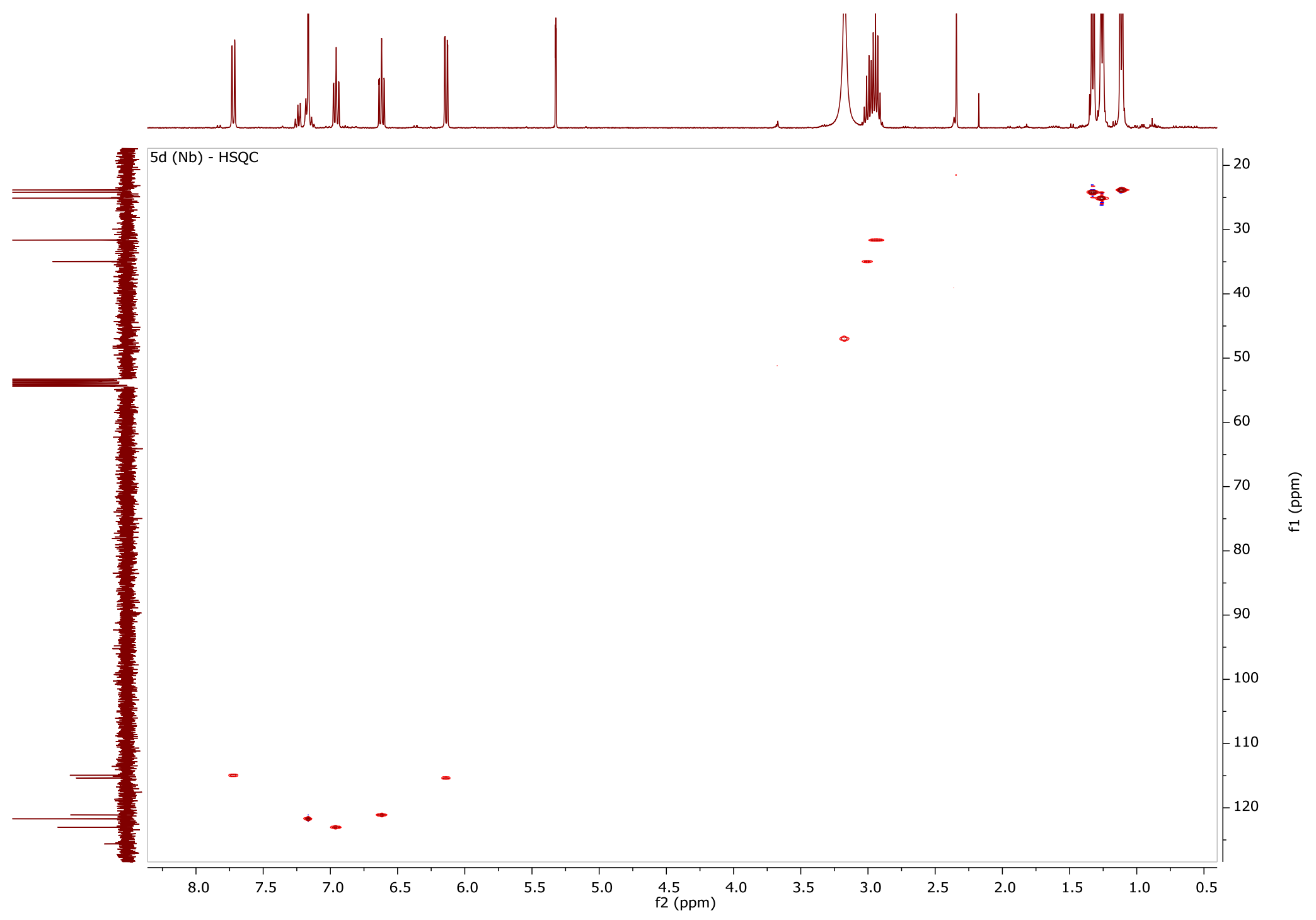


Figure $\mathbf{S} 50$ COSY-NMR spectrum of $\mathbf{5 d}$ in $\mathrm{C}_{6} \mathrm{D}_{6}$ at room temperature.

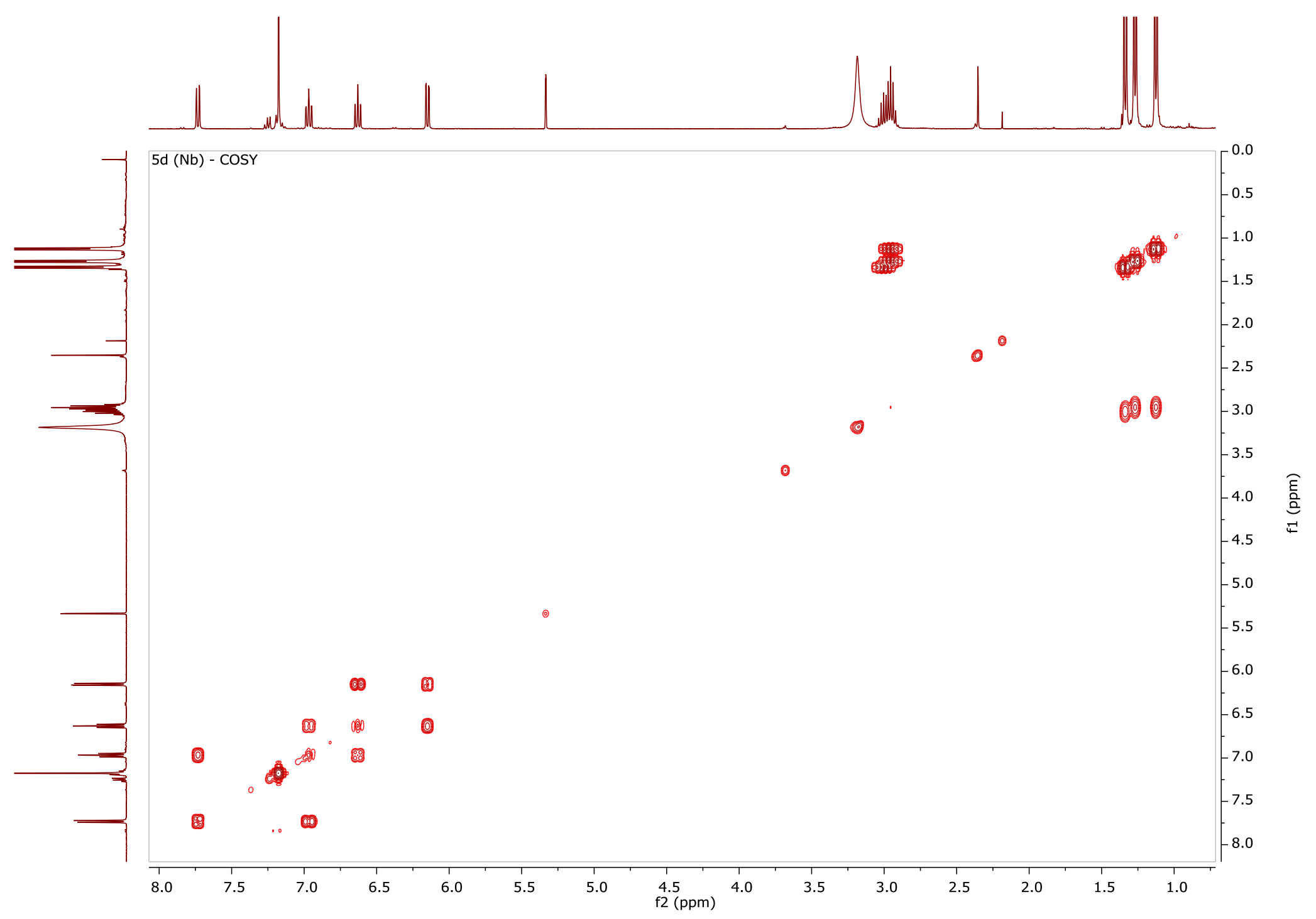


Figure $\mathbf{S} 51^{1} \mathrm{H}-\mathrm{NMR}$ spectrum of $\mathbf{6 d}$ in $\mathrm{C}_{6} \mathrm{D}_{6}$ at room temperature.

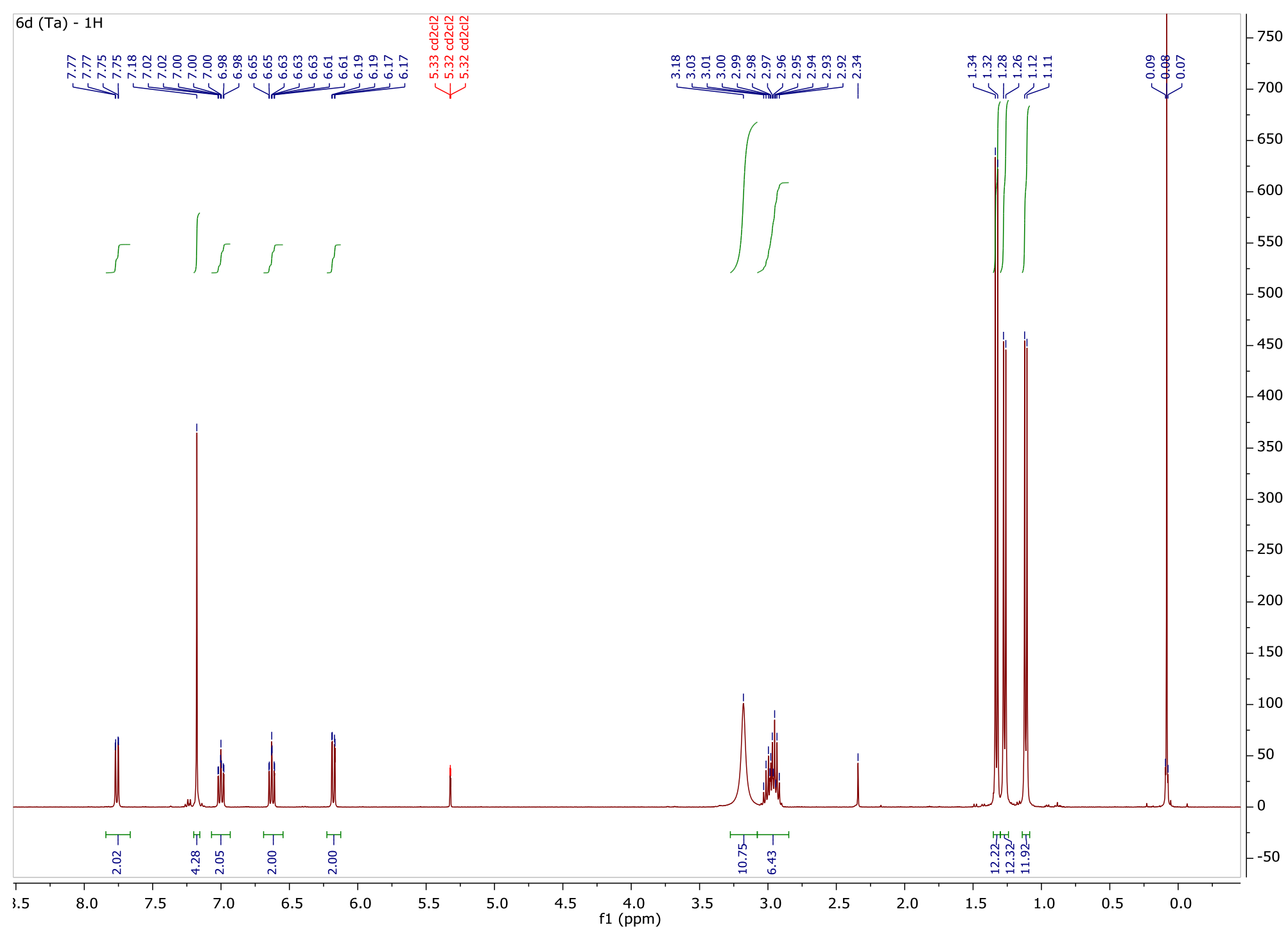


Figure $\mathbf{S} 52{ }^{13} \mathrm{C}-\mathrm{NMR}$ spectrum of $\mathbf{6} \mathbf{d}$ in $\mathrm{C}_{6} \mathrm{D}_{6}$ at room temperature.

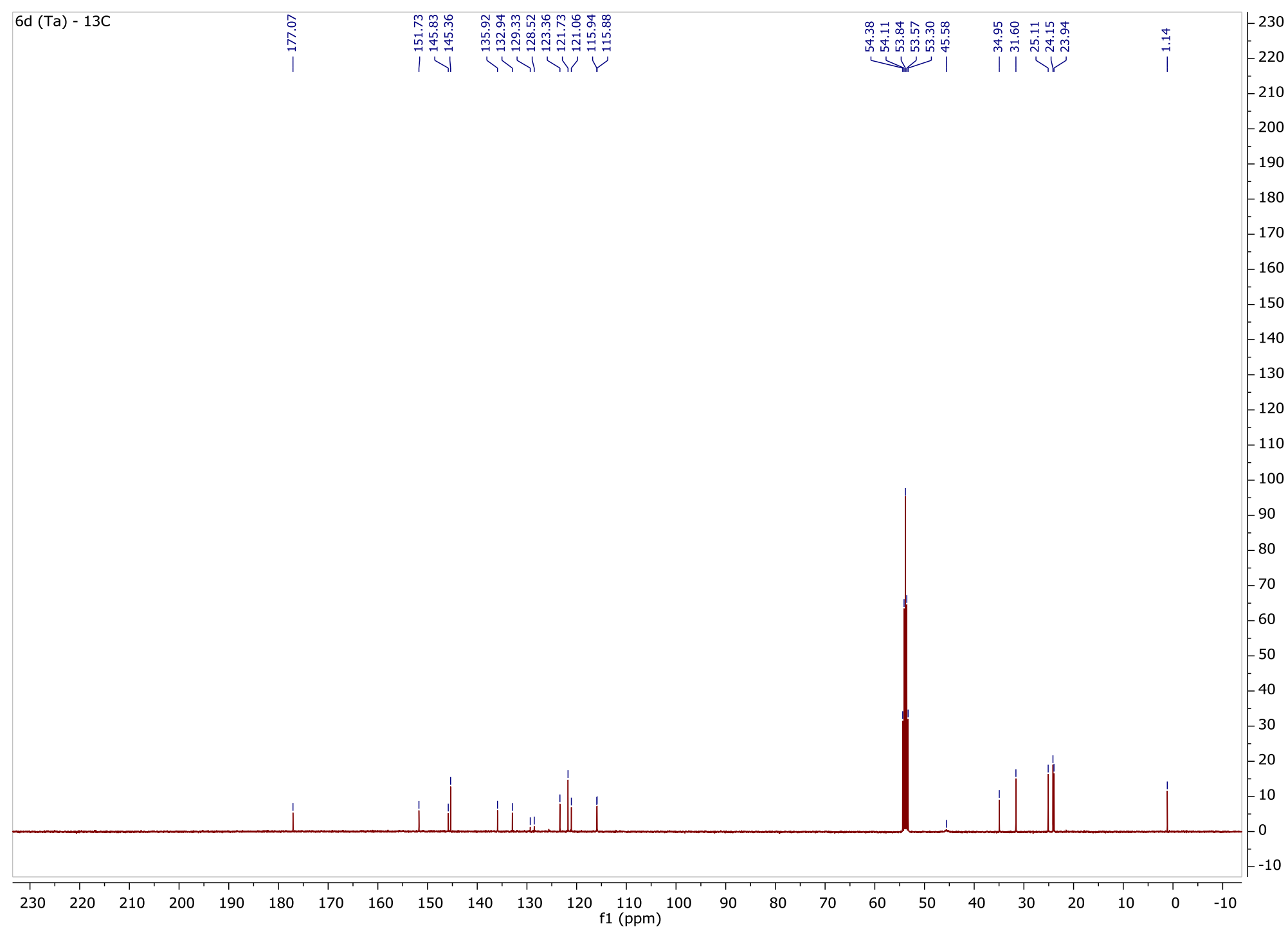


Figure $\mathbf{S} 53$ HSQC-NMR spectrum of $\mathbf{6 d}$ in $\mathrm{C}_{6} \mathrm{D}_{6}$ at room temperature.

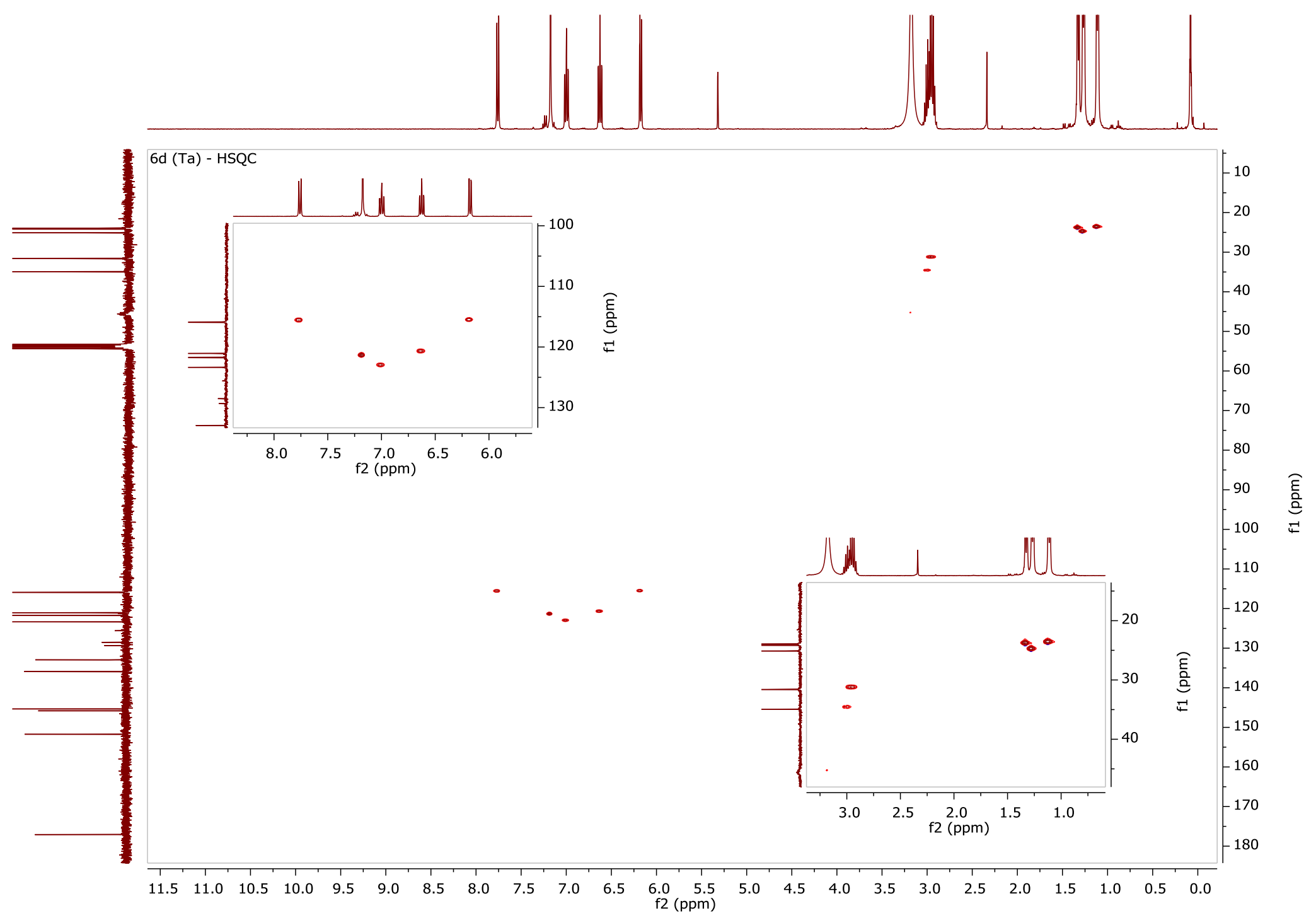

Figure S 54 COSY-NMR spectrum of $\mathbf{6} \mathbf{d}$ in $\mathrm{C}_{6} \mathrm{D}_{6}$ at room temperature. 


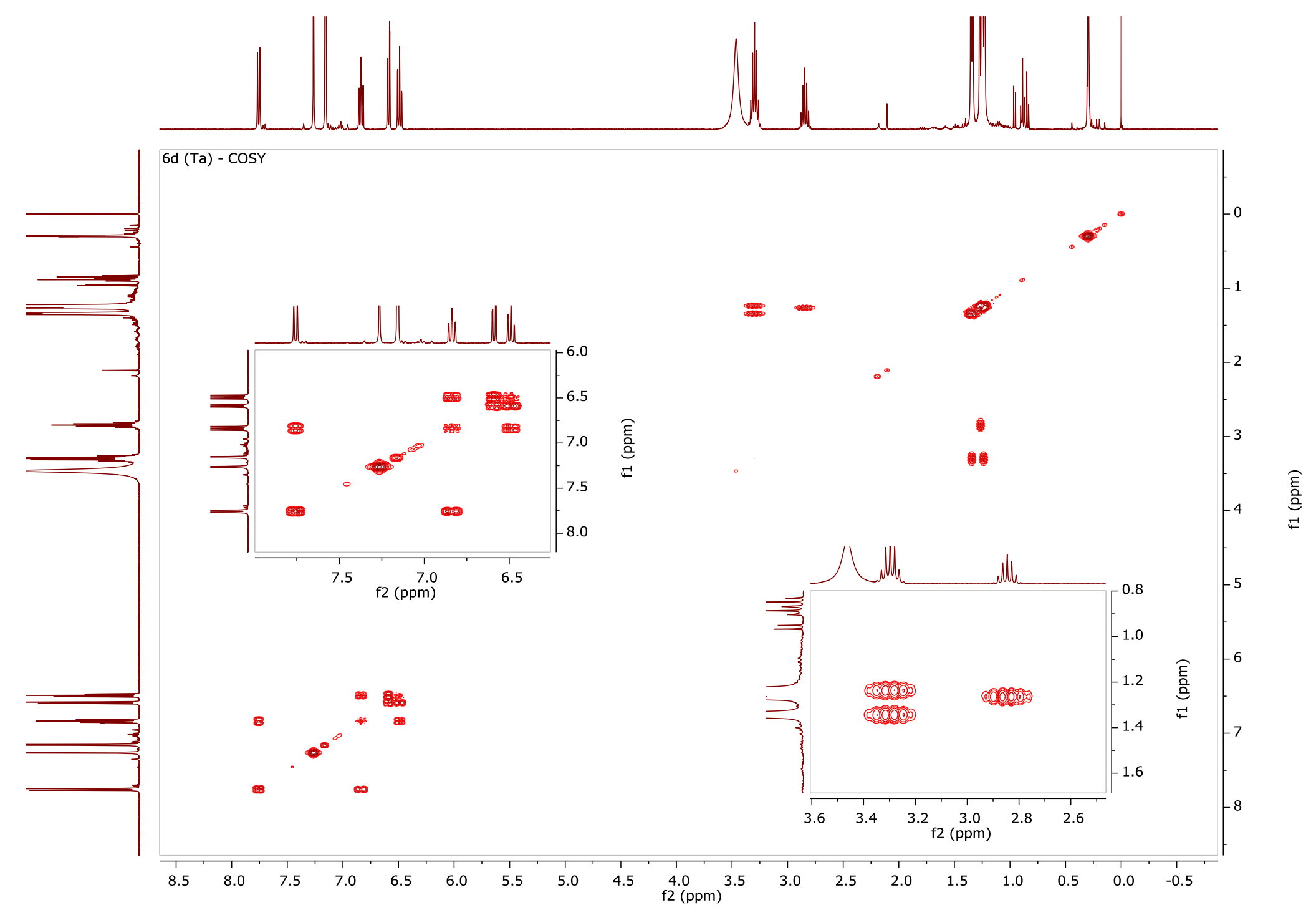

